\title{
Disentangling the Circumnuclear Environs of Centaurus A. III. An Inner Molecular Ring, Nuclear Shocks, and the $\mathrm{CO}$ to Warm $\mathrm{H}_{2}$ Interface
}

\author{
D. Espada ${ }^{1,2,3}$, S. Matsushita ${ }^{4}$, R. E. Miura ${ }^{1}$, F. P. Israel ${ }^{5}$, N. Neumayer ${ }^{6}$, S. Martin ${ }^{3,7}$, C. Henkel ${ }^{8,9}$, T. Izumi ${ }^{10}$, D. Iono ${ }^{1,2}$, \\ S. Aalto ${ }^{11}$, J. Ott ${ }^{12}$, A. B. Peck ${ }^{13}$, A. C. Quillen ${ }^{14}$, and K. Kohno ${ }^{10}$ \\ ${ }^{1}$ National Astronomical Observatory of Japan, 2-21-1 Osawa, Mitaka, Tokyo 181-8588, Japan \\ ${ }^{2}$ The Graduate University for Advanced Studies (SOKENDAI), 2-21-1 Osawa, Mitaka, Tokyo, 181-0015, Japan \\ ${ }^{3}$ Joint ALMA Observatory, Alonso de Córdova, 3107, Vitacura, Santiago 763-0355, Chile \\ ${ }^{4}$ Academia Sinica, Institute of Astronomy \& Astrophysics, P.O. Box 23-141, Taipei 10617, Taiwan \\ ${ }^{5}$ Sterrewacht Leiden, Leiden University, P.O. Box 9513, 2300 RA, Leiden, The Netherlands \\ ${ }^{6}$ Max Planck Institute for Astronomy (MPIA), Königstuhl 17, D-69121 Heidelberg, Germany \\ ${ }_{7}$ European Southern Observatory, Alonso de Córdova 3107, Vitacura, Santiago, Chile \\ ${ }^{8}$ Max-Planck-Institut für Radioastronomie, Auf dem Hügel 69, D-53121, Bonn, Germany \\ ${ }^{9}$ Department of Astronomy, King Abdulaziz University, P.O. Box 80203, 21589 Jeddah, Saudi Arabia \\ ${ }^{10}$ Institute of Astronomy, School of Science, The University of Tokyo, 2-21-1 Osawa, Mitaka, Tokyo 181-0015, Japan \\ ${ }^{11}$ Department of Earth and Space Sciences, Chalmers University, Sweden \\ ${ }^{12}$ National Radio Astronomy Observatory, Socorro, NM, USA \\ ${ }^{13}$ Gemini Observatory, 670 N'Aohoku Pl, Hilo 96720-2700, Hawaii, HI, USA \\ ${ }^{14}$ Department of Physics and Astronomy, University of Rochester, Rochester, NY 14627, USA \\ Received 2017 March 24; revised 2017 June 7; accepted 2017 June 9; published 2017 July 13
}

\begin{abstract}
We present the distribution and kinematics of the molecular gas in the circumnuclear disk $(\mathrm{CND} ; 400 \mathrm{pc} \times 200 \mathrm{pc})$ of Centaurus A with resolutions of $\sim 5 \mathrm{pc}(0$ !" 3$)$ and shed light onto the mechanism feeding the active galactic nucleus (AGN) using $\mathrm{CO}(3-2), \mathrm{HCO}^{+}(4-3), \mathrm{HCN}(4-3)$, and $\mathrm{CO}(6-5)$ observations obtained with ALMA. Multiple filaments or streamers of tens to a hundred parsec scale exist within the CND, which form a ring-like structure with an unprojected diameter of $9^{\prime \prime} \times 6^{\prime \prime}(162 \mathrm{pc} \times 108 \mathrm{pc})$ and a position angle P.A. $\simeq 155^{\circ}$. Inside the nuclear ring, there are two leading and straight filamentary structures with lengths of about 30-60 pc at P.A. $\simeq 120^{\circ}$ on opposite sides of the AGN, with a rotational symmetry of $180^{\circ}$ and steeper position-velocity diagrams, which are interpreted as nuclear shocks due to non-circular motions. Along the filaments, and unlike other nearby AGNs, several dense molecular clumps present low $\mathrm{HCN} / \mathrm{HCO}^{+}(4-3)$ ratios $(\lesssim 0.5)$. The filaments abruptly end in the probed transitions at $r \simeq 20$ pc from the AGN, but previous near-IR $\mathrm{H}_{2}(J=1-0) \mathrm{S}(1)$ maps show that they continue in an even warmer gas phase $(T \sim 1000 \mathrm{~K})$, winding up in the form of nuclear spirals, and forming an inner ring structure with another set of symmetric filaments along the $\mathrm{N}-\mathrm{S}$ direction and within $r \simeq 10 \mathrm{pc}$. The molecular gas is governed primarily by non-circular motions, being the successive shock fronts at different scales where loss of angular momentum occurs, a mechanism that may feed efficiently powerful radio galaxies down to parsec scales.
\end{abstract}

Key words: galaxies: elliptical and lenticular, cD - galaxies: individual (NGC 5128) - galaxies: structure ISM: molecules

\section{Introduction}

Active galactic nuclei (AGNs) are thought to be powered by accretion onto supermassive black holes (SMBHs) and their luminosities require large mass accretion rates. However, a crucial outstanding problem is to identify the mechanisms that drive gas from external regions toward the nuclei of these galaxies, removing its angular momentum in order to trigger nuclear activity and feed the SMBHs that reside there. On the other hand, AGNs will have an effect on the surrounding molecular gas, from positive feedback, such as gas compressed by jets or winds, to negative feedback, where outflows also drive gas away from the nuclear region (for reviews see Fabian 2012; King \& Pounds 2015).

In powerful radio galaxies, the properties of the circumnuclear gas from hundreds of parsec scale down to the accretion disk are thus far poorly understood. Their luminosities, which may exceed $10^{46} \mathrm{erg} \mathrm{s}^{-1}$, require mass accretion rates of $>1 M_{\odot} \mathrm{yr}^{-1}$. Radio galaxies are radio-loud active galaxies, usually of elliptical type. Their large-scale synchrotron jets are presumably powered by the accretion of gas onto
SMBHs, which are fueled by reservoirs of neutral and ionized gas in the host galaxy. Some of these radio galaxies possess dust lanes containing large amounts of material comprising different gas phases of the interstellar medium (e.g., Allen et al. 2002). Powerful radio sources are rare in the local universe, and thus the lack of high-resolution observations have so far inhibited studies of the properties of the molecular gas in their nuclear regions.

Shlosman et al. (1989) proposed that funneling gas to the centers of galaxies may occur from large to small scales, due to successive dynamical instabilities, also known as the bars within bars mechanism, in which a primary bar may drive the gas inwards, forming a circumnuclear disk which then becomes unstable again to form a decoupled nuclear bar. Although many observational and numerical studies of spiral galaxies have been carried out (e.g., Pfenniger \& Norman 1990; Friedli \& Martinet 1993; Jungwiert et al. 1997; Englmaier \& Shlosman 2004), a similar scenario may also be present in elliptical galaxies, where material accreted from external sources is not supported rotationally. This gas would go into Keplerian orbits close to the center and may form a gaseous 


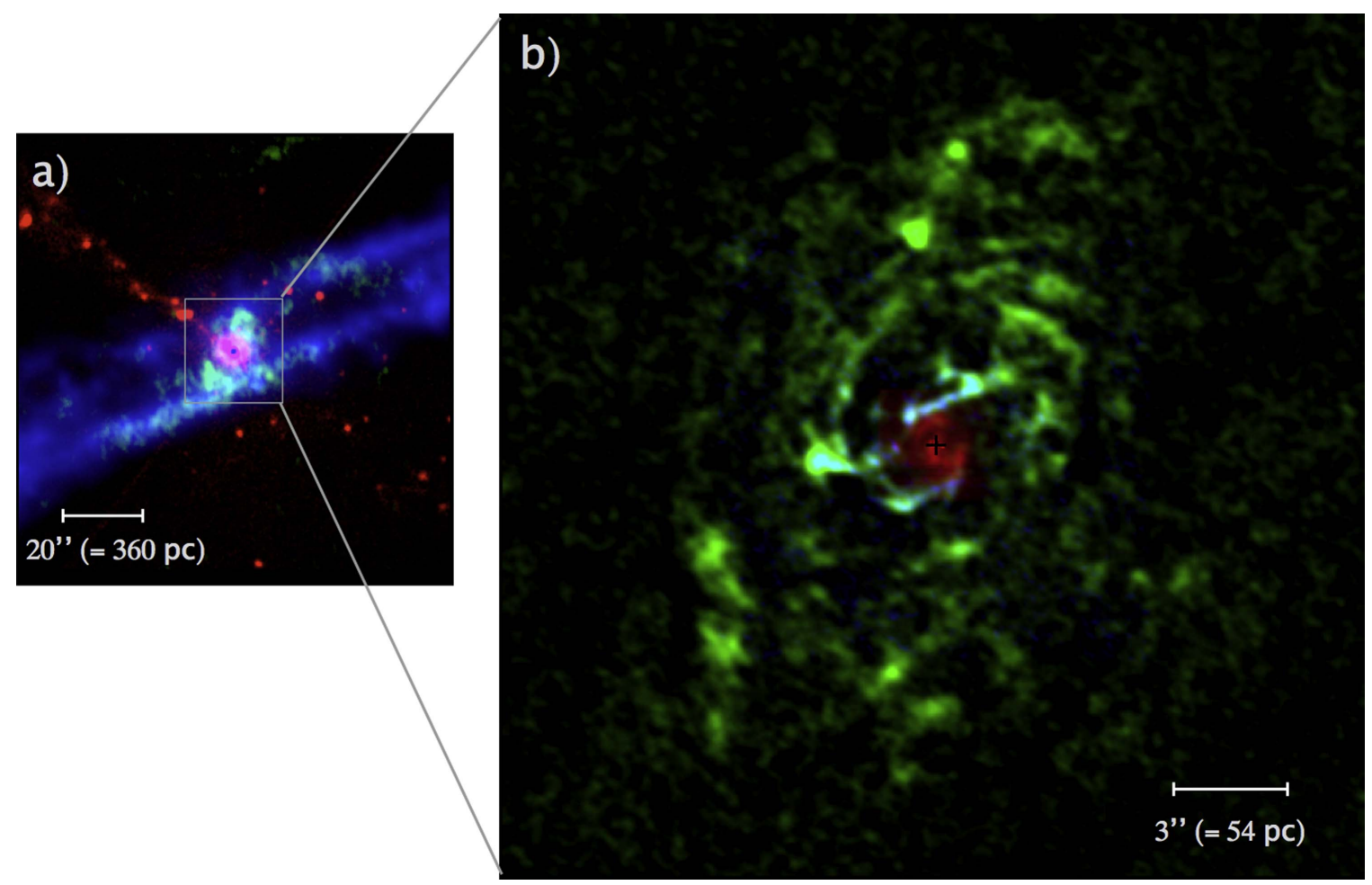

Figure 1. Kiloparsec- to parsec-scale view of the molecular disk of Cen A (NGC 5128). (a) Integrated $\mathrm{CO}(2-1)$ emission map (green) observed using the Submillimeter Array (SMA; Espada et al. 2009). The (white) rectangle encompasses the molecular gas in the CND $(r<200 \mathrm{pc})$ in the form of a disk/torus just perpendicular to the X-ray/radio jet (red, Chandra/ACIS-I; Kraft et al. 2002) and represents the area covered by the $\mathrm{CO}(3-2)$ observations as shown in panel (b). A more extended molecular gas component in the form of spiral arms (Espada et al. 2012) is seen to be coextensive with a parallelogram structure previously observed in dust emission along P.A. $=120^{\circ}$ (blue, $8 \mu \mathrm{m}$ Spitzer/IRAC; Quillen et al. 2006). (b) Composite image of the CND of Cen A including the ALMA CO(3-2) (green) and $\mathrm{CO}(6-5)$ (blue) integrated intensity maps, as presented in this paper, as well as VLT/SINFONI $\mathrm{H}_{2}(1-0) \mathrm{S}(1)$ integrated intensity map (red; Neumayer et al. 2007). The ALMA CO(3-2) and CO(6-5) maps cover a field of view of $24^{\prime \prime}$ and $12^{\prime \prime}$, and have a resolution of $\sim 0$." 3 (or 5 pc) resolution. The distribution of molecular hydrogen as traced by the $\mathrm{H}_{2}$ line is mostly contained within a field of view of $3^{\prime \prime}(54 \mathrm{pc})$. The cross sign in the center of the image shows the AGN position at R.A. $=13^{\mathrm{h}} 25^{\mathrm{m}} 27^{\mathrm{S}} .615$, decl. $=-43^{\circ} 01^{\prime} 08^{\prime \prime} .805$ (Ma et al. 1998).

bar which funnels gas to radii close to their SMBHs (Shlosman et al. 1989).

Centaurus $\mathrm{A}(\mathrm{Cen} \mathrm{A})$ is a radio source associated with the giant elliptical NGC 5128 at a distance of only $D \simeq 3.8 \mathrm{Mpc}$ (Harris et al. 2010, where $1^{\prime \prime}=18 \mathrm{pc}$ ). Although characterized by a bolometric luminosity $\left(\sim 2 \times 10^{43} \mathrm{erg} \mathrm{s}^{-1}\right.$; Israel 1998) and accretion rate $\left(\dot{M}_{\text {Bondi }}=6.4 \times 10^{-4} M_{\odot} \mathrm{yr}^{-1}\right.$ and a Bondi efficiency of $\sim 0.23 \%$, Evans et al. 2004) more modest than other powerful radio galaxies, it is by far the nearest and possibly best studied one (for reviews, see Israel 1998; Morganti 2010). Because of its proximity, it is the best target among the class of powerful radio galaxies for detailed studies of the feeding mechanisms of an AGN and feedback on the surrounding molecular gas. Cen A represents a particularly interesting case of an elliptical galaxy that was replenished recently (a few $10^{8}$ years) by gas from an external source (e.g., Struve et al. 2010).

Projected toward the inner several hundred parsec of Cen A, the following components have been identified (see Figure 1): (i) molecular gas at large radii $(>1 \mathrm{kpc})$ as traced by $\mathrm{CO}$ transitions (e.g., Phillips et al. 1987; Eckart et al. 1990; Rydbeck et al. 1993; Liszt 2001; Espada et al. 2009) and corresponding to kiloparsec-scale spiral features (Espada et al. 2012). This component is associated with the dust lane and is seen to be coextensive with other components of the interstellar medium, such as $\mathrm{H} \alpha$ (e.g., Nicholson et al. 1992), near-infrared (Quillen et al. 1993), submillimeter (e.g., Hawarden et al. 1993; Leeuw et al. 2002), and mid-IR continuum emission (e.g.,
Mirabel et al. 1999; Quillen et al. 2006). (ii) A circumnuclear gaseous disk (CND) or torus of $\sim 400 \mathrm{pc}$ extent $\left(\sim 24^{\prime \prime}\right)$ and a position angle P.A. $=155^{\circ}$, perpendicular to the inner jet, at least in projection (Espada et al. 2009) (see Figure 1(a)). The estimated total gas mass in this component is $8.4 \times 10^{7} M_{\odot}$ (Israel et al. 2014). The CO emission line is brightest at the edges of the disk at the NW and SE of the AGN, and Espada et al. (2009) reported a possible gap of $\mathrm{CO}(2-1)$ emission in the inner $r<80 \mathrm{pc}$ (see also Figure 1(a)). ALMA science verification $\mathrm{CO}(2-1)$ observations, also with tens of parsec resolution, showed similar results (Espada 2013). (iii) A nuclear disk ( $40 \mathrm{pc}$ diameter, or $\left.2^{\prime \prime}\right)$ containing ionized and molecular gas presumably feeding the nuclear massive object (e.g., Marconi et al. 2001, 2006). While ionized gas shows an elongated distribution along the jet and is likely related to it, the molecular hydrogen as traced by $\mathrm{H}_{2}(J=1-0) \mathrm{S}(1)$ seems to be mostly unperturbed within an irregular nuclear disk-like structure (see Figure 1(b)), and with an S-shaped velocity field (Neumayer et al. 2007). (iv) Absorption lines toward the continuum source detected in H I (e.g., Roberts 1970; van der Hulst et al. 1983; Sarma et al. 2002; Espada et al. 2010) and molecular lines (e.g., Israel et al. 1990; Wiklind \& Combes 1997; Eckart et al. 1999; Espada et al. 2010).

A fundamental question regarding this object, though, is how the gas in the nuclear disk at parsec scales is replenished by molecular gas in the CND. Previous $\mathrm{CO}(2-1)$ observations seemed to indicate that there was a lack of emission (Figure 1; Espada et al. 2009), which suggested a lack of molecular gas in 
Table 1

Main Parameters of the ALMA Observations

\begin{tabular}{|c|c|c|}
\hline & Band 7 & Band 9 \\
\hline Date & 2014 Jul 07 & 2014 Apr 14 \\
\hline Number of antennas & 33 & 34 \\
\hline Unprojected baselines (m) & $20-650$ & $20-558$ \\
\hline Time on source (min.) & 15 & 54 \\
\hline FWHM of primary beam $\left({ }^{\prime \prime}\right)$ & 16.9 & 8.4 \\
\hline FWHM of synthesized beam & $0 . " 36 \times 0 . " 29(6.5 \times 5.2 \mathrm{pc})$, P.A. $=70^{\circ}$ & $0 " .23 \times 0 . \prime 16(4.1 \times 2.9$ pc $)$, P.A. $=48^{\circ}$ \\
\hline Spectral window center frequency $(\mathrm{GHz})$ & $345.302,355.320,356.734$ & $691.473,693.300,705.209,708.877$ \\
\hline Lines/rest frequency $(\mathrm{GHz})$ & $\begin{array}{l}\mathrm{CO}(3-2) 345.796, \mathrm{HCN}(4-3) 354.505 \\
\mathrm{HCO}^{+}(4-3) 356.734\end{array}$ & $\mathrm{CO}(6-5) 691.473, \mathrm{HCN}(8-7) 708.877$ \\
\hline Bandwidth per baseband & $0.9375 \mathrm{GHz}\left(790 \mathrm{~km} \mathrm{~s}^{-1}\right)$ & $1.875 \mathrm{GHz}\left(790 \mathrm{~km} \mathrm{~s}^{-1}\right)$ \\
\hline Spectral resolution & $244.141 \mathrm{kHz}\left(0.4 \mathrm{~km} \mathrm{~s}^{-1}\right)$ & $488.281 \mathrm{kHz}\left(0.4 \mathrm{~km} \mathrm{~s}^{-1}\right)$ \\
\hline rms continuum & $2.2 \mathrm{mJy}$ beam $^{-1}$ & $16.6 \mathrm{mJy}^{\text {beam }^{-1}}$ \\
\hline \multirow[t]{2}{*}{$\mathrm{rms}$ in $10 \mathrm{~km} \mathrm{~s}^{-1}$} & $1.3 \mathrm{mJy}$ beam $^{-1}(\mathrm{CO}(3-2))$ & $5.8 \mathrm{mJy}$ beam $^{-1}(\mathrm{CO}(6-5))$ \\
\hline & $1.8 \mathrm{mJy}$ beam $^{-1}\left(\mathrm{HCN}(4-3), \mathrm{HCO}^{+}(4-3)\right)$ & \\
\hline Calibrators (ampl./bandpass/phase) & Titan/3C 279/J1427-4206 & Titan/3C 279/J1427-4206 \\
\hline
\end{tabular}

the center or a sudden change in the physical properties of the gas. Historically observing the higher transitions of $\mathrm{CO}$ or other dense gas tracers has been very challenging technically, and the problem is compounded for Cen A due to its location in the southern sky $\left(\right.$ decl. $\left.=-42^{\circ}\right)$. ALMA finally provides the missing data, and in this paper we present $\mathrm{CO}(3-2)$, $\mathrm{HCO}^{+}(4-3), \mathrm{HCN}(4-3)$, and $\mathrm{CO}(6-5)$ emission line maps with $5 \mathrm{pc}$ resolution toward the CND. These form an excellent suite of transition lines to probe the warm and dense molecular gas. The critical densities of $\mathrm{CO}(3-2), \mathrm{HCO}^{+}(4-3), \mathrm{CO}(6-5)$, and $\mathrm{HCN}(4-3)$ are $8.4 \times 10^{3} \mathrm{~cm}^{-3}, 1.0 \times 10^{6} \mathrm{~cm}^{-3}$, $1.8 \times 10^{6} \mathrm{~cm}^{-3}$, and $8.5 \times 10^{6} \mathrm{~cm}^{-3}$, respectively.

The paper is organized as follows. We introduce our ALMA observations and data reduction in Section 2. In Section 3, we focus on the identification of the different components, answer whether there is a gap in the molecular gas, and study their major physical properties. We interpret these results in order to shed light on the following questions. (i) What are the mechanisms that drive the gas from kiloparsec to parsec scales (in Section 4)? (ii) What is the geometry between the CND and the jet (in Section 5)? (iii) How do the molecular gas properties of the CND compare to those in numerical simulations of the multiphase ISM around SMBHs (in Section 6)? (iv) With our molecular line maps at hand, what are the chemical properties of the molecular gas close to the AGN (in Section 7)? Finally, we summarize our results in Section 8.

\section{Observations and Data Reduction}

We present single pointing observations toward the center of the molecular CND of Cen A, observed as part of project 2012.1.00225.S (PI: D. Espada). The chosen angular resolution was $\sim 0$.'3 (5 pc) for the band 7 and 9 observations. The angular resolution obtained in band 7 and 9 is almost a factor of a hundred better in area than the resolution obtained in previous SMA or ALMA CO(2-1) maps (Espada et al. 2009, 2012, 2013), and comparable to the warm $\mathrm{H}_{2}$ map resolution (2 pc) by Neumayer et al. (2007) using VLT/SINFONI.

Observations took place in 2014 July and April for the band 7 and 9 observations, with 33 and 34 antennas, respectively, and unprojected baseline lengths spanning $20-650 \mathrm{~m}$ and $20-558 \mathrm{~m}$. The maximum angular scale (defined as $0.6 \lambda / L_{\min }$, where $\lambda$ is the wavelength and $L_{\min }$ the minimum baseline) for which we recover most of the flux is $5^{\prime \prime}(90 \mathrm{pc})$ for band 7 and $3^{\prime \prime}(54 \mathrm{pc})$ for band 9.

The $\mathrm{CO}(3-2) \quad\left(\nu_{\text {rest }}=345.796 \mathrm{GHz}\right)$ emission line was observed simultaneously with $\mathrm{HCO}^{+}(4-3)\left(\nu_{\text {rest }}=356.734 \mathrm{GHz}\right)$ in the lower sideband and $\mathrm{HCN}(4-3) \quad\left(\nu_{\text {rest }}=354.505 \mathrm{GHz}\right)$ in the upper sideband. $\mathrm{CO}(6-5)\left(\nu_{\text {rest }}=691.473 \mathrm{GHz}\right)$ was also observed in the lower sideband simultaneously with $\mathrm{HCN}(8-7)$ $\left(\nu_{\text {rest }}=708.877 \mathrm{GHz}\right)$, but the latter was not detected in emission. One execution of $2 \mathrm{hr}$ was carried out in band 7 , and three executions of $2 \mathrm{hr}$ each for band 9 in different Local Sidereal Time ranges. The setups of our interferometric observations are summarized in Table 1 . The systemic velocity of Cen $\mathrm{A}$ is $V_{\text {sys }}=$ $541.6 \mathrm{~km} \mathrm{~s}^{-1}$ (Espada et al. 2010).

The field of view is characterized by a half-power beam width (HPBW) for the primary beam of an ALMA $12 \mathrm{~m}$ antenna of 16".9 (304 pc) in band 7 and 8!" 4 (151 pc) in band 9 . The bandwidth chosen was $937.5 \mathrm{MHz}$ for band 7 and $1.875 \mathrm{GHz}$ for band 9 (both corresponding to $\sim 800 \mathrm{~km} \mathrm{~s}^{-1}$ ), and the channel width of 244 and $488 \mathrm{kHz}$ for spectral windows at bands 7 and 9 (or $\sim 0.4 \mathrm{~km} \mathrm{~s}^{-1}$ ), respectively. Enough line-free channels are present to subtract the continuum emission.

The CASA package (McMullin et al. 2007) was used for data reduction. The CASA version used was 4.2.1 (r29048). Each execution was calibrated separately by the East Asian ALMA Regional Center (EA-ARC) and followed the standard ALMA calibration scheme. A priori calibration tables were created for Water Vapor Radiometer phase correction and atmospheric calibration. Then bandpass, gain (amplitude, phase), and flux calibrations were applied. Titan and 3C 279 were chosen for absolute flux and bandpass calibration, respectively. An initial gain calibration was performed using $\mathrm{J} 1321-4342$ or $\mathrm{J} 1427-4206$, at angular distances of $1^{\circ}$ and $11^{\circ}$ from the target. Self-calibration was then done by us in phase and amplitude using the bright and compact continuum source of Cen A, and only using line-free channels.

The fluxes obtained in band 7 at $345.6 \mathrm{GHz}$ on 2014 July 7 were $6.46 \mathrm{Jy}$ for $3 \mathrm{C} 279$ and $2.42 \mathrm{Jy}$ for J1427-4206. This is in very good agreement with fluxes in the ALMA source catalog database at similar dates: at $343.48 \mathrm{GHz}$, the fluxes were $6.60 \mathrm{Jy}$ for 3C 279 and $2.38 \mathrm{Jy}$ for $1427-4206$, on 2014 July 6 and June 30, respectively. The fluxes obtained in band 9 at 691.1 GHz on 2014 April 14 were 4.5 Jy for 3C 279 and 2.3 Jy 
for J1427-4206, and all three executions show similar results. These fluxes are also consistent with the measurements in the ALMA source catalog in that band $(691.1 \mathrm{GHz})$ on 2014 April 25, 4.35-4.99 Jy for 3C 279, and $1.96 \mathrm{Jy}$ for J1427-4206. Note that J1321-4342 was not monitored as frequently by the ALMA project, and it is therefore not useful for a comparison. We thus estimate absolute flux uncertainties of the order of $5 \%$ for band 7 and $10 \%-20 \%$ for band 9 .

Once calibration for each data set was completed, we concatenated all executions and proceeded to imaging also using CASA with task CLEAN. First, we imaged the continuum emission using line-free channels. We cleaned the continuum image using the multifrequency synthesis technique (MFS), and using the Briggs weighting scheme with a robust parameter 0.5 . The obtained noise levels are $2.2 \mathrm{mJy} /$ beam and $16.6 \mathrm{mJy} /$ beam for the 350 and $698 \mathrm{GHz}$ continuum maps, respectively. To image the emission lines, and prior to the cleaning, we used task UVCONTSUB to subtract the continuum using as baseline the line-free channels and an order of 1 in the fitting polynomial function. Since the continuum emission is so strong in this source, we paid special attention that continuum subtraction was done correctly by checking for possible artifacts in the line-free channels. The weighting scheme for imaging of the lines we used was also Briggs and robust parameter 0.5. In the channel maps, we binned the data to $10 \mathrm{~km} \mathrm{~s}^{-1}$, which is enough to resolve spectrally the $\sim 400 \mathrm{~km} \mathrm{~s}^{-1}$ velocity width of the circumnuclear gas. Note that velocities are expressed throughout this paper with respect to the kinematic local standard of rest (LSRK) using the radio convention.

The angular resolution expressed as the FWHM is 0 " $36 \times 0$ ". $29(6.5 \times 5.2 \mathrm{pc})$ with a major axis P.A. $=70^{\circ}$ in our band 7 observations, and 0. . $23 \times 0$." $16(4.1 \times 2.9 \mathrm{pc})$ with a major axis P.A. $=48^{\circ}$ in our band 9 observations. The mean rms noise levels are $1.3 \mathrm{mJy}_{\text {beam }}^{-1}$ and $5.8 \mathrm{mJy} \mathrm{beam}^{-1}$ for the Briggs weighted band 7 and band 9 channel maps with a channel width of $10 \mathrm{~km} \mathrm{~s}^{-1}$. The channel maps presentation was conducted in MIRIAD (Sault et al. 1995). The task IMMOMENTS in CASA was used to calculate the integrated intensity maps, intensity-weighted velocity field, and velocity dispersion distributions (the latter two clipped at five times the rms noise).

\section{Results}

We present in this section the results obtained from the 350 and $698 \mathrm{GHz}$ continuum maps, as well as the $\mathrm{CO}(3-2)$, $\mathrm{HCO}^{+}(4-3), \mathrm{HCN}(4-3)$, and $\mathrm{CO}(6-5)$ emission spectral line maps.

\subsection{0 and $698 \mathrm{GHz}$ Continuum Emission}

The continuum emission was found to be unresolved at $350 \mathrm{GHz}$ and $698 \mathrm{GHz}$. The peak coordinates for the $350 \mathrm{GHz}$ and $698 \mathrm{GHz}$ continuum maps were calculated using a twodimensional fit and are very close to each other. To our angular resolution, the $350 \mathrm{GHz}$ and $698 \mathrm{GHz}$ peaks are identical and located at R.A. $=13^{\mathrm{h}} 25^{\mathrm{m}} 27^{\mathrm{s}} .615$, decl. $=-43^{\circ} 01^{\prime} 08^{\prime \prime} 805$. The uncertainty is at most one-tenth of the synthesized beam. This is in perfect agreement with the position of the AGN found by Ma et al. (1998) using Very Long Baseline Interferometry (VLBI). We will keep this position as our reference for the AGN location. On 2014 July 7, the flux for the $350 \mathrm{GHz}$
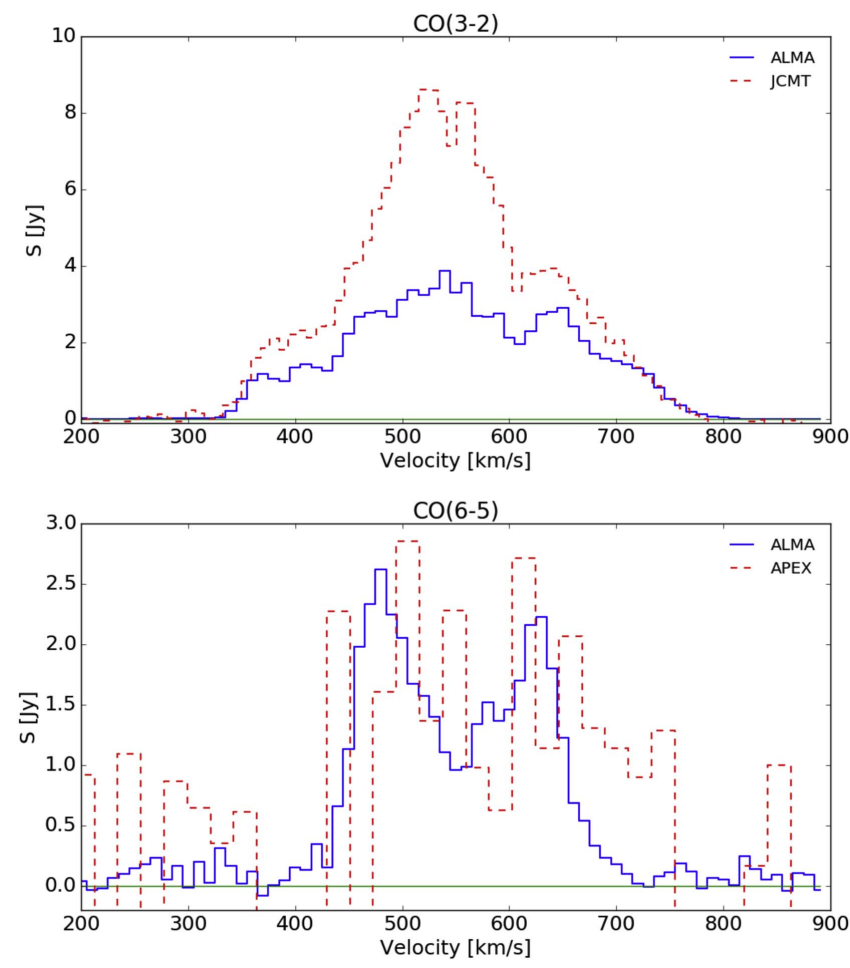

Figure 2. $\mathrm{CO}(3-2)$ and $\mathrm{CO}(6-5)$ spectra. The $y$-axis is in $\mathrm{Jy}$, from -0.1 to $10 \mathrm{Jy}$ and -0.2 to $3.0 \mathrm{Jy}$. The $x$-axis shows radio LSR velocity in units of $\mathrm{km} \mathrm{s}^{-1}$ from 200 to $900 \mathrm{~km} \mathrm{~s}^{-1}$. Note that these two spectra include Cen A emission from detected regions in the data cubes (see Figures 5 and 10 for $\mathrm{CO}(3-2)$ and $\mathrm{CO}(6-5)$, respectively) and exclude the spatially unresolved region exhibiting absorption at the center of the galaxy. The dashed lines show JCMT $\mathrm{CO}(3-2)$ and APEX $\mathrm{CO}(6-5)$ spectra from Israel et al. (2014) for comparison.

continuum was measured to be $8.0 \pm 0.1 \mathrm{Jy}$. The $698 \mathrm{GHz}$ continuum flux was found to be $7.7 \pm 0.1 \mathrm{Jy}$ on 2014 April 14 .

\section{2. $\mathrm{CO}(3-2)$ Emission}

In Figure 2, we present the $\mathrm{CO}(3-2)$ spectrum integrated over detected regions in the inner $24^{\prime \prime}$ and excluding the center of the image where absorption lines are found toward the (unresolved) continuum emission in the velocity range from 540 to $620 \mathrm{~km} \mathrm{~s}^{-1}$. The profile is relatively flat and shows two or probably three peaks, at about 480,550 , and $650 \mathrm{~km} \mathrm{~s}^{-1}$. High red- and blueshifted velocity components are also seen at $V<420 \mathrm{~km} \mathrm{~s}^{-1}$ and $V>680 \mathrm{~km} \mathrm{~s}^{-1}$, which were not as clearly seen in previously obtained $\mathrm{CO}$ interferometric data due to a lack of angular resolution and sensitivity. In order to illustrate where the missing flux is important, we also plot in Figure 2 the $15 \mathrm{~m}$ James Clerk Maxwell Telescope (JCMT) $\mathrm{CO}(3-2)$ profile (Israel et al. 2014) for comparison. Note that the ALMA $\mathrm{CO}(3-2)$ spectrum presented in Figure 2 was obtained after correcting the $\mathrm{CO}(3-2)$ data by the different beam response of the JCMT (14" HPBW) in order to be able to compare.

The integrated flux measurement of the ALMA CO(3-2) line is $1102 \pm 6 \mathrm{Jy} \mathrm{km} \mathrm{s}^{-1}$, with no primary beam correction, and $1552 \pm 8 \mathrm{Jy} \mathrm{km} \mathrm{s}^{-1}$ with primary beam correction. Error estimates correspond to the nominal value, but note that uncertainties are larger due to absolute flux uncertainties and filtered flux. Correcting by the different beam response of the JCMT, we obtain $880 \mathrm{Jy} \mathrm{km} \mathrm{s}^{-1}$. This is to be compared with the flux of $1600 \mathrm{Jy} \mathrm{km} \mathrm{s}^{-1}$ obtained directly from the JCMT 


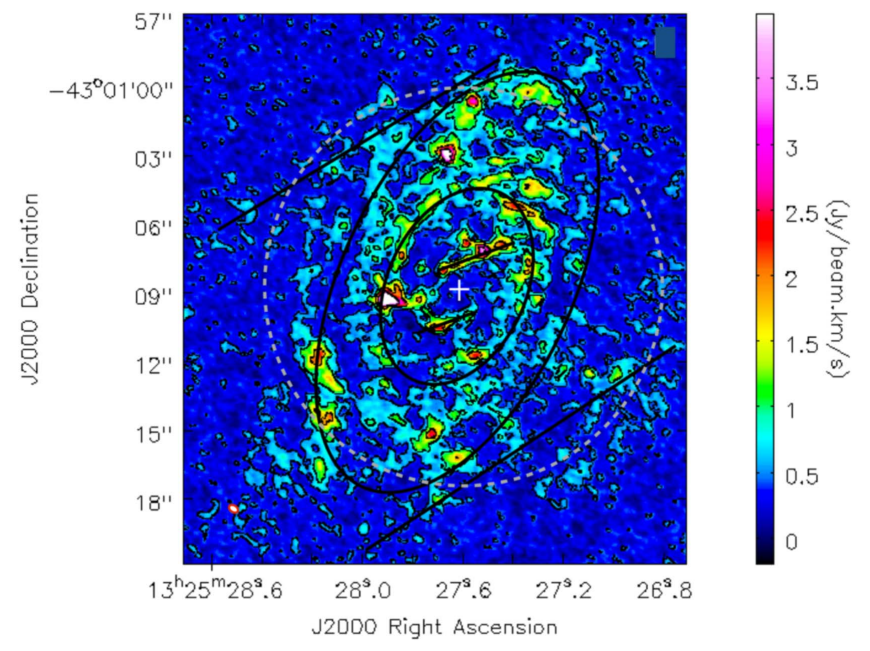

Figure 3. $\mathrm{CO}(3-2)$ integrated intensity map of the CND of Cen A. Contour levels are at $0.44,0.9,1.8$, and $3 \mathrm{Jy}_{\text {beam }}^{-1} \mathrm{~km} \mathrm{~s}^{-1}$. The synthesized beam $\left(0.36 \times 0\right.$. " 29 , P.A. $\left.=70^{\circ} .44\right)$ is indicated by an ellipse in the lower-left corner of the plot, and the color scale is shown beside the plot, also in units of Jy beam ${ }^{-1} \mathrm{~km} \mathrm{~s}^{-1}$. The cross in the center of the image shows the position of the AGN, the (gray) dotted line the primary beam at $345 \mathrm{GHz}$ $(\mathrm{HPBW}=16 . .9)$, and the two (black) ellipses the CND and the nuclear ring, with semimajor axes of $10^{\prime \prime} \times 5^{\prime \prime}$ and $4 !^{\prime \prime} 5 \times 3^{\prime \prime}$, respectively, and both with a P.A. $=155^{\circ}$. The two lines outside the CND correspond to gas at larger radii seen in projection associated with the spiral arms and parallelogram features aligned along P.A. $=120^{\circ}$ and at velocities close to the systemic velocity. The two parallel lines inside the inner ring correspond to two nuclear filaments (loci of shocks) with lengths of $4^{\prime \prime}$ (NW of the AGN) and $3^{\prime \prime}$ (SE of the AGN), with P.A. $=110^{\circ}$ and with an offset between them of $2^{\prime \prime}$.

$\mathrm{CO}(3-2)$ spectrum in Figure 2. The absorption line flux was estimated to be $220 \mathrm{Jy} \mathrm{km} \mathrm{s}^{-1}$ (Israel et al. 2014). Therefore, our map recovers $\sim 50 \%$ of the flux. From Figure 2, we can see that we are missing emission preferentially around the systemic velocity. The extended emission that arises from the disk on large spatial scales is likely one of the most important contributors to the missing flux due to the lack of short spacings.

Figures 1(b) and 3 show the $\mathrm{CO}(3-2)$ integrated intensity map. The extent of the CND is represented by an ellipse with major and minor axes of $20^{\prime \prime} \times 10^{\prime \prime}$, or $360 \mathrm{pc} \times 180 \mathrm{pc}$ in linear scale (without taking into account any projection effect), and a position angle of P.A. $=155^{\circ}$. Note that there is emission beyond the primary beam of the central pointing at $345 \mathrm{GHz}$. Figure 4 shows a simplified scheme that illustrates the main molecular components within the CND that are discussed in this paper (see Section 3.6).

In Figure 5, we show the $\mathrm{CO}(3-2)$ channel maps over the CND of Cen A. The map covers the inner $24^{\prime \prime}(432 \mathrm{pc})$ and a velocity interval from 315 to $790 \mathrm{~km} \mathrm{~s}^{-1}$ in $20 \mathrm{~km} \mathrm{~s}^{-1}$ bins. Note that no primary beam correction was performed in these maps. Figure 6 shows the enclosed $12^{\prime \prime}$ area to highlight the different inner components within the CND. The $\mathrm{CO}(3-2)$ channel maps show blueshifted $\left(\sim 315-495 \mathrm{~km} \mathrm{~s}^{-1}\right)$ emission on the SE side and redshifted $\left(\sim 615-790 \mathrm{~km} \mathrm{~s}^{-1}\right)$ emission on the NW side. The full width at zero intensity (FWZI) is $\Delta V \simeq 475 \pm 7 \mathrm{~km} \mathrm{~s}^{-1}$.

The higher velocity components in the spectrum (see Figure 2) at $V<420 \mathrm{~km} \mathrm{~s}^{-1}$ and $V>680 \mathrm{~km} \mathrm{~s}^{-1}$ are located at the edges of the CND. The molecular gas at large radii (likely $\geqslant 1 \mathrm{kpc}$, although seen in projection in the field of view) associated with the kiloparsec-scale spiral features (see Section 1, emission around the parallel lines in Figures 3 and 5) can be discerned

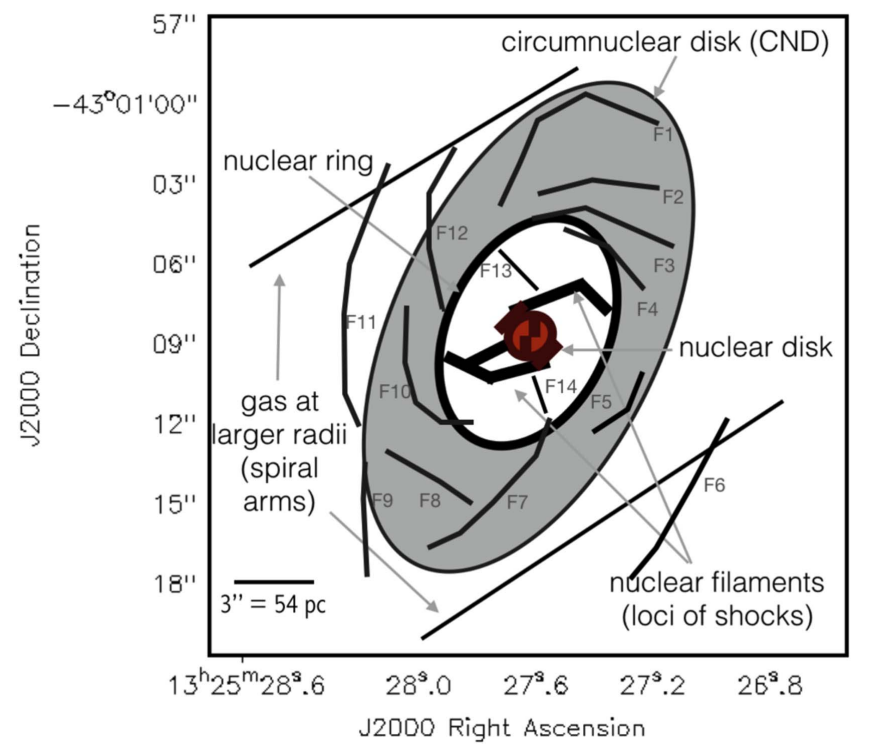

Figure 4. Scheme showing the main molecular components that are present in the CND of Cen A. The field of view is as in Figure 3. Regions marked in black/gray indicate those traced by the transitions presented in this paper, and in red those traced by the $1-0 \mathrm{~S}(1) \mathrm{H}_{2}$ line in Neumayer et al. (2007). The main filamentary structures within the CND as seen in the channel maps are also indicated as black lines. An identifier for each filament with format $\mathrm{FXX}$ is provided.

as very extended components in the velocity range from $495 \mathrm{~km} \mathrm{~s}^{-1}$ to $635 \mathrm{~km} \mathrm{~s}^{-1}$ : from $495 \mathrm{~km} \mathrm{~s}^{-1}$ to $535 \mathrm{~km} \mathrm{~s}^{-1}$ toward the NE with respect to the center, and from $515 \mathrm{~km} \mathrm{~s}^{-1}$ to $635 \mathrm{~km} \mathrm{~s}^{-1}$ toward the SW. Note that these extended components are likely the most affected by missing flux in this interferometric experiment.

There are multiple filaments within the previously discovered CND that are apparent in the maps we present in this paper. The longest feature, extending more than $15^{\prime \prime}$ (or $270 \mathrm{pc}$ ), is located just at the eastern edge of the CND (see, for example, the channel at $V=495 \mathrm{~km} \mathrm{~s}^{-1}$ in Figure 5). Note that some filamentary structures may in part be caused by projection effects, especially in the channels from 495 to $595 \mathrm{~km} \mathrm{~s}^{-1}$ (close to the systemic velocity), where emission from the CND and from gas expected to arise from larger galactocentric radii $(r>1 \mathrm{kpc})$ might be projected along a similar line of sight. Multiple streamers on scales of tens to one hundred parsec scale also exist within the CND, which form a ring-like structure (nuclear ring) with a diameter of $9^{\prime \prime} \times 6^{\prime \prime}$ $(160 \mathrm{pc} \times 108 \mathrm{pc})$ and a P.A. $=155^{\circ}$. The nuclear ring can be discerned in the map showing the integrated emission (Figure 3), and in channels from $415 \mathrm{~km} \mathrm{~s}^{-1}$ to $675 \mathrm{~km} \mathrm{~s}^{-1}$ (Figure 5). Inside the ring, there are two filaments elongated along a position angle P.A. $=120^{\circ}$ and separated by $2^{\prime \prime}$ to the SE and NW from the AGN location. More details on the different components are provided in Section 3.6.

Figure 7 presents the intensity-weighted velocity field (left panel) and the velocity dispersion maps (right). The receding side of the CND is to the NW and the approaching side to the SE. As discussed in Espada et al. (2009), there are multiple components along the line of sight and the velocity field should be taken with caution when it overlaps with projected emission arising from gas at larger radii. This is most important at the edges of the CND to the NW and SE. There are deviations with respect to a simple circularly rotating coplanar disk because the velocity field shows an S-shape distribution (see Section 4). 


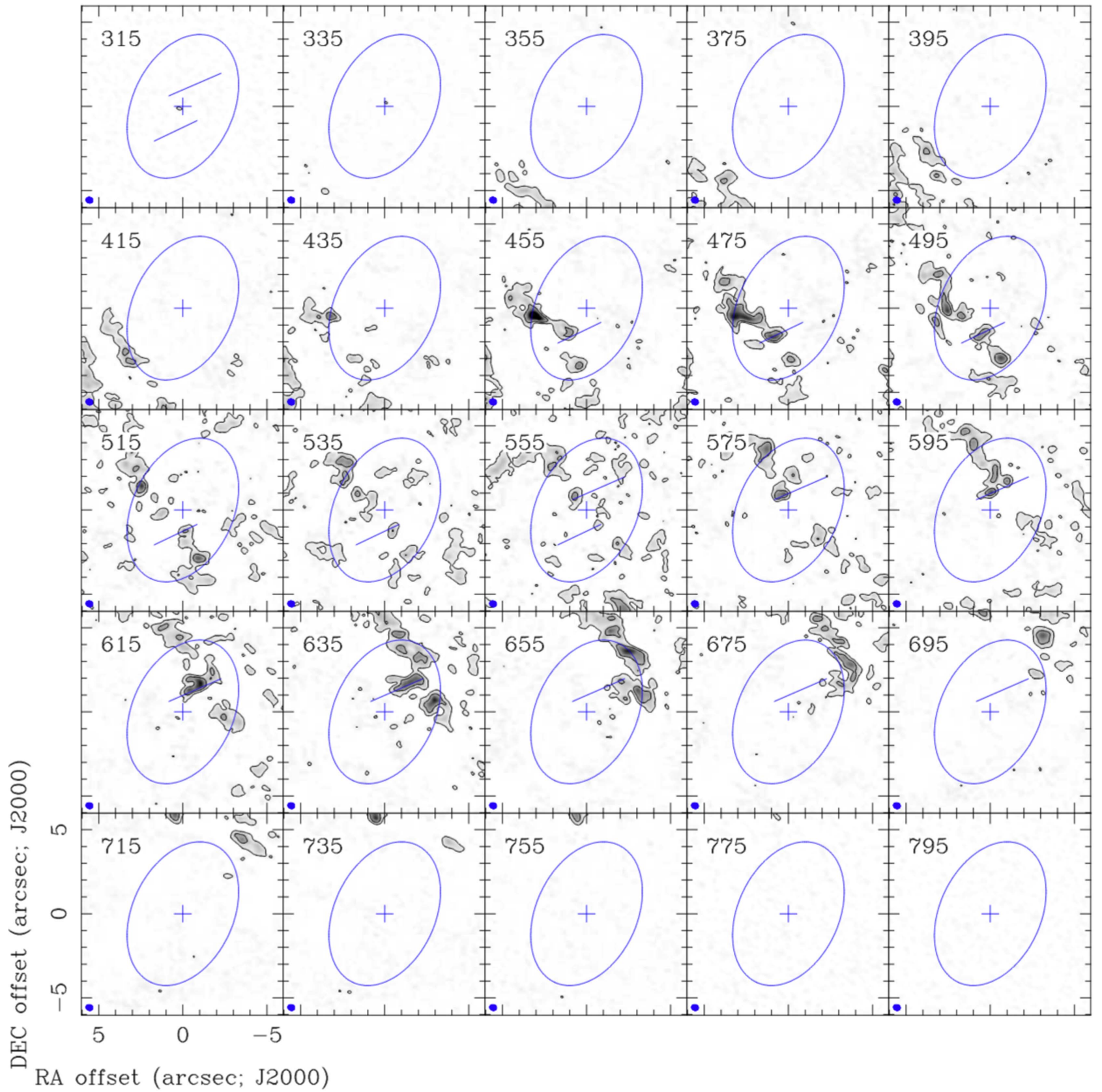

Figure 5. CO(3-2) channel maps of the CND of Cen A in the LSR velocity range $V=315-795 \mathrm{~km} \mathrm{~s}^{-1}$ in $20 \mathrm{~km} \mathrm{~s}^{-1}$ bins. The size of the maps is $24^{\prime \prime}$. The velocities are shown in the upper-left corner and the synthesized beam is shown at the bottom-left corner of each panel. The rms noise of an individual channel is $0.92 \mathrm{mJy}_{\text {beam }}{ }^{-1}$. The contour levels are $5 \sigma, 25 \sigma$, and $100 \sigma$. The cross sign shows the galaxy's AGN position at R.A. $=13^{\mathrm{h}} 25^{\mathrm{m}} 27^{\mathrm{s}} 615$, decl. $^{2}=-43^{\circ} 01^{\prime} 08^{\prime \prime} .805$ (Ma et al. 1998). See Figure 4 for a description of the symbols representing the main molecular components of the CND of Cen A.

The velocity dispersion map shows that there is a large range of values within the CND, from a few $\mathrm{km} \mathrm{s}^{-1}$ to tens of $\mathrm{km} \mathrm{s}^{-1}$. Note that in this plot we excluded the six channels at velocities $<60 \mathrm{~km} \mathrm{~s}^{-1}$ from the systemic velocity (i.e., $545 \mathrm{~km} \mathrm{~s}^{-1}$ ) to avoid contamination by the molecular gas component at $r \geqslant 1 \mathrm{kpc}$ seen in projection. The velocity width of this component is much narrower $\left(\sim 140 \mathrm{~km} \mathrm{~s}^{-1}\right)$ than the CND in the field of view, so we are effectively removing most of its contribution. In general, the molecular gas has low velocity dispersions of $\sim 5-10 \mathrm{~km} \mathrm{~s}^{-1}$, but especially in the outermost parts of the CND to the NW and SE, we see that the velocity dispersion increases, and there are velocity dispersions of up to $30-40 \mathrm{~km} \mathrm{~s}^{-1}$. Other regions such as the nuclear filaments have larger velocity dispersions as well. The large velocity dispersions there become apparent in the position-velocity $(\mathrm{P}-\mathrm{V})$ diagrams, which are described next.

$\mathrm{P}-\mathrm{V}$ diagrams along several cuts are shown in Figure 8. Figures 8(a), (b), and (c) show the P-V diagrams along P.A. $=60^{\circ}, 120^{\circ}$, and $150^{\circ}$, respectively, all of them with widths of $6^{\prime \prime}$. These $\mathrm{P}-\mathrm{V}$ diagrams cross and are centered at the AGN position. From Figure 8(c) we estimate that the CND velocity gradient is typically $\Delta V / \Delta r=1.8 \mathrm{~km} \mathrm{~s}^{-1} \mathrm{pc}^{-1}$ (or $32 \mathrm{~km} \mathrm{~s}^{-1} \operatorname{arcsec}^{-1}$ ). At the edges of the CND, at about $-8^{\prime \prime}$ and $8^{\prime \prime}$ from the center, we can see in the $P-V$ diagrams along P.A. $=120^{\circ}$ and $150^{\circ}$ that there is a large radial velocity drop of $\sim 100 \mathrm{~km} \mathrm{~s}^{-1}$ toward more systemic values (see, for example, the region enclosed by the red boxes in the $150^{\circ} P-V$ diagram) both in the NW and SE. This is to be 


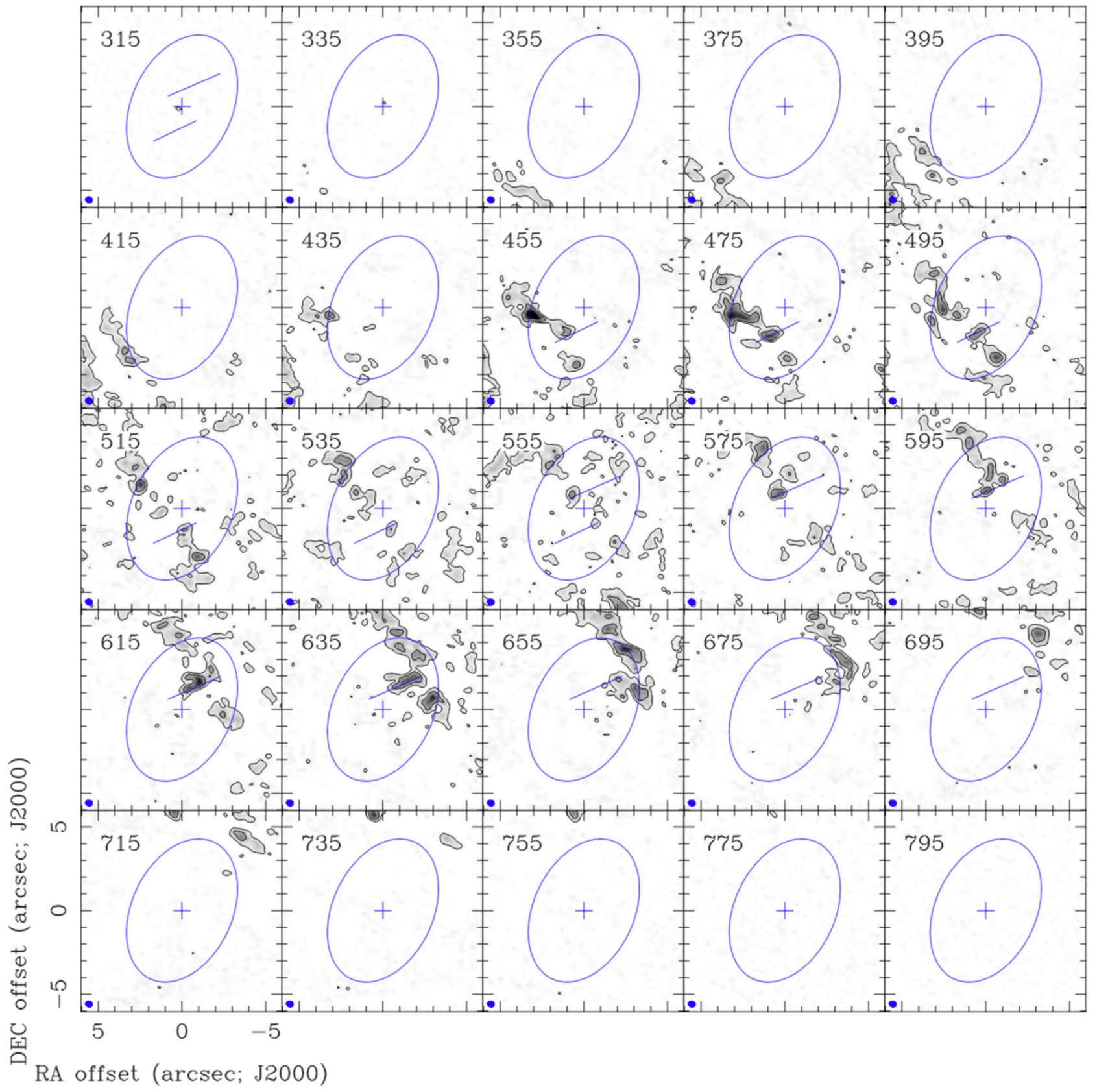

Figure 6. As Figure 5, but the size of the maps is $12^{\prime \prime}$ to highlight the inner molecular components.

compared with Espada et al. (2009), where the velocity gradient in the CND (up to $r \simeq 200 \mathrm{pc}$ ) was given as $1.2 \mathrm{~km} \mathrm{~s}^{-1} \mathrm{pc}^{-1}$, and $0.2 \mathrm{~km} \mathrm{~s}^{-1} \mathrm{pc}^{-1}$ in the outer parts of the disk located at $r>400 \mathrm{pc}$ and out to a few kiloparsecs. This velocity gradient for the CND agrees well with that quoted in this paper, although it is slightly lower mainly due to beam smearing in the previous SMA observations.

Apparent in all $\mathrm{P}-\mathrm{V}$ diagrams is that the nuclear filaments (within the inner $r=36 \mathrm{pc}$, or $2^{\prime \prime}$ ) inside the CND have an even steeper velocity gradient, which can be characterized by $\Delta V / \Delta r=3.4 \mathrm{~km} \mathrm{~s}^{-1} \mathrm{pc}^{-1}$ (or $62 \mathrm{~km} \mathrm{~s}^{-1} \operatorname{arcsec}^{-1}$ ). See, for example, the enclosed regions by the (red) boxes in Figure 8(a) and the (blue) box in Figure 8(c) for reference.

Figure 9 (left panel) shows the P-V diagrams obtained from $\mathrm{CO}(3-2)$ data compared with the compilation performed by
Espada et al. (2009), including $\mathrm{CO}(2-1)$ data, $\mathrm{H} \alpha$ data (Nicholson et al. 1992), and single-dish $\mathrm{CO}(3-2)$ data (Liszt 2001). In the inner region ( $r<0$ '2 ; see the right panel), one can distinguish the peculiar velocities of the two nuclear filaments, and in the outer regions $(r \sim 0$ '.14) the sudden drop in radial velocity of $\sim 100 \mathrm{~km} \mathrm{~s}^{-1}$. Data points for both receding and approaching sides are shown in these plots and they show good agreement. Note that a rotation curve obtained from $\mathrm{P}-\mathrm{V}$ diagrams has large uncertainties when non-circular motions are present (Sakamoto et al. 1999). Instead, an average across different directions is closer to the right rotation curve. Therefore, we confirm that the estimated rotation curve presented in Espada et al. (2009) is reasonably acceptable.

The decomposition of the $\mathrm{CO}(3-2)$ emission for the different components mentioned in this section is further explained in 

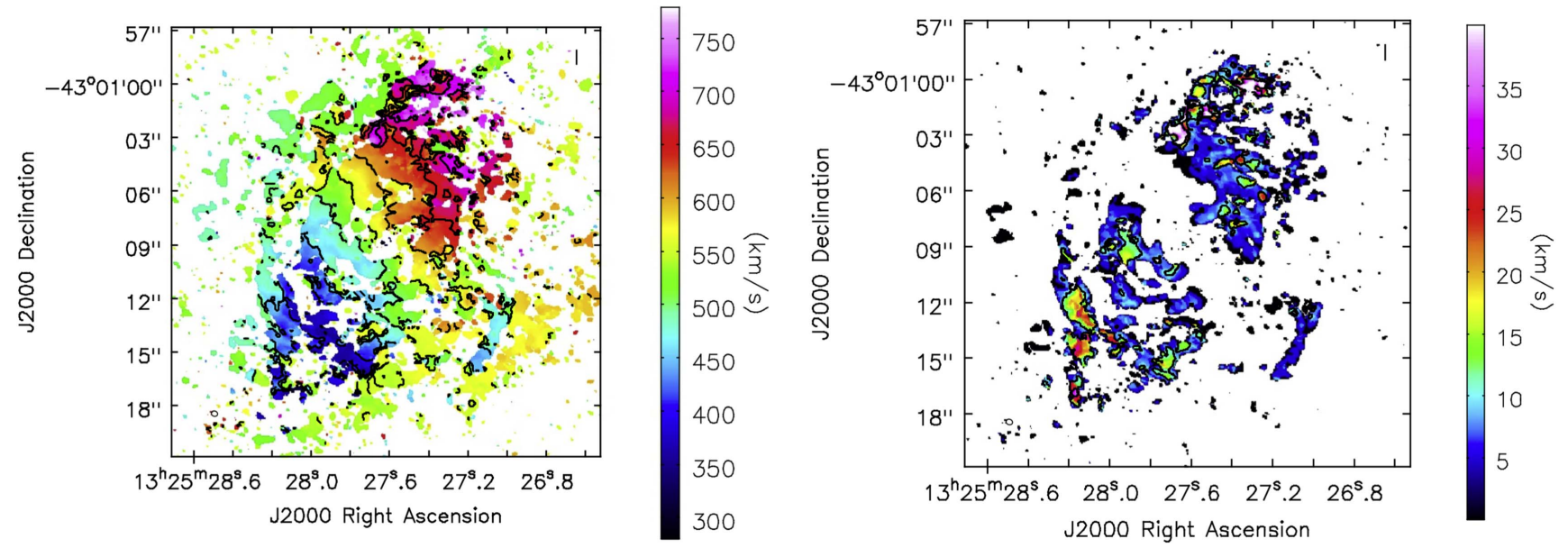

Figure 7. $\mathrm{CO}(3-2)$ (intensity weighted) velocity field and velocity dispersion maps of the CND of Cen A. In the velocity field map, contours are placed every $50 \mathrm{~km} \mathrm{~s}^{-1}$, from 350 to $700 \mathrm{~km} \mathrm{~s}^{-1}$. The color scale ranges from $280 \mathrm{~km} \mathrm{~s}^{-1}$ up to $780 \mathrm{~km} \mathrm{~s}^{-1}$. In the velocity dispersion map, we place contours at 10 and $30 \mathrm{~km} \mathrm{~s}$, and the color scale ranges from 0 to $40 \mathrm{~km} \mathrm{~s}^{-1}$. We excluded channels $<50 \mathrm{~km} \mathrm{~s}^{-1}$ from the systemic velocity $\left(V_{\mathrm{sys}}=541.6 \mathrm{~km} \mathrm{~s}^{-1}\right)$ to avoid contamination by the external molecular gas component. The synthesized beam $(0$ " $36 \times 0$ !"29) is shown at the bottom-left corner of each panel.

Section 3.6 and the main parameters are summarized in Table 2 .

\section{3. $\mathrm{CO}(6-5)$ Emission}

Figure 2 shows the ALMA $\mathrm{CO}(6-5)$ spectrum integrated over the detected regions, where we have excluded (as for the $\mathrm{CO}(3-2)$ spectrum) the absorption line toward the central continuum emission. We also show the APEX (Atacama Pathfinder EXperiment) $\mathrm{CO}(6-5)$ profile from Israel et al. (2014) for comparison. Note that within this field of view we mostly probe the molecular gas located in the ring and nuclear filaments. We can distinguish more clearly than in the $\mathrm{CO}(3-2)$ spectrum a double-peak structure. The high-velocity components in the $\mathrm{CO}(3-2)$ spectrum found at $V<420 \mathrm{~km} \mathrm{~s}^{-1}$ and $V>680 \mathrm{~km} \mathrm{~s}^{-1}$ are not present in the $\mathrm{CO}(6-5)$ spectrum partly because these are located in the external components of the $\mathrm{CND}$, which are attenuated because they are located outside the primary beam HPBW.

In order to estimate how affected the $\mathrm{CO}(6-5)$ map is by the missing zero spacing problem, we compare the fluxes with that of the single-dish measurement. The total flux measurement of the ALMA $\mathrm{CO}(6-5)$ data is $521 \pm 4 \mathrm{Jy} \mathrm{km} \mathrm{s}^{-1}$, with no primary beam correction. Again, the error estimates correspond to the nominal value without taking into account absolute flux uncertainties and filtered flux. We compare this directly with the flux obtained from observations from APEX since that antenna has a response similar to the individual ALMA $12 \mathrm{~m}$ antennas. The flux was found to be $787 \pm 187 \mathrm{Jy} \mathrm{km} \mathrm{s}^{-1}$ (Israel et al. 2014). This indicates that $\sim 30 \%$ of the flux is missing within this field of view, and reflects the compact nature of the $\mathrm{CO}(6-5)$ emission. Note that although the APEX spectrum is good enough to provide an integrated flux, it is probably too noisy for a channel to channel comparison. The $30 \%$ is an upper limit because the flux peaks are essentially equivalent within the uncertainties, and the flux difference arises mostly from the velocity range $650-750 \mathrm{~km} \mathrm{~s}^{-1}$, which may indicate that the APEX pointing was slightly offset toward the NW.

In Figure 10, we show the channel maps of the $\operatorname{CO}(6-5)$ emission line covering the inner $12^{\prime \prime}$ (same size as the $\mathrm{CO}(3-2)$ channel maps in Figure 6) and the velocity interval from 315 to
$795 \mathrm{~km} \mathrm{~s}^{-1}$ in $20 \mathrm{~km} \mathrm{~s}^{-1}$ bins. The blueshifted emission starts in channel $425 \mathrm{~km} \mathrm{~s}^{-1}$ at the SE of the AGN and the redshifted side ends at $705 \mathrm{~km} \mathrm{~s}^{-1}$ to the NW. The morphology is very similar to that of the $\mathrm{CO}(3-2)$ maps in this field of view. The two nuclear filamentary structures are clearly visible, as well as other filaments connected to them to the $\mathrm{N}$ and $\mathrm{S}$, and also, although weak (partly because they are close to the edge of the primary beam), some regions along the ring are detected.

Figures 11 and 12 show the $\mathrm{CO}(6-5)$ integrated intensity, velocity field, and velocity dispersion maps. Note that the resolution is slightly better (factor of three in area) in the $\mathrm{CO}(6-5)$ maps than in the $\mathrm{CO}(3-2)$ maps. Also, the confusion caused by the multiple components seen in $\mathrm{CO}(3-2)$ is not present in the $\mathrm{CO}(6-5)$ maps, and we can discern more clearly the structure and kinematics of the CND within its inner $8^{\prime \prime}$, and in particular the distribution of the nuclear filaments. Large velocity dispersions of $\sim 10-20 \mathrm{~km} \mathrm{~s}^{-1}$ can be found in some parts of the nuclear filaments.

The filaments on both sides of the AGN are composed by several Giant Molecular Clouds (GMCs) that are resolved with our synthesized beam. The closest projected distance of these clouds to the AGN is $1^{\prime \prime}$, or $\sim 20 \mathrm{pc}$. They form two nearly straight filaments, which are also kinematically distinct, i.e., deviations of typically $50 \mathrm{~km} \mathrm{~s}^{-1}$ can be seen from the rotational velocity of the $\mathrm{CND}$ at a given radius. However, we confirm that both filaments are seen to be slightly asymmetric with respect to each other. First, the GMCs are brighter by a factor of two in the NW filament than in the SE filament. Also, as in the $\mathrm{CO}(3-2)$ maps, while the NW filament is perfectly aligned, the one in the SE is slightly curved in the lower contours at the distant SE end. These asymmetries between the two sides indicate that the molecular gas is not well settled. $\mathrm{CO}(6-5)$ emission also exists to the $\mathrm{E}$ and $\mathrm{W}$ of the AGN and slightly farther from the AGN than the filaments, which connect the nuclear ring with the nuclear filaments. The eastern connecting point contains substantially more molecular gas than its western counterpart. The highest velocity dispersion regions are in the connecting points to the $\mathrm{E}$ and $\mathrm{W}$ of the AGN, and at the end of the filamentary structures as they approach the AGN. 

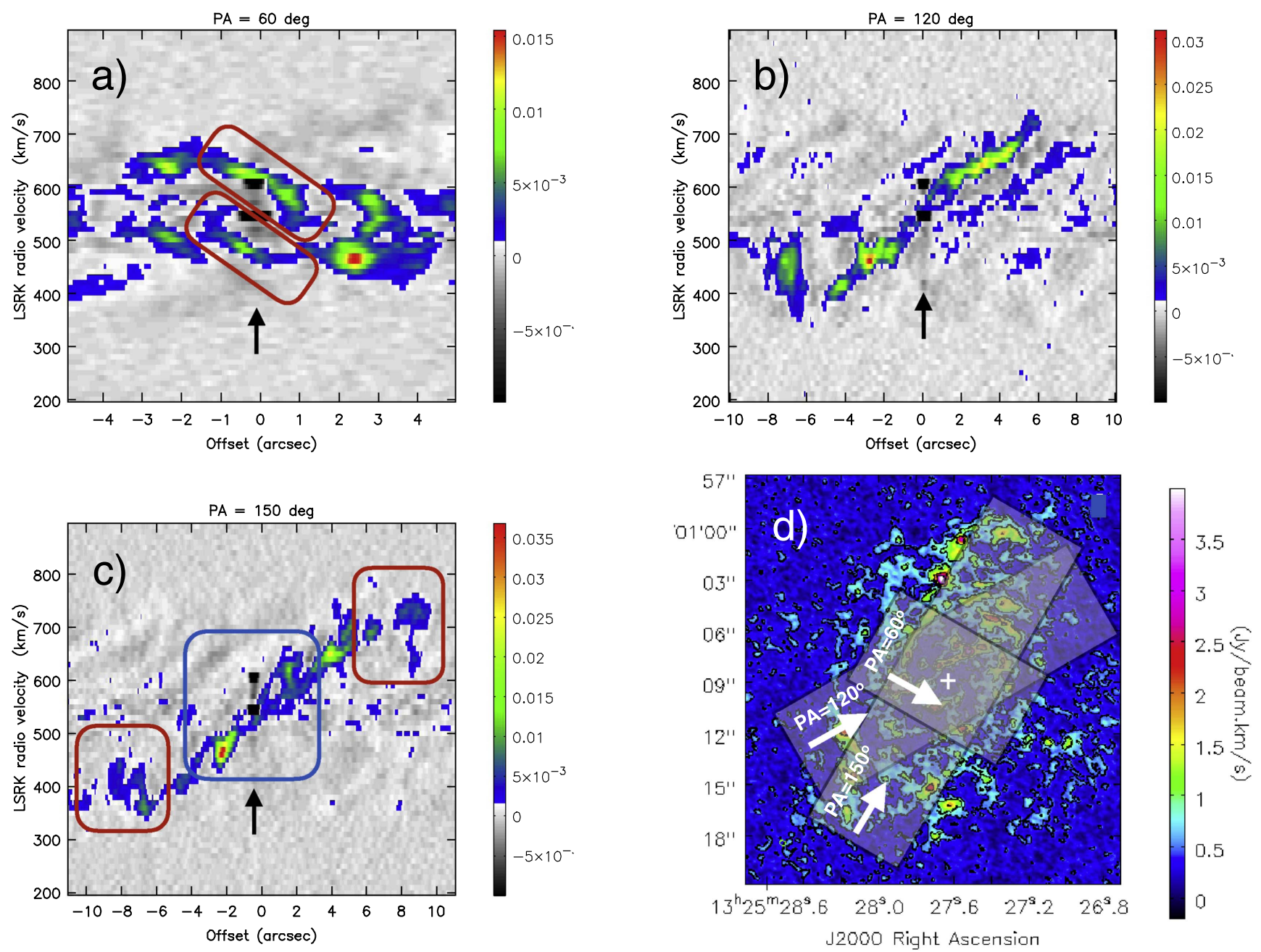

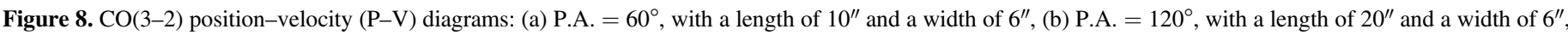

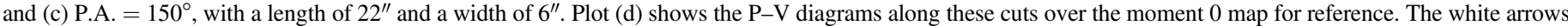

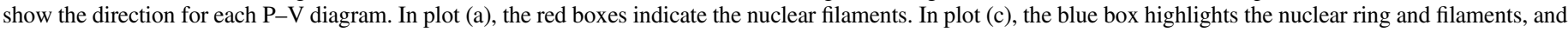
the red box the edges of the CND. The black arrow symbols indicate the AGN position.

The $\mathrm{CO}(6-5) \mathrm{P}-\mathrm{V}$ diagrams are displayed in Figure 13: (a) a P.A. $=115^{\circ}$ cut along the SE filament with a a length of 3"!5 and a width of $1 . \prime 6$, (b) a P.A. $=115^{\circ}$ cut along the NW filament with a length of $3 ! \prime 7$ and a width of $1 ! 9$, and (c) a P. A. $=115^{\circ}$ cut with a length of $9^{\prime \prime}$ and a width of $4^{\prime \prime}$, containing this time both nuclear filaments as well as the connecting points to the E and W of the AGN. The area probed in Figure 13(c) is essentially equivalent to that of the inner $9^{\prime \prime}$ of the $\mathrm{CO}(3-2)$ P-V diagram in Figure 8(c) (P.A. $=120^{\circ}$ ), and basically they are in agreement.

Figure 14 shows a zoom of the $\mathrm{P}-\mathrm{V}$ diagram of $\mathrm{CO}(6-5)$ at P.A. $=115^{\circ}$ of the NW filament. Along this filament, and farther outside from the nucleus, there is a plateau in the $\mathrm{P}-\mathrm{V}$ diagram of about $20 \mathrm{pc}$ in length. As indicated in Section 3.2, closer to the nucleus, the velocity gradient becomes steeper, $\Delta V / \Delta r=3.4 \mathrm{~km} \mathrm{~s}^{-1} \mathrm{pc}^{-1}$. A similar plateau and velocity gradient is also seen in the SE nuclear filament. At the closest distance from the AGN (1"!2, or $22 \mathrm{pc})$ along the NW nuclear filament, the P-V diagram may show a second plateau in velocity, and there is a signature of an even steeper velocity gradient of $\Delta V / \Delta r \simeq 12 \mathrm{~km} \mathrm{~s}^{-1} \mathrm{pc}^{-1}$ at about $0 . " 5$ from it, from 570 to $520 \mathrm{~km} \mathrm{~s}^{-1}$.

\section{4. $\mathrm{CO}(6-5) / C O(3-2)$ Line Ratios}

The difference in the critical densities of the $J=3-2$ and 6-5 CO molecular transitions is more than two orders of magnitude (see Section 1), and they are located at $T=33$ and $166 \mathrm{~K}$ above the ground state, respectively. In this subsection, we calculate $\mathrm{CO}(6-5) / \mathrm{CO}(3-2)$ line ratios to find out if there is any large gradient in the physical conditions of the molecular gas.

In order to reduce the effect of the different angular scales that are recovered by the $\mathrm{CO}(3-2)$ and $\mathrm{CO}(6-5)$ observations, we first performed imaging in these two data sets using CASA selecting visibilities for uv distances larger than $40 \mathrm{k} \lambda$ in task CLEAN keyword uvrange, i.e., the minimum uv distance in the $\mathrm{CO}(6-5)$ observations. The resulting maximum recoverable scales of the two data sets are similar and equal to $3^{\prime \prime}$ (or $54 \mathrm{pc}$ ). We then obtained primary beam-corrected maps and convolved the two images to the same resolution of 0.13 (or $5 \mathrm{pc}$ ) with a Gaussian kernel using CASA task IMSMOOTH. Absolute flux calibration uncertainties are as explained in Section 2.

$\mathrm{CO}(6-5)$ to $\mathrm{CO}(3-2)$ line ratios, $R_{65 / 32}$, for regions where $\mathrm{CO}(6-5)$ and $\mathrm{CO}(3-2)$ moment 0 maps exceeded $3 \sigma$, are shown in Figure 15. $R_{65 / 32}$ spans from 0.2 to 1.4 using main 

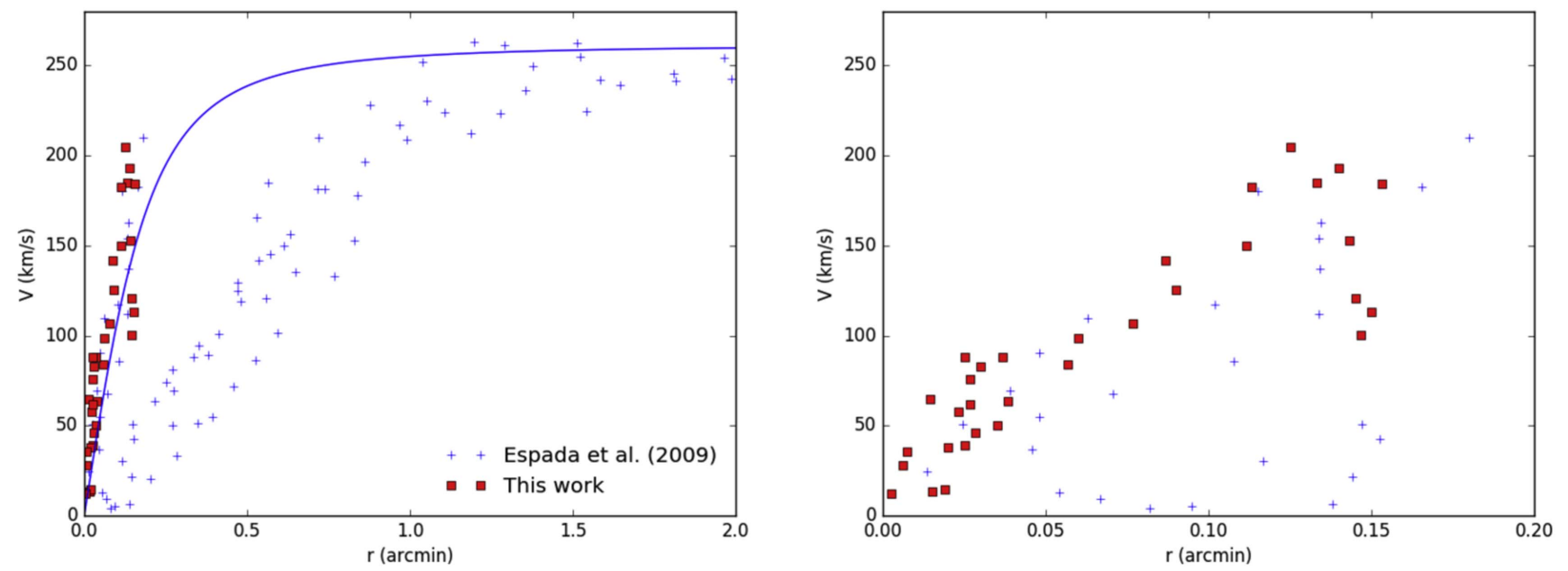

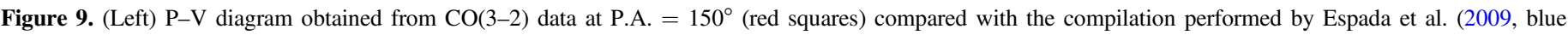

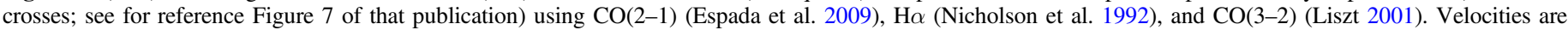

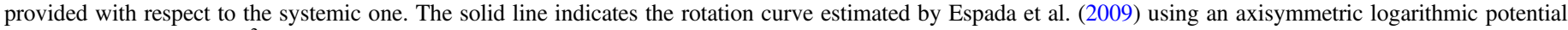
$\Phi_{0}(r)=0.5 \times \log \left(a+r^{2} / b\right)$ with $a=1.0$ and $b=0.05$. (Right) Same as the left panel but zooming into that portion of the plot corresponding to the CND.

brightness $T_{\mathrm{mb}}$ units (or 0.9-5.9 using flux units), with a mean value of 0.9 (respectively, 3.5). Along the two inner filaments $R_{65 / 32}$ (i.e., $\mathrm{NW}$ and $\mathrm{SE}$ ) there is a gradual increase toward the AGN by a factor of $2-3$. It is closest to the nucleus where the largest values are found and where velocity dispersions were also substantially larger (i.e., close to $20 \mathrm{~km} \mathrm{~s}^{-1}$ ).

Although unresolved, the average $R_{65 / 32}$ over the whole CND can also be inferred from the analysis by Israel et al. (2014). Obtaining integrated line intensities with Gaussian fits, and assuming the ratio normalized to a response of a $22^{\prime \prime}$ beam and corrections by absorption line loss, $R_{65 / 32}=0.4$ (or $\sim 1.85$ in flux units). This is a factor of two lower than the average over all regions in our $R_{65 / 32}$ map. It is unlikely that the different factor found in interferometric and single-dish experiments is due to flux loss effects. For further discussion, please refer to Section 7.

\section{5. $\mathrm{HCO}^{+}(4-3)$ and $\mathrm{HCN}(4-3)$ Emission and

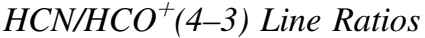

In Figures 16 and 17, we show the channel maps of $\mathrm{HCO}^{+}(4-3)$ and $\mathrm{HCN}(4-3)$ covering the inner $12^{\prime \prime}$ (same size as the $\mathrm{CO}(3-2)$ and $\mathrm{CO}(6-5)$ channel maps in Figures 6 and 10 for comparison) and the velocity interval from 415 to $695 \mathrm{~km} \mathrm{~s}^{-1}$ and from 415 to $615 \mathrm{~km} \mathrm{~s}^{-1}$ in $20 \mathrm{~km} \mathrm{~s}^{-1}$ bins, respectively. Due to limitations in the spectral setup, the HCN (4-3) line was only partially covered in our observations so the interval is cut beyond $615 \mathrm{~km} \mathrm{~s}^{-1}$. Although the field of view for these two transitions is almost identical to that of $\mathrm{CO}(3-2)$, namely $16^{\prime \prime}$, we did not detect any emission in the region outside $12^{\prime \prime}$ from the center. Similarly to the $\mathrm{CO}(6-5)$ transition, the blueshifted emission in these two dense gas tracers starts to the SE at the $435 \mathrm{~km} \mathrm{~s}^{-1}$ channel, and then continues to the redshifted side to the $\mathrm{NW}$, ending by the $635 \mathrm{~km} \mathrm{~s}^{-1}$ channel. Essentially, the $\mathrm{HCO}^{+}(4-3)$ line is detected along the nuclear filaments. $\mathrm{HCN}(4-3)$ is detected or tentatively detected (above $3 \sigma$ and in several channels) in just four regions and in all of them $\mathrm{HCO}^{+}(4-3)$ is also detected. For a similar rms, $\sigma=1.34$ mJy beam ${ }^{-1}, \mathrm{HCO}^{+}(4-3)$ emission is found to have a higher $\mathrm{S} / \mathrm{N}$ than the $\mathrm{HCN}(4-3)$ line. Note that the data of these two lines were taken simultaneously in two different spectral windows and calibrated in an identical manner, so the difference in amplitude cannot be explained in principle by absolute flux calibration uncertainties.

The average $\mathrm{HCN}(4-3)$ to $\mathrm{HCO}^{+}(4-3)$ intensity ratio in our maps is $R_{\mathrm{HCN} / \mathrm{HCO}^{+}} \simeq 0.5$, but there are regions where the ratio is even lower, e.g., $R_{\mathrm{HCN} / \mathrm{HCO}^{+}} \simeq 0.3$. To illustrate the low $R_{\mathrm{HCN} / \mathrm{HCO}^{+}}$, we present in Figure $18 \mathrm{HCO}^{+}(4-3)$ and $\mathrm{HCN}$ (4-3) spectra toward four different positions as indicated in the channel maps. The positions are (1) R.A. $=13^{\mathrm{h}} 25^{\mathrm{m}} 27^{\mathrm{s}} .905$, decl. $=-43^{\circ} 01^{\prime} 09$ !' $286 \quad(58 \mathrm{pc}$ from the nucleus $)$ (2) R.A. $=13^{\mathrm{h}} 25^{\mathrm{m}} 27^{\mathrm{s}} 68$, decl. $=-43^{\circ} 01^{\prime} 07$ !' 952 (20 pc from the nucleus), (3) R.A. $=13^{\mathrm{h}} 25^{\mathrm{m}} 27^{\mathrm{s}} .664$, decl. $=-43^{\circ} 01^{\prime} 10^{\prime \prime} .473$ (32 pc from the nucleus), and (4) R.A. $=13^{\mathrm{h}} 25^{\mathrm{m}} 27^{\mathrm{s}} \cdot 615$, decl. $=$ $-43^{\circ} 01^{\prime} 08^{\prime \prime} 805$ (center, in absorption).

We fitted single Gaussian profiles to the detected $\mathrm{HCN}(4-3)$ and $\mathrm{HCO}^{+}(4-3)$ lines, where the central velocity and width of the $\mathrm{HCN}(4-3)$ profile was fixed to that measured toward the brighter $\mathrm{HCO}^{+}(4-3)$. The fit parameters are given in Table 3 . The ratios for the emission lines are $R_{\mathrm{HCN} / \mathrm{HCO}^{+}}=0.37 \pm 0.08$ in position $1, R_{\mathrm{HCN} / \mathrm{HCO}^{+}}=0.40 \pm 0.1$ in position 2 , and $R_{\mathrm{HCN} / \mathrm{HCO}^{+}}<0.56$ in position 3 . For the absorption lines toward the AGN, the ratio is $R_{\mathrm{HCN} / \mathrm{HCO}^{+}}=0.2$ but note that it is likely material far from the center and just seen in projection (e.g., Espada et al. 2010).

High $R_{\mathrm{HCN} / \mathrm{HCO}^{+}}$ratios are often claimed to distinguish AGN over starburst conditions (e.g., Kohno et al. 2001; Krips et al. 2008; Izumi et al. 2016), but this does not hold for the nuclear regions of Cen A even though it is a well-known AGN. This is further discussed in Section 7.

\subsection{Molecular Gas Components within the CND}

Based on all the maps for the different transitions presented in Section 3, we enumerate next all the distinct and main molecular gas components that we can discern from large to small scales as we go closer to the center of Cen A. Figure 4 shows a simplified scheme that illustrates these main molecular components and the definitions we use in this paper. A summary of the properties of each component is provided in Table 2 and described next.

(1) CND: The major and minor axes of the CND are confirmed to be $20^{\prime \prime} \times 10^{\prime \prime}$, or $360 \mathrm{pc} \times 180 \mathrm{pc}$ in linear scale (without taking into account any projection effect). The 
Table 2

Derived Parameters of the Different Regions Detected in $\mathrm{CO}(3-2)$ and $\mathrm{CO}(6-5)$

\begin{tabular}{|c|c|c|c|c|c|}
\hline Component & $\begin{array}{c}\text { Peak }^{\mathrm{a}} \\
\left(\mathrm{Jy} \mathrm{beam}^{-1} \mathrm{~km} \mathrm{~s}^{-1}\right)\end{array}$ & $\begin{array}{l}\text { Velocity range } \\
\left(\mathrm{km} \mathrm{s}^{-1}\right)\end{array}$ & $\begin{array}{l}S_{12} \mathrm{CO}(3-2) \\
\left(\mathrm{Jy} \mathrm{km} \mathrm{s}^{-1}\right)\end{array}$ & $\begin{array}{l}S_{12} \mathrm{CO}(6-5)^{\mathrm{b}} \\
\left(\mathrm{Jy} \mathrm{km} \mathrm{s}^{-1}\right)\end{array}$ & $\begin{array}{c}M_{\mathrm{gas}}{ }^{\mathrm{c}} \\
\left(10^{6} M_{\odot}\right)\end{array}$ \\
\hline Filament NW & $3.7 \pm 0.2 / 5.9 \pm 0.8$ & $550-660$ & $45 \pm 1(46)$ & $151 \pm 6(176)$ & 1.42 \\
\hline Filament SE & $2.4 \pm 0.2 / 4.4 \pm 0.8$ & $410-570$ & $23 \pm 1(23)$ & $93 \pm 5(106)$ & 0.71 \\
\hline Nuclear ring ${ }^{\mathrm{d}}$ & $6.8 \pm 0.2 / 8.4 \pm 0.8$ & $420-700$ & $339 \pm 3(364)$ & $888 \pm 20(1259)$ & 11.25 \\
\hline CND & $6.8 \pm 0.2 / 8.4 \pm 0.8$ & $290-820$ & $1102 \pm 6(1552)$ & $\ldots$ & 47.95 \\
\hline
\end{tabular}

Notes.

${ }^{a}$ Peak flux densities are derived from the primary beam-corrected maps. Two values are given, the first referring to $\mathrm{CO}(3-2)$ and the second to $\mathrm{CO}(6-5)$.

${ }^{b}$ The total fluxes for $\mathrm{CO}(3-2)$ and $\mathrm{CO}(6-5)$. Numbers in parentheses indicate primary beam-corrected values.

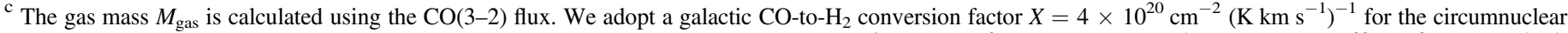

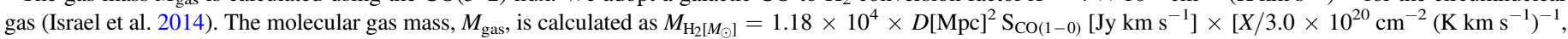

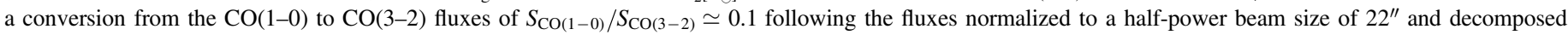
between the CND and extended component (Israel et al. 2014), and a factor of 1.36 to account for elements other than hydrogen (Cox 2000).

dValues for the nuclear ring contain $\mathrm{CO}(3-2)$ and $\mathrm{CO}(6-5)$ emission in the inner 8 ". 5 .

position angle is P.A. $=155^{\circ}$. This is in good agreement with the CND size and orientation provided by Espada et al. (2009), considering the coarser angular resolution of $6^{\prime \prime}$. The inclination of the disk was estimated to be $i \simeq 70^{\circ}$, but with our new values for the major and minor axes, the inclination assuming a circular disk is slightly smaller, $i \simeq 60^{\circ}$. It is composed of multiple filamentary and clumpy structures, possibly arm-like features or streamers. In Figure 4 we indicated as black lines most of the filaments that can be found within the CND as seen from our maps. The velocity width of the CND is $\Delta V \simeq 475 \pm 7 \mathrm{~km} \mathrm{~s}^{-1}$ (FWZI), without correcting for inclination. With the orientation and the kinematics of the CND, the several $10-100 \mathrm{pc}$ scale filamentary structures within the CND are likely trailing. In the external parts of this CND $\left(r>8^{\prime \prime}\right.$, or $144 \mathrm{pc}$, from the center), the molecular gas has large velocity widths of $10 \mathrm{~km} \mathrm{~s}^{-1}$ and up to $40 \mathrm{~km} \mathrm{~s}^{-1}$. These regions correspond to the high-velocity wings of the $\mathrm{CO}(3-2)$ spectra. A double-peaked distribution is present in both $\mathrm{CO}(3-2)$ and $\mathrm{CO}(6-5)$ spectra, although it is clearer in the latter.

(2) Nuclear ring: Deeper inside the CND, there is a ring-like feature with a major and minor axis of $9^{\prime \prime} \times 6^{\prime \prime}$ $(162 \mathrm{pc} \times 108 \mathrm{pc})$, and also at P.A. $=155^{\circ}$, which is formed by multiple filaments or streamers leading to it. If the ring structure is coplanar and has a circular shape, then the inclination would be $i \simeq 50^{\circ}$. This ring-like structure has a velocity width of $\sim 260 \mathrm{~km} \mathrm{~s}^{-1}$. This component is detected in $\mathrm{CO}(3-2)$ as well as partially in $\mathrm{CO}(6-5)$.

(3) Nuclear filaments: There are two nearly parallel filamentary structures to the SE and NW of the AGN of about $2^{\prime \prime}$ in length (or $\sim 40 \mathrm{pc}$ ) contained within the nuclear-ring-like structure, with P.A. $\simeq 120^{\circ}$, and with a rotational symmetry of $180^{\circ}$ around the AGN. These components are most prominent in $\mathrm{CO}(3-2), \mathrm{CO}(6-5)$, and $\mathrm{HCO}^{+}(4-3)$, and just partially detected in $\mathrm{HCN}(4-3)$. The NW filament is aligned from R.A., decl. [J2000] $=13^{\mathrm{h}} 25^{\prime} 2$ ? $^{\prime \prime} 692$, $-43^{\circ} 01^{\prime} 08^{\prime \prime} 19$ to $13^{\mathrm{h}} 25^{\prime} 27^{\prime \prime} 407,-43^{\circ} 01^{\prime} 06^{\prime \prime} 833$, and from $V=555$ to $695 \mathrm{~km} \mathrm{~s}^{-1}$, and the SE filament from R.A., decl. = $13^{\mathrm{h}} 25^{\prime} 27^{\prime \prime} .536,-43^{\circ} 01^{\prime} 09^{\prime \prime} .65$ to $13^{\mathrm{h}} 25: 27$ !' $772,-43^{\circ} 01^{\prime}$ 10 " 882 , and from $V=455$ to $555 \mathrm{~km} \mathrm{~s}^{-1}$. There is an increase in velocity of $\sim 50 \mathrm{~km} \mathrm{~s}^{-1}$ with respect to that of the overall CND. There is an asymmetry between these two filaments. The filament to the NW is a factor of two brighter than the component to the SE. Although both filamentary structures are symmetric with respect to the center of the galaxy, the SE nuclear filament is curved toward the $\mathrm{N}$ as it extends away from the AGN and connects it to the nuclear ring structure with $9^{\prime \prime}$ diameter. Despite the asymmetry, if we add all the flux from the component to the $\mathrm{N}$ and $\mathrm{W}$ of the AGN and to the $\mathrm{S}$ and $\mathrm{E}$ in the $\mathrm{CO}(6-5)$ map, for example, we find that the total fluxes are quite equal: $186 \mathrm{Jy} \mathrm{km} \mathrm{s}^{-1}$ versus $180 \mathrm{Jy} \mathrm{km} \mathrm{s}^{-1}$. There are well-defined molecular clumps in these two structures. In the transitions reported in this paper, one can see around four to six clumps on each side.

The connecting point between the SE nuclear filament and ring is the brightest in all probed transitions of all the components presented in this paper, and also has a large velocity dispersion of $\sim 20 \mathrm{~km} \mathrm{~s}^{-1}$. A similar connecting point, but with a slightly slower dispersion and not as prominent as the previous one, can be found at the opposite side (i.e., to the $\mathrm{W}$ ). These connecting points are also linked to additional filaments that are nearly perpendicular to the nuclear filaments and form part of the nuclear ring.

(4) Nuclear disk: In Section 1 we introduced the few tens of parsec-scale-sized nuclear disk, well inside the area covered by the nuclear filaments, which contains ionized and molecular gas that presumably is the fuel feeding the nuclear massive object. While ionized gas shows distributions elongated along the jet and is likely related to it, very warm (typically 1000-2000 K) molecular hydrogen as traced by $\mathrm{H}_{2}(J=1-0) \mathrm{S}(1)(2.122 \mu \mathrm{m})$ using VLT/ SINFONI in the inner $3^{\prime \prime}(\sim 50 \mathrm{pc})$ follows a rotating nuclear disk (Neumayer et al. 2007), although the distribution shows that this disk might be quite irregular. Part of the gas in the nuclear $\mathrm{H}_{2}$ disk may have been impacted by the jet (Bicknell et al. 2013) and excited by shocks (Israel et al. 2017).

Figure 19 shows the comparison of the channel maps of the $\mathrm{CO}(6-5)$ and the $\mathrm{H}_{2}$ lines. Although most of the warm and dense gas close to the nucleus $(r<20 \mathrm{pc})$ is not detected as traced by the transitions investigated in this paper, we observe that the endings of the two nuclear filaments and a third weaker component approaching the nucleus from the E of the AGN (clearly seen in the $\mathrm{CO}(3-2)$ and $\mathrm{CO}(6-5)$ lower contours in the channel maps) are counterparts of the molecular gas traced by the $\mathrm{H}_{2}$ line within the nuclear disk. The $\mathrm{CO}$ filaments abruptly end in the probed transitions $\sim 20 \mathrm{pc}$ from the AGN, but the $\mathrm{H}_{2}$ maps show that these continue in a warmer gas phase, probably shock excited, and then wind up in the form of nuclear spiral arms (see also Figure 1(b)). Although with sizes a factor of 10 larger, these filaments/arms are 


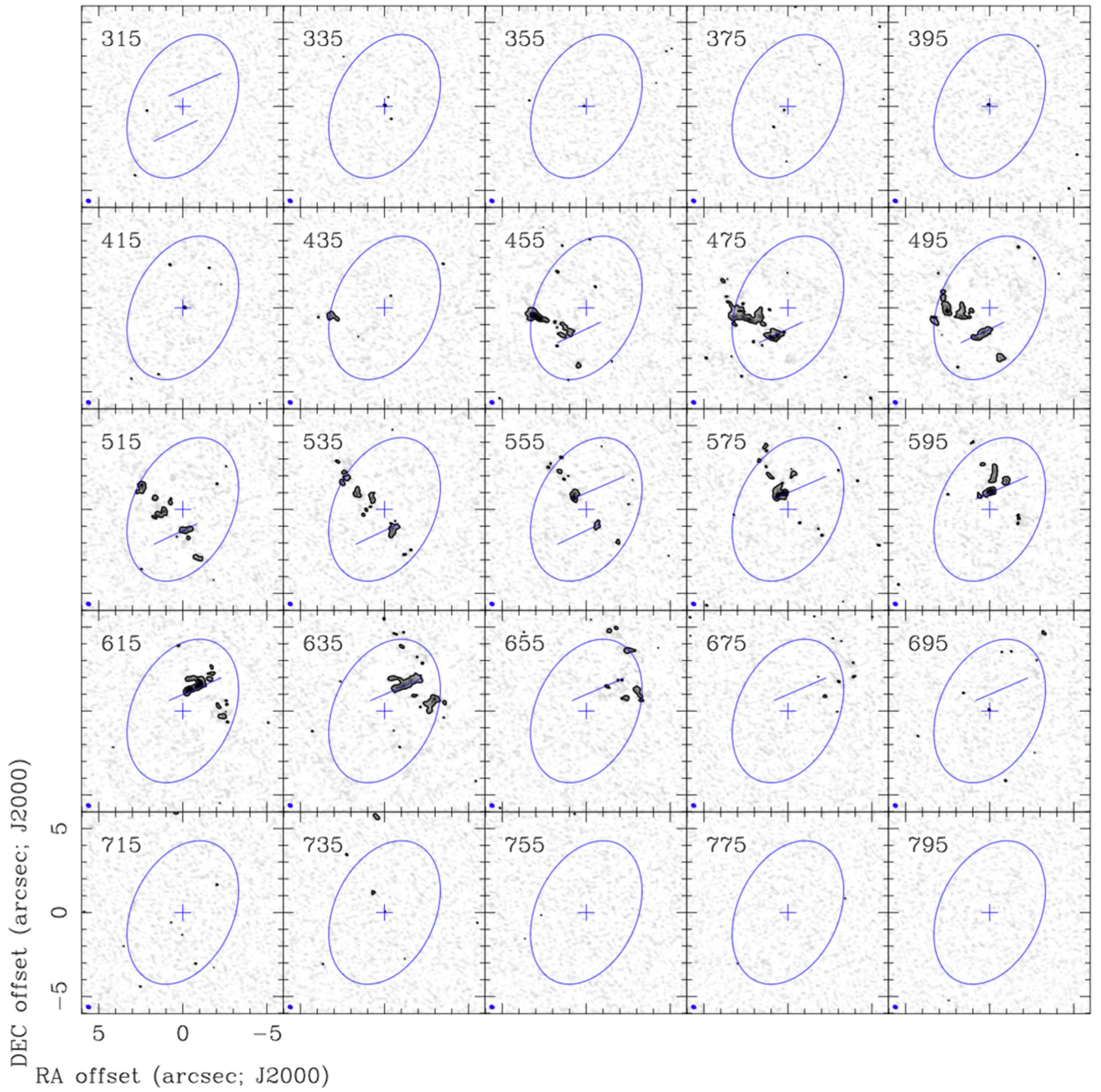

Figure 10. $\mathrm{CO}(6-5)$ channel maps of the CND of Cen A in the LSR velocity range $V=315-795 \mathrm{~km} \mathrm{~s}^{-1}$ in $20 \mathrm{~km} \mathrm{~s}^{-1}$ bins. The size of the maps is $12^{\prime \prime}$. The velocities are shown in the upper-left corner, and the synthesized beam at the bottom-left corner of each panel. The rms noise of individual channels is $4.1 \mathrm{mJy}$ beam $^{-1}$. The contour levels are $5 \sigma$ and $25 \sigma$. The cross sign shows the position of the AGN: R.A. $=13^{\mathrm{h}} 25^{\mathrm{m}} 27^{\mathrm{s}} .615$, decl. $=-43^{\circ} 01^{\prime} 08^{\prime \prime}$. 805 . See Figure 4 for a description of the symbols representing the main molecular components of the CND of Cen A.

reminiscent of the Galactic Center, where molecular and ionized components (circumnuclear disk and mini spirals) are seen within the inner few parsecs of Sgr A* (e.g., Ekers et al. 1983; Lo \& Claussen 1983; Zhao et al. 2009; Martín et al. 2012; Tsuboi et al. 2016).

Overall, these nuclear spiral features within the nuclear disk of Cen A create a distribution that remind us of another structure that resembles a ring-like feature, but this time at $r=0$ ". $5(10 \mathrm{pc})$. Farther inside this $10 \mathrm{pc}$ ring-like feature, two additional $180^{\circ}$ rotationally symmetric regions are found along the $\mathrm{N}-\mathrm{S}$ direction of about $10 \mathrm{pc}$ in length and with even larger velocity dispersions $\left(\sim 200 \mathrm{~km} \mathrm{~s}^{-1}\right.$ from $\left.\mathrm{H}_{2}\right)$ than anywhere else within the CND as probed in our CO maps.

\subsection{Molecular Gas Mass}

We calculate in this subsection the mass of the different molecular gas components found in our maps. We use a conversion factor between the integrated $\mathrm{CO}$ intensity and $\mathrm{H}_{2}$ column density $X=N_{\mathrm{H}_{2}} / I_{\mathrm{CO}}=4 \times 10^{20} \mathrm{~cm}^{-2}\left(\mathrm{~K} \mathrm{~km} \mathrm{~s}^{-1}\right)^{-1}$ for the CND (Israel et al. 2014), with an uncertainty of a factor of two. This value is a factor of 10 larger than that observed in other nuclear regions of galaxies (Maloney \& Black 1988; Wilson 1995; Mauersberger et al. 1996; Weiß et al. 2001) and that previously used by Espada et al. (2009) to calculate the molecular gas mass of the CND. The masses derived here should be rescaled if a better factor for Cen A is obtained. We 


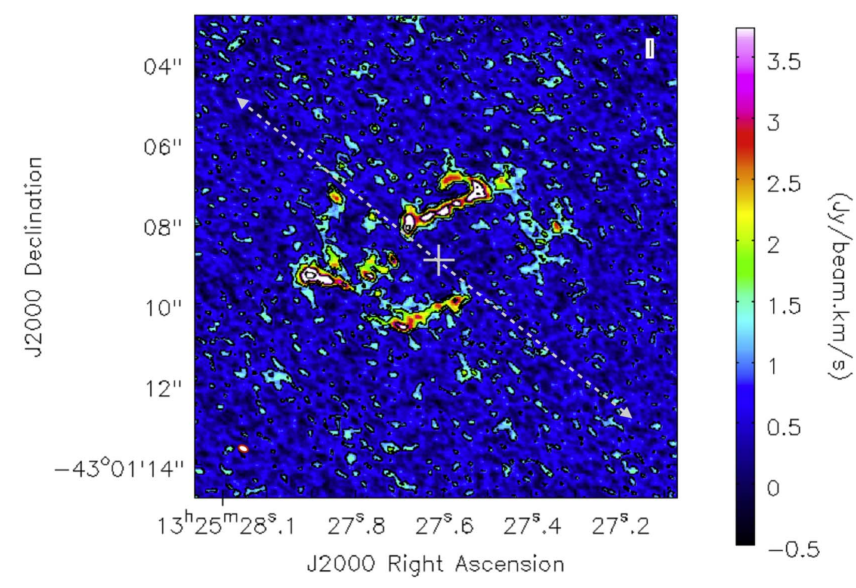

Figure 11. $C O(6-5)$ integrated intensity map of the CND of Cen $A$, with the color scale ranging from -0.5 to $3.6 \mathrm{Jy} \mathrm{beam}^{-1} \mathrm{~km} \mathrm{~s}^{-1}$. The size of the map is $12^{\prime \prime}$, and the HPBW is $8^{\prime \prime}$. Contour levels are at 1.614, 3.2, and

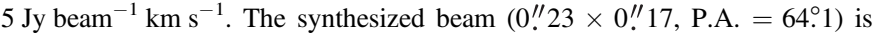
indicated by an ellipse at the bottom-left corner of the plot. The cross sign shows the position of the AGN: R.A. $=13^{\mathrm{h}} 25^{\mathrm{m}} 27^{\mathrm{s}} \cdot 615$, decl. $=-43^{\circ} 01^{\prime}$ 08 . 805 . The dashed line indicates the jet direction at P.A. $=51^{\circ}$.

also use a line ratio between ${ }^{12} \mathrm{CO}(1-0)$ and (3-2) fluxes of 0.1 over the whole CND, again following Israel et al. (2014).

The gas mass obtained from the $\mathrm{CO}(3-2)$ map is $M_{\text {gas }} \simeq 4.8 \times 10^{7} M_{\odot}$. Flux loss was measured in Section 3.2 to be $\sim 50 \%$, so the final estimate is $M_{\text {gas }} \simeq 9 \times 10^{7} M_{\odot}$. This is consistent with the measurement $M_{\mathrm{gas}} \simeq 8 \times 10^{7} M_{\odot}$ inside a projected distance of $r<200 \mathrm{pc}\left(12^{\prime \prime}\right)$ in Espada et al. (2009) if we apply the $X$ factor given by Israel et al. (2014). This is also in agreement with the mass of the circumnuclear gas using single-dish measurements for many $\mathrm{CO}$ transitions and an LVG analysis, which yielded $M_{\text {gas }}=8.4 \times 10^{7} M_{\odot}$, also assuming a $35 \%$ mass contribution by helium (Israel et al. 2014).

Table 2 exhibits the derived main parameters of the circumnuclear gas disk, nuclear ring, and nuclear filaments (NE and $\mathrm{SW}$ ) in $\mathrm{CO}(3-2)$ and $\mathrm{CO}(6-5)$. These parameters include peak flux densities, velocity ranges, total $\mathrm{CO}(3-2)$ and $\mathrm{CO}(6-5)$ fluxes, and the corresponding molecular gas masses by using the $\mathrm{CO}(3-2)$ fluxes.

\section{Warped Disk or Non-circular Motions? Mechanisms Feeding the AGN from Kiloparsec to Parsec Scales}

\subsection{A Warped Disk?}

In the past, a warped and thin disk model had been used to reproduce the observations both at a large scale (e.g., Quillen et al. 2006) as well as at a few parsec scale using the $\mathrm{H}_{2}$ line (Neumayer et al. 2007). Neumayer et al. (2007) show by applying the Kinemetry analysis (Krajnović et al. 2006) to the velocity field map of the nuclear disk that it is characterized by a mean inclination angle of $i=45^{\circ}$ and a P.A. $=155^{\circ}$ assuming a warped disk model to describe its gas kinematics. Quillen et al. (2006) used the warped disk model to reproduce the morphology of the parallelogram feature seen in mid-IR (Spitzer), on top of previous studies. Quillen et al. (2010) compiled inclinations and position angles from the literature and identified that there might be (assuming that a warped disk model applies) three major changes of inclination and P.A. at about $1.3 \mathrm{kpc}(1 ! 2)$, at about $600 \mathrm{pc}\left(33^{\prime \prime}\right)$, and a third kink at a radius of about $100 \mathrm{pc}\left(5^{\prime \prime}\right)$. The field of view and angular resolution in the ALMA observations fill the missing gap from tens of parsec to the $r=200 \mathrm{pc}$ scale within the CND. The existence of the ring and the nuclear filaments are likely related to the third kink.

We also fitted the $\mathrm{CO}(3-2)$ velocity field using Kinemetry. The Kinemetry method performs harmonic expansion of 2D maps such as surface brightness, velocity, or velocity dispersion, along the best-fitting ellipses in order to detect morphological and kinematic components. We use the fitting over the line-of-sight velocity distribution. If we assume circular orbits in a warped disk, then one can extract the inclination and position angle of each ring as a function of radius. We follow this approach to fulfill the following three aims: (1) to identify the general properties of the circumnuclear gas assuming a warped disk model is valid, (2) to compare the results with previous fits using the same assumption but for other linear scales, and (3) to quantify deviations from this assumption and identify regions that are significantly different.

Several kinematic parameter profiles as a function of radius were obtained from the $\mathrm{CO}(3-2)$ velocity field at a scale of 1 !"2 (Figure 20 and Table 4): (a) the kinematic P.A. or orientation of the maximum velocity, (b) the inclination (assuming circular orbits) obtained from the axial ratio $q$ (i.e., flattening of the ellipse, where $q=\cos i$ ), (c) $k 1$, the amplitude of bulk motions (rotation curve), and (d) $k 5 / k 1$, the ratio between harmonics $k 1$ and $k 5$, where $k 5$ is the higher-order term which is not fitted, and represents deviations from simple rotation and points to complex kinematical components. The plots show the trends in these parameters up to a radius of $10^{\prime \prime}$.

A ring width of 1 !" 2 was used and the following trends are present in the results. Note that other widths were also used, showing similar results. In the plots, values smaller than $3^{\prime \prime}$ are uncertain due to a lack of $\mathrm{CO}(3-2)$ emission there and are not presented. Within the inner $5^{\prime \prime}$, the distribution and kinematics appear peculiar. Overall, the mean kinematic P.A. is $145^{\circ}$, with values close to $120^{\circ}$ up to $5^{\prime \prime}$, likely due to the effect of the nuclear filaments. The kinematic P.A. is close but offset from the photometric P.A. of the disk, which was estimated to be $155^{\circ}$. Since the kinematic P.A. and the photometric P.A. of the disk are different, it indicates that the distribution/kinematics are not axisymmetric. The inclination varies from $58^{\circ}$ to $46^{\circ}$, assuming circular orbits. On average, the inclination within the CND is $50^{\circ}-60^{\circ}$ and agrees well with the inclination obtained for the CND and nuclear ring. $k 1$ is very high at small radii because of the two nuclear filaments, but it is $\sim 100 \mathrm{~km} \mathrm{~s}^{-1}$ within most of the disk. Above $8^{\prime \prime}$, it decreases considerably and it cannot be considered to be representative of the rotation speed. This is because of the large velocity dispersion component at the edge of the CND, and probably also because of the extended molecular gas emission located at large radii $(r>1 \mathrm{kpc})$ and seen in projection. $k 5 / k 1$ is very large $(\sim 0.4)$ above $9^{\prime \prime}$, partly due to multiple velocity components and the low values of $k 1$ as a result of some contamination with extended emission. The average from $2^{\prime \prime}$ to $9^{\prime \prime}$ is very large in comparison with other studies on nearby galaxies (e.g., Barth et al. 2016), where $k 5 / k 1$ is typically $\lesssim 0.05$. This indicates that there are large deviations from circular motion due to complex kinematics of the molecular gas and/or multiple components along the line of sight.

Figure 21 shows the inclinations and position angles as a function of radius compiled by Quillen et al. (2010), using data from Quillen et al. (2006), Neumayer et al. (2007), and Espada et al. (2009), as well as those presented in this paper for 

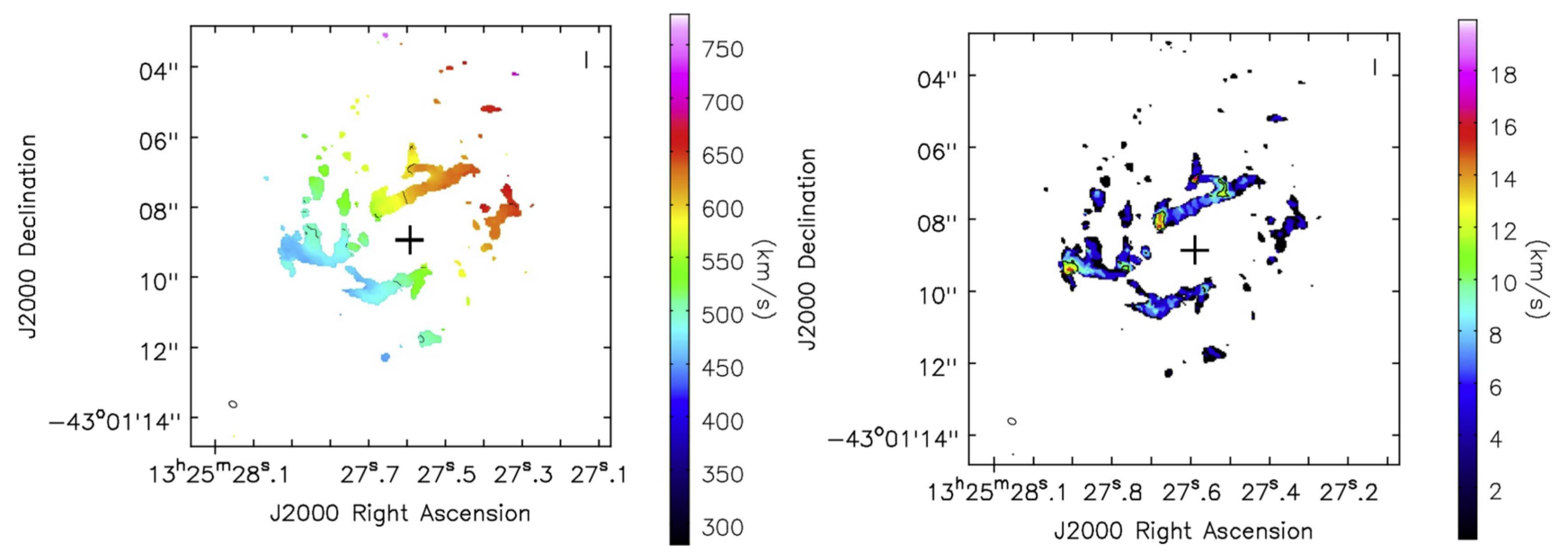

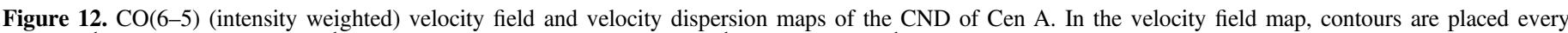

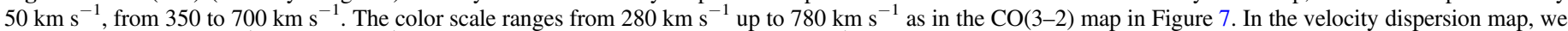

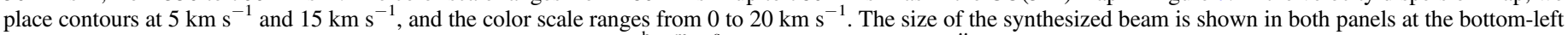
corner. The cross sign shows the position of the AGN: R.A. $=13^{\mathrm{h}} 25^{\mathrm{m}} 27^{\mathrm{s}} .615$, decl. $=-43^{\circ} 01^{\prime} 08^{\prime \prime} .805$.

comparison. The data presented here fills for the first time the gap between the several hundred to tens of parsec scale, and the obtained position angles and inclinations seem to naturally follow previously published results. Finally, in Figure 22 we plotted all ellipses fitted by Kinemetry from large to small scales. The panel on the left shows the fits for large scales, i.e., the inner $300^{\prime \prime}$, while the one on the right shows the small scales, i.e., the inner $16^{\prime \prime}$. The red ellipses in both panels correspond to the fits performed in this paper using the $\mathrm{CO}(3-2)$ data, and in blue the fits obtained by Espada et al. (2009) and Neumayer et al. (2007).

In summary, although we tried to fit the best warped disk model possible from kinematic information, deviations from simple rotation and complex kinematic components are apparent. Using this simple warped disk model, it would not be possible to reproduce the distribution. Therefore, we invoke non-circular motions as one of the main ingredients to explain the observed distribution and kinematics.

\subsection{Non-circular Motions}

In Section 3.6, we described the main molecular gas components that we could discern within the CND of Cen A. There are large deviations from axisymmetry in the distribution and kinematics, and non-circular motions are needed to explain the observations. We favor the scenario where non-circular motions play a major role due to the existence of well-aligned nuclear filaments and a nuclear ring.

Non-circular motions in the gas disk of Cen A had been invoked before, although at a larger scale. Espada et al. (2009) provided evidence that non-circular motions in the molecular gas may be present at least at kiloparsec scales. It was argued that the contribution of a weak non-axisymmetric potential (together with a warp as assumed in previous work) is able to reproduce the $\mathrm{CO}$ line distribution and kinematics. In particular, it could reproduce well the curved emission resembling spiral arms at kiloparsec scales (Espada et al. 2012), the formation of the CND, the connection of this CND to gas at larger radii, and the lack of emission along the E-W direction in projection within the parallelogram filaments imaged in the mid-infrared (Quillen et al. 2006). The latter is interpreted as a gap of emission at $200 \mathrm{pc}<r<800 \mathrm{pc}$
(Quillen et al. 2006). A possible gap of $\mathrm{CO}$ emission in the inner $r<80 \mathrm{pc}$ of the CND was suggested by Espada et al. (2009) and Espada (2013), which we now identify as the area inside the nuclear ring. The lack of high angular resolution and high sensitivity observations had prevented the identification of the nuclear filaments until now.

Since the gas is dissipative (i.e., it will shock at orbit crossings), the kinematic effects on the molecular gas are important when a non-axisymmetric potential is introduced and the resulting large non-radial hydrodynamical (pressure) forces can exert torques on the gas, which alter its orbital motion. The gas flows down the shocks and "sprays" back out to large radii, encountering another shock at the opposite region, and repeating the pattern, which makes bars important agents for the gas in spiral galaxies to lose part of its angular momentum and form substantial gas concentrations in their central regions (e.g., Athanassoula 1992; Sakamoto et al. 1999).

Additional mechanisms are invoked for driving gas past the inner Lindblad resonance to feed SMBHs that power AGNs and nuclear SBs. Shlosman et al. (1989, 1990) proposed the bars within bars model, in which a primary (mostly stellar) bar would lead gas to the center, where it would become unstable and form a gaseous bar, further depositing gas into a nuclear disk of tens of parsec scale. This inspired many theoretical and observational works. Friedli \& Martinet (1993) showed that bars within bars can form in 3D self-consistent simulations with stars and gas. The gaseous component was shown to be essential for the decoupling of the nuclear bar. The large-scale stellar bar can then be rapidly destroyed by the central mass concentration. Englmaier \& Shlosman (2004) investigated the mechanism of formation and dynamical decoupling of bars within bars, with gaseous nuclear bars encompassing the full size of the galactic disk and hosting a double inner Lindblad resonance. As these structures become massive and selfgravitating, the nuclear bars lose internal angular momentum to the primary bars, increase their strength, diminish the nuclear bar size, and increase the nuclear bar pattern speed. The viscosity of the gas is an important parameter for the decoupling of nested bars.

Observationally, approximately one-third of barred galaxies host a secondary nuclear bar in addition to the primary largescale stellar bar with pattern speeds exceeding those of the 

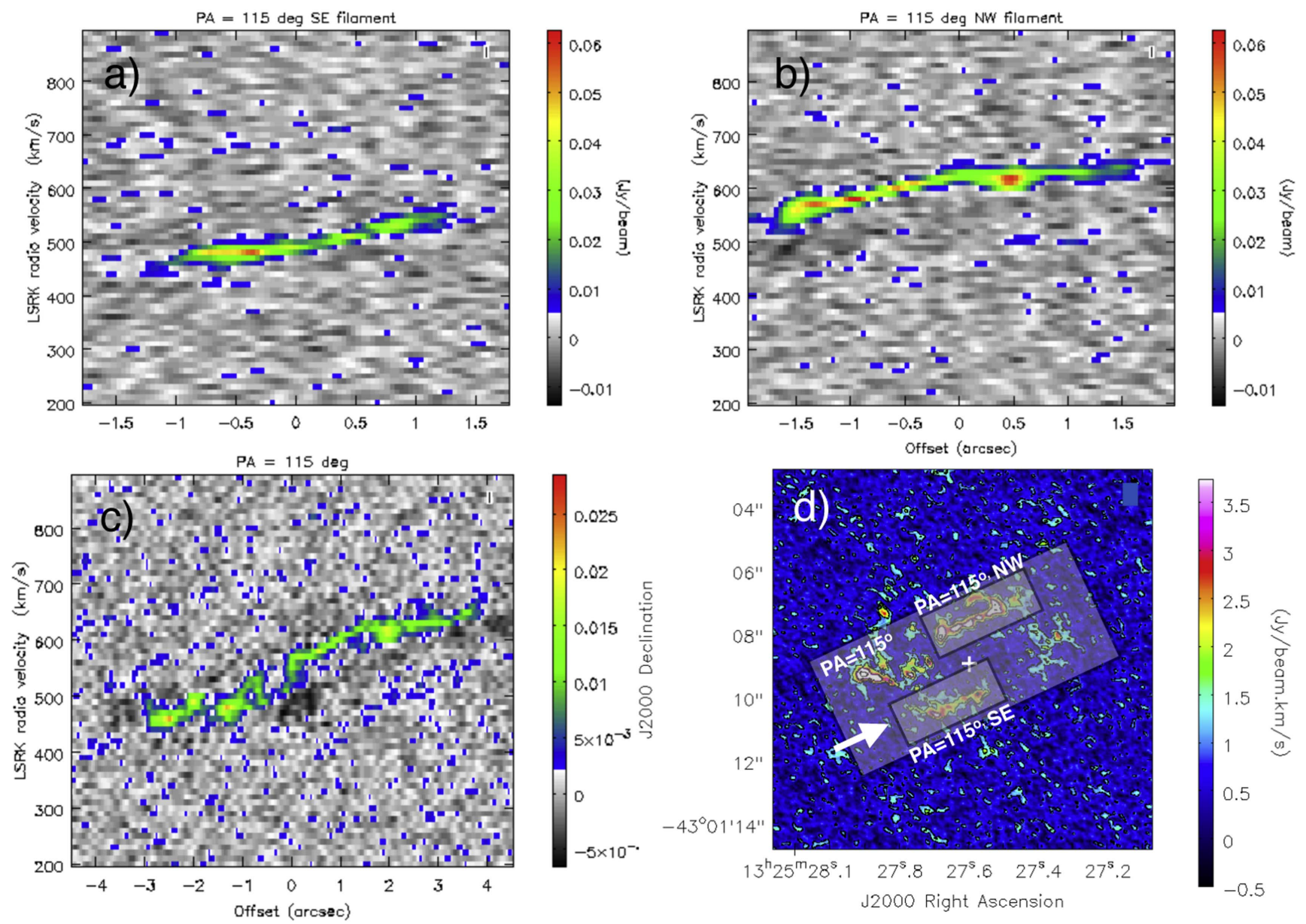

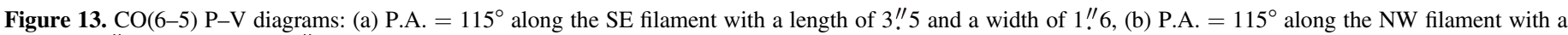

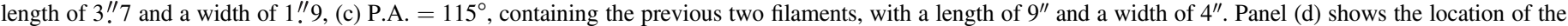
cuts of the P-V diagrams over the moment $0 \mathrm{CO}(6-5)$ map for reference. The (white) arrow symbol indicates the direction used in the P-V diagrams.

large-scale (primary) stellar bars (e.g., Pfenniger \& Norman 1990; Friedli \& Martinet 1993; Wozniak et al. 1995; Jungwiert et al. 1997; Erwin 2004, and references therein). An increasing number of publications show streaming gas flows down to about tens of parsecs from the nucleus of galaxies as a result of (stellar) nested bars (e.g., Fathi et al. 2006, 2013 for NGC 1097; Schinnerer et al. 2006, 2007 for NGC 6946; Meier et al. 2008 and Meier \& Turner 2012 for Maffei 2). A secondary bar in our Galaxy was reported by Alard (2001) and simulations to illustrate the putative nested bars were presented by Namekata et al. (2009). In the small inner bar models with sizes of $200 \mathrm{pc}$, straight shocks are formed within the inner bar, leading to a nuclear gas disk formed at the center with size of $15 \mathrm{pc}$ and mass of $\sim 10^{7} M_{\odot}$. All of these cases are suggested to be caused by a nuclear stellar bar. Moreover, all nested bar observations and simulations so far have focused on spiral galaxies, but not on elliptical galaxies whose ISM is replenished by external gas.

The inner ring and nuclear filaments seen in Cen A with the help of the ALMA data and VLT/SINFONI data are likely due to non-circular motions. In this scenario, these nuclear filaments are the loci of shocks. This is reasonable because of the peculiar observed distribution of the $\mathrm{CO}$ and $\mathrm{H}_{2}$ nuclear filaments, i.e., they are nearly straight and are characterized by a $180^{\circ}$ rotational symmetry. This is further reinforced by the distinct kinematics of the nuclear filaments as revealed from the
P-V diagrams. Figure 8(c) shows a clearly steeper slope in the $\mathrm{P}-\mathrm{V}$ diagram in both nuclear filaments, and Figure 8(a) (also Figures 13 and 14 for $\mathrm{CO}(6-5)$ ) shows that the $\mathrm{P}-\mathrm{V}$ curves of the nuclear filaments systematically avoid the location of the AGN and $V_{\text {sys }}$, therefore these components are likely at forbidden velocities (Combes 1991). Overall, the distribution and kinematics are in agreement with existing simulations of gas under barred potentials causing non-circular motions (e.g., Wada et al. 1994; Namekata et al. 2009). As for the nuclear disk, there is further evidence that the $\mathrm{H}_{2}$ and ionized line emission of the Cen A center is likely dominated by shocks (Israel et al. 2017 and references therein). Based on $\mathrm{H}_{2}$ line ratios, the warm molecular gas is likely excited by modest shock velocities of 5-20 $\mathrm{km} \mathrm{s}^{-1}$ (Ogle et al. 2010; Israel et al. 2017), which might be partly explained by non-circular motions.

Within the CND, significant self-gravitation $\left(M_{\mathrm{gas}}>0.3 M_{\mathrm{dyn}}\right)$ would be required to trigger a gaseous bar instability, but under these conditions, due to star-forming gas, self-gravity would decrease quickly and a very short lifetime of the gaseous bar would result (Friedli 1998). In Section 3.7, we estimated the molecular gas mass enclosed within the CND as $M_{\text {gas }} \simeq 9 \times 10^{7} M_{\odot}$. The velocity dispersion can be assumed to be small with respect to the orbital velocity within the extent of the CND, and then the dynamical mass at a radius $r$ is $M_{\text {dyn }}=V^{2} r / G$, where $V$ is the rotation velocity and $G$ is the 


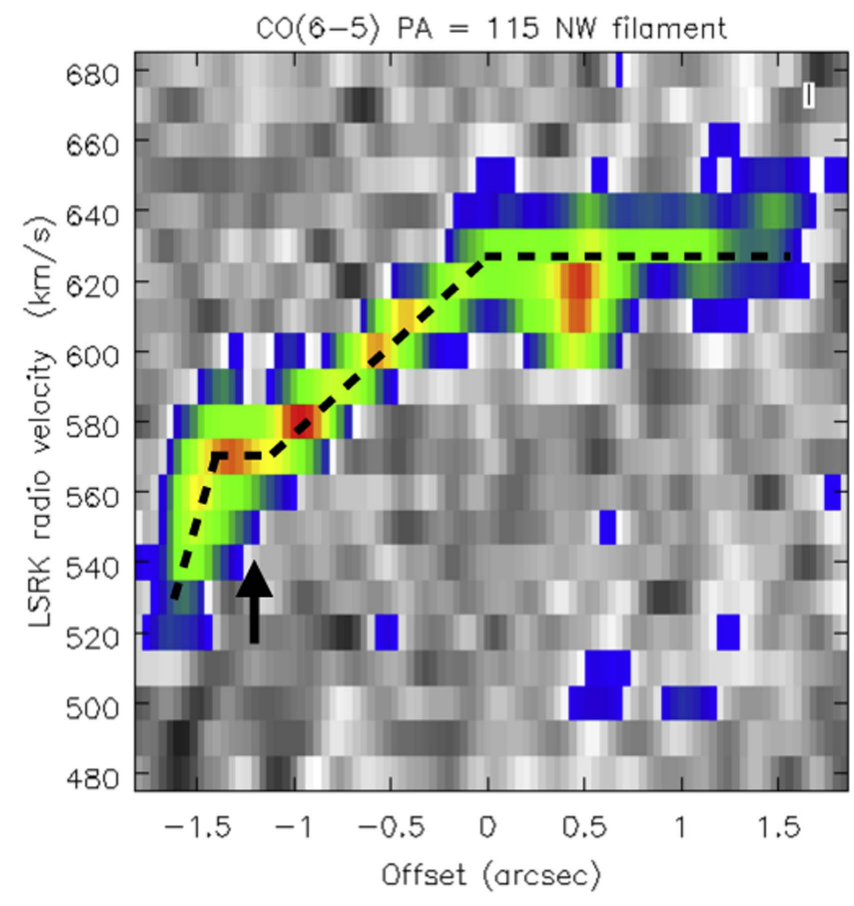

Figure 14. $\mathrm{CO}(6-5) \mathrm{P}-\mathrm{V}$ diagram at P.A. $=115^{\circ}$ along the $\mathrm{NW}$ filament with a length of $3 ! .7$ and a width of $1 / .9$, as in Figure 13(b) but with the velocity axis from 480 to $680 \mathrm{~km} \mathrm{~s}^{-1}$. The dashed lines show the velocity plateau and large velocity gradients along the filament. The (black) arrow symbol indicates the closest location to the AGN $(\sim 1$ !! 2 , or $22 \mathrm{pc})$ and systemic velocity.

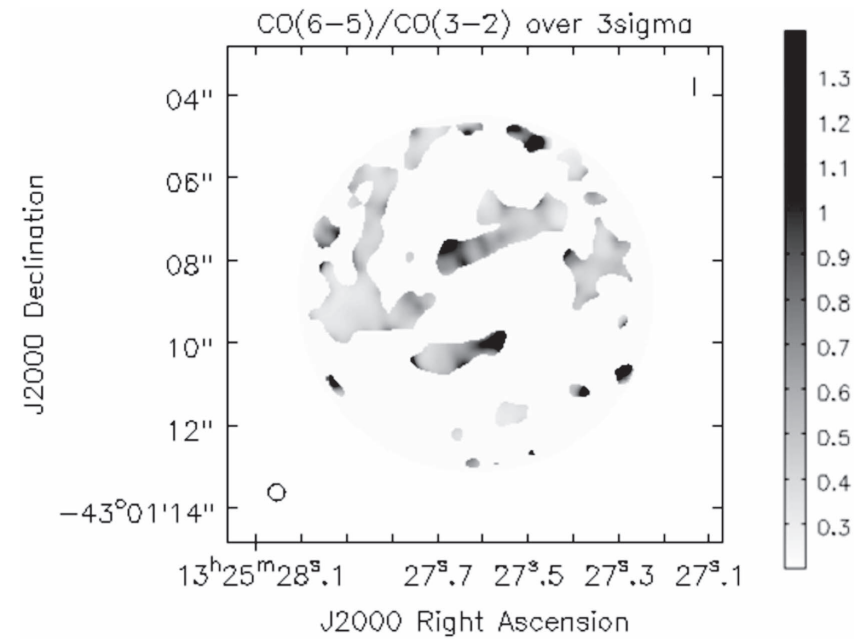

Figure 15. $\mathrm{CO}(6-5) / \mathrm{CO}(3-2)$ line ratio for values of the $\mathrm{CO}(6-5)$ and $\mathrm{CO}(3-2)$ maps exceeding $3 \sigma$, with an angular resolution of 0.4 (see bottomleft corner). The gray scale spans from 0.2 to 1.4 in $T_{\mathrm{mb}}$ units (or 0.9 to 5.9 in flux units).

gravitational constant. At $r=200 \mathrm{pc}$, and assuming an average velocity of $100 \mathrm{~km} \mathrm{~s}^{-1}$ and an inclination of the CND of $60^{\circ}$, $M_{\text {dyn }}=6 \times 10^{8} M_{\odot}$. Therefore, $M_{\text {gas }} / M_{\text {dyn }} \simeq 0.14$, which indicates that the CND is currently not an entity dominated by self-gravitation. Although this structure may have been generated as a result of a spontaneous gaseous bar, it is currently not selfgravitating.

In the case of Cen A, neither large-scale nor nuclear bars have been reported. The task of identifying such structures is complicated due to the large extinction toward the dust lane along the minor axis of this elliptical galaxy. The stellar component of Cen A appears very round, but it is likely that it is intrinsically flattened. Wilkinson et al. (1986) and Hui et al. (1995) support this scenario and find axis ratios of 1:0.98:0.55 and 1:0.92:0.79, respectively. The stellar component shows little rotation within one effective radius, with a maximum rotation of around $40 \mathrm{~km} \mathrm{~s}^{-1}$ roughly along the direction of the major axis at P.A.kin $\simeq 35^{\circ}$ (almost perpendicular to the dust lane) out to $100^{\prime \prime}$ from the nucleus (the $\mathrm{N}$ side is approaching and the S receding; Wilkinson et al. 1986). Silge et al. (2005) found that the stellar rotation is slower, $20 \mathrm{~km} \mathrm{~s}^{-1}$, at smaller radii of about $40^{\prime \prime}$. The velocity dispersion is $\sim 135 \mathrm{~km} \mathrm{~s}^{-1}$ on both axes for most of the radius range from $2^{\prime \prime}$ to $4^{\prime \prime}$ (Silge et al. 2005).

There is also an S-shape twist in the (stellar and planetary nebula) kinematics (Hui et al. 1995; Peng et al. 2004). Rotation at smaller scales of tens of parsec is actually P.A.kin $\simeq 165^{\circ}$, similar to that of the CND (i.e., $\sim 155^{\circ}$ ), but the nuclear stellar rotation is counterrotating relative to the gas (Cappellari et al. 2009).

Counterrotation between stars and gas might be an important aspect to explain the distribution and kinematics. The gas that was likely accreted from an H I-rich dwarf companion galaxy was probably not rotationally supported and then it went into orbits at a smaller distance from the center in a few orbital timescales (Morganti 2010). From the obtained circular velocities within the CND, $V_{c} \sim 200 \mathrm{~km} \mathrm{~s}^{-1}$. Therefore at the nuclear ring $(r=4 ! .5$, or $80 \mathrm{pc})$, the characteristic orbital period is $T \sim 1 \mathrm{Myr}$. The very short orbital period in the central regions suggests that although the gas should have already reached an equilibrium configuration, there are apparent asymmetries probably as a result of gas having recently reached the central region or being misaligned with respect to the gravitational potential. In the external regions farther out, the characteristic orbital period is larger, being $\sim 2 \times 10^{7}$ years at a radius of $1 \mathrm{kpc}$. Numerical simulations of the evolution of counterrotating gaseous disks in disk galaxies is such that there is a transitory $(\sim 500 \mathrm{Myr})$ main $m=1$ dynamical instability mode consisting of one-arm spirals which would be on the leading side with respect to the most massive disk, then transform into a more stationary phase with a composite of $m=1$ and $m=2$ features trailing and forming a ring (e.g., García-Burillo et al. 2000).

Another piece of information is that the nuclear filaments (i.e., loci of shocks) are straight and parallel to each other, although curved at large radii. From the inclination of the CND, the loci of shocks would be on the leading side of the putative nuclear bar, if any. From the morphology of the loci of shocks, one can infer the properties of the non-axisymmetric potential (e.g., Athanassoula 1992). Strong bars tend to have straight loci of shocks, while ovals or weak bars should have more curved shapes. Therefore, we can infer that somehow the gravitational potential would likely resemble that of a strong stellar bar.

Detailed hydrodynamical simulations would be needed to try to reproduce the molecular gas properties under such a complex stellar configuration and are beyond the scope of this paper. No matter what the origin is, the existence of the nuclear ring and shock loci leads to $m=2$ instabilities, which may play a major role in feeding AGNs of powerful radio sources.

\section{Geometry of the CND and Orientation of the Jet}

In this section, we revisit the question of whether the CND is perpendicular to the jet, which is expected if there is a physical 


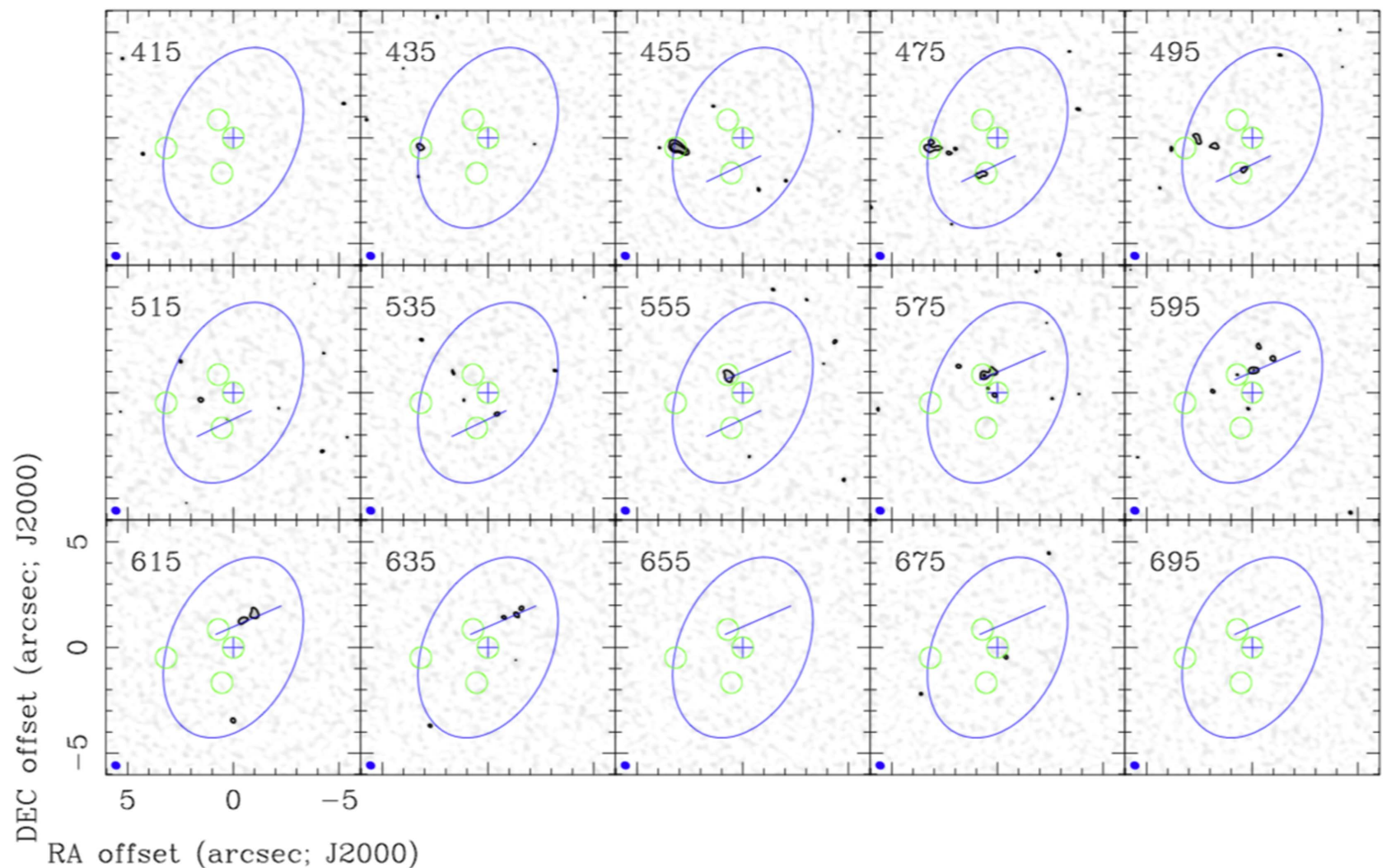

Figure 16. $\mathrm{HCO}^{+}(4-3)$ channel maps of the CND of Cen A in the LSR velocity range $V=415-695 \mathrm{~km} \mathrm{~s}^{-1}$ in $20 \mathrm{~km} \mathrm{~s}^{-1}$ bins. The size of the maps is $12^{\prime \prime}$. The velocities are shown in the upper-left corner and the synthesized beam at the bottom-left corner of each panel. The rms noise of individual channels is $1.3 \mathrm{mJy}$ beam ${ }^{-1}$. The contour levels are $3.5 \sigma$ and $7 \sigma$. The cross sign shows the position of the AGN: R.A. $=13^{\mathrm{h}} 25^{\mathrm{m}} 27^{\mathrm{s}} .615$, decl. $=-43^{\circ} 01^{\prime} 08^{\prime \prime} .805$. The $($ green) circles show the regions used to calculate the $\mathrm{HCO}^{+} / \mathrm{HCN}(4-3)$ ratio (Section 3.5) and spectra are presented in Figure 18. See Figure 4 for a description of the symbols representing the main molecular components of the CND of Cen A.

connection between the two. The relativistic jet is at a P.A. $\simeq 51^{\circ}$ and pointing toward us (e.g., Tingay et al. 1998), which is nearly perpendicular, at least in projection, to the CND, P.A. $\simeq 155^{\circ}$.

Here, we derived an inclination for the CND and nuclear ring of $i \simeq 60^{\circ}$ (near side is to the $S$ ) and $i \simeq 50^{\circ}$, respectively (see Section 3.6). These measurements are improvements over the inclination of $\simeq 70^{\circ}$ provided by Espada et al. (2009). The CND extent is $20^{\prime \prime}$, while that of the nuclear ring is half of that, so within $80<r<200 \mathrm{pc}$ it seems likely that the gas is nearly coplanar.

The inclinations are compatible with the smaller scale $(<54 \mathrm{pc})$ estimates by Neumayer et al. (2007) using VLT/SINFONI molecular hydrogen line data. The inclinations of the tilted rings in their warped disk model range from $i=38^{\circ}$ at $2 \mathrm{pc}$ to $i=59^{\circ}$ at $r>34$ pc. However, given that non-circular motions might be playing a major role as in the field of view probed by our ALMA observations (see Section 4), it is not clear how justified it is to model the molecular kinematics just using a pure warped disk model. In that case, maybe the average inclination of all of the concentric rings, $i=45^{\circ}$, would be a better estimate for the inclination of the nuclear disk. Finally, at an even smaller scale of $7-15$ mas $(0.13-0.25 \mathrm{pc})$ and at $12.5 \mu \mathrm{m}$, Burtscher et al. (2010), using the MID-infrared Interferometric instrument at the Very Large Telescope Interferometer, provided an orientation of dust emission with a P.A. of $\sim 10^{\circ}$, and an inclination of $\sim 63^{\circ}$, which overall does not seem compatible with the other measurements and may reflect more chaotic distribution at these small scales.
As for the jet, various estimates of its orientation have been published so far. Tingay et al. (1998), using VLBI observations, found a range for the axis of the nuclear jet with respect to the line of sight of $\theta \simeq 50^{\circ}-80^{\circ}$. Other estimates in the literature typically range from $\theta=60^{\circ}$ to $70^{\circ}$ (Dufour et al. 1979; Graham 1979; Skibo et al. 1994; Jones et al. 1996). These are in agreement with the inclinations that we find for the CND. VLBI observations by Müller et al. (2014) recently constrained the angle to the line of sight using the jet-tocounterjet brightness ratio, in combination with proper motions, to $\theta \sim 12^{\circ}-45^{\circ}$. A value close to $\sim 50^{\circ}$ would satisfy most constraints for the nuclear jet, and in that case the jet would be perpendicular to the circumnuclear and nuclear disks. The larger-scale (hundred parsec) radio jet using VLA seems to be compatible with smaller angles $\left(\sim 15^{\circ}\right)$, in contrast with the parsec-scale jet (Hardcastle et al. 2003).

Nuclear radio jets are seen to align well with the rotation axis of megamaser disks, even though the subparsec-scale accretion disks do not align with the kiloparsec-scale galactic disks (e.g., Greene et al. 2013). Possible causes for these misalignments are, among others, radiation pressure (e.g., Pringle 1997) and accretion events (e.g., Hopkins \& Quataert 2010). Therefore, a similar misalignment could also be present in Cen A, i.e., a warp of a few tens of degrees. In such a case, Nixon et al. (2012) find that disk misalignments boost accretion rate and angular momentum is dissipated in the interface where the two disks meet. 


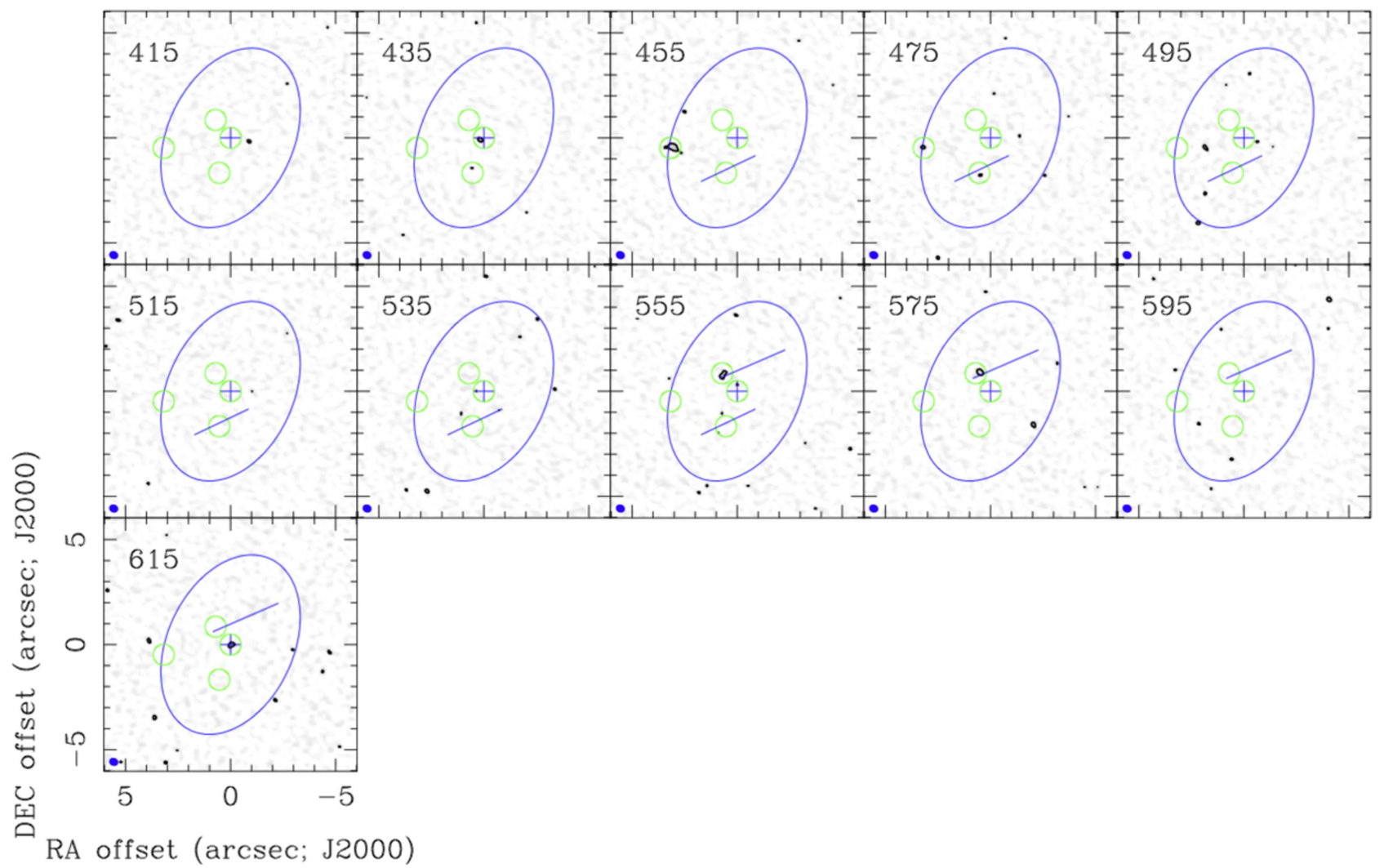

Figure 17. HCN(4-3) channel maps of the CND of Cen A in the LSR velocity range $V=415-615 \mathrm{~km} \mathrm{~s}^{-1}$ in $20 \mathrm{~km} \mathrm{~s}^{-1}$ bins. The size of the maps is $12^{\prime \prime}$. The velocities are shown in the upper-left corner and the synthesized beam at the bottom-left corner of each panel. The rms noise of individual channels is $1.3 \mathrm{mJy}$ beam ${ }^{-1}$. The contour levels are $3.5 \sigma$ and $7 \sigma$. The cross sign shows the position of the AGN: R.A. $=13^{\mathrm{h}} 25^{\mathrm{m}} 27^{\mathrm{s}} .615$, decl. $=-43^{\circ} 01^{\prime} 08^{\prime \prime} .805$. The (green) circles show the regions used to calculate the $\mathrm{HCO}^{+} / \mathrm{HCN}(4-3)$ ratio (Section 3.5) and spectra are presented in Figure 18. See Figure 4 for a description of the symbols representing the main molecular components of the CND of Cen A.

\section{Comparison with Numerical Simulations of the Multiphase ISM around SMBHs}

In this section, we compare the properties of the molecular gas in Cen A derived from our observations with recent numerical simulations studying feedback and the onset of thick tori in the center of disk galaxies. Numerical simulations have shown that the (energy-driven) jet feedback may create a cavity mostly devoid of dense gas in the nuclear region which quenches star formation, although the final distribution of dense gas depends on the initial configuration (e.g., Wagner et al. 2013). While negative feedback is most effective if clouds are tenuous and distributed spherically, positive feedback is most effective if clouds are in a disk-like configuration.

The jet of Cen A is very well collimated, with an opening angle of less than $12^{\circ}$ on scales of $0.3 \mathrm{pc}$ (Müller et al. 2011). There is no clear evidence in our maps of positive feedback such as gas compressed by the jet or negative feedback, where outflows may take gas away from the nuclear region (e.g., Matsushita et al. 2007, 2015; Fabian 2012; King \& Pounds 2015). In the CO maps, we could not discern any large velocity component that would resemble an outflowing component. However, the outflow might be more extended and recovering the whole flux in the maps could be important. Recently, Israel et al. (2017) reported the signature of an outflow in this target as seen in [C I] and $\mathrm{CO}(4-3)$ using the single-dish telescope APEX, as well as [C II], [N II], and [N III] using Herschel/PACS, although angular resolution is limited. Probably some of the most direct evidence of interaction between jet and surrounding gas is given by the fact that other ionized tracers are distributed almost along the direction of the jet ( $\mathrm{Br} \gamma$, [Fe II], [Si IV], [O IV] $(25.9 \mu \mathrm{m}),[\mathrm{Ne} \mathrm{V}](24.3 \mu \mathrm{m})$; e.g., Neumayer et al. 2007; Quillen et al. 2008). However, from arguments related to the geometry and kinematics, Neumayer et al. (2007) noted that ionized emission such as [Si VI] must indicate an inflow rather than outflow, and it is interpreted as the backflow of gas along the side of the jet's cocoon. Also, although farther apart, Salomé et al. (2016) and Morganti et al. (2016) reported the detection of molecular gas $15 \mathrm{kpc}$ NE from Cen A's center (i.e., in the region of the outer filament), where there is an ongoing interaction between the radio jet and gas clouds.

Three-dimensional radiative hydrodynamical models of the circumnuclear gas in galaxies predict that a clumpy torus-like structure is naturally produced on scales of tens of parsec around an $\mathrm{SMBH}$, due to radiative pressure from the AGN resulting in vertical circulation of gas (or fountains; Wada 2012), supernova-driven turbulence (Wada et al. 2002, 2009; Schartmann et al. 2009), as well as due to dynamical arguments related to galactic inflows (Hopkins et al. 2012). As a result of the energy feedback from SNe and AGNs, the circumnuclear disks possess a (i) highly inhomogeneous internal density and temperature structure, (ii) filamentary structures such as nuclear spiral arms, and (iii) large-scale height in the outer regions at galactocentric radii of a few tens of parsec, resembling the shape of a torus and being characterized by a large internal turbulent motion. Wada et al. (2016) modeled the multiphase properties of the ISM around an SMBH of $\sim 10^{6} M_{\odot}$ including the effect of AGN, 

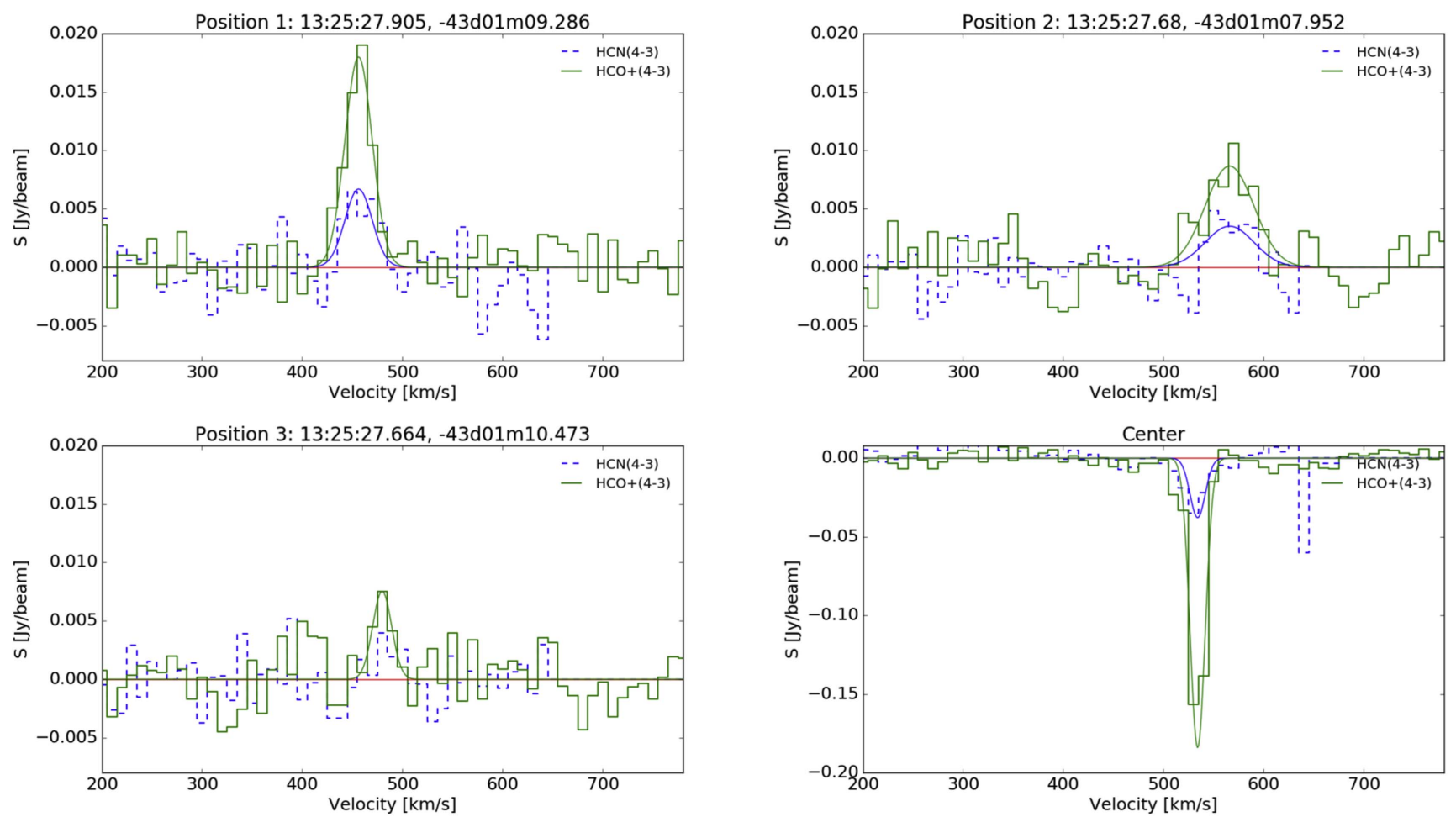

Figure 18. $\mathrm{HCN}$ and $\mathrm{HCO}^{+}(4-3)$ spectra (blue dashed and green solid lines, respectively) toward Position (1) R.A. $=13^{\mathrm{h}} 25^{\mathrm{m}} 27^{\mathrm{s}} .905$, decl. $=-43^{\circ} 01^{\prime} 09^{\prime \prime} .286$, Position (2) R.A. $=13^{\mathrm{h}} 25^{\mathrm{m}} 27^{\mathrm{s}} 68$, decl. $=-43^{\circ} 01^{\prime} 07^{\prime \prime}$ 952, Position (3) R.A. $=13^{\mathrm{h}} 25^{\mathrm{m}} 27^{\mathrm{s}} .664$, decl. $=-43^{\circ} 01^{\prime} 10^{\prime \prime}$ 473, and Position (4) center, at R.A. $=13^{\mathrm{h}} 25^{\mathrm{m}} 27^{\mathrm{s}} \cdot 615$, decl. $=-43^{\circ} 01^{\prime} 08^{\prime \prime} .805$ (see Figures 16 and 17). The $y$-axis of the spectra are shown in units of Jy/beam from -0.008 to 0.02 , except for the spectra toward the nucleus, which range from -0.2 to 0.008 , and the $x$-axis from 200 to $780 \mathrm{~km} \mathrm{~s}^{-1}$. Gaussian fits to the profiles are also shown, and line parameters are displayed in Table 3.

$\mathrm{SN}$, and XDR conditions. While the molecular hydrogen coexists at high $(T>1000 \mathrm{~K})$ and cold $(T<100 \mathrm{~K})$ temperatures, the dense and cold molecular ISM is expected to be distributed preferentially close to the equatorial plane.

Our observations agree in general with these numerical simulations. Our maps cover the central $400 \mathrm{pc}$ down to the $10 \mathrm{pc}$ scale. We observe a cold disk with filamentary and clumpy structure at scales of tens to hundreds of parsec. Several long filamentary structures are seen, and the general distribution is quite irregular and inhomogeneous. The velocity dispersion is larger at the edges of the CND, where multiple regions have velocity widths of $20 \mathrm{~km} \mathrm{~s}^{-1}$ up to $40 \mathrm{~km} \mathrm{~s}^{-1}$.

A measure of the molecular gas disk scale height can be estimated from the rotational velocity at a given radius, ranging roughly from $170 \mathrm{~km} \mathrm{~s}^{-1}$ in the center of the disk to $\sim 100 \mathrm{~km} \mathrm{~s}^{-1}$ at its edges (see Figure 20), and the velocity dispersion, which ranges from 10 to $40 \mathrm{~km} \mathrm{~s}^{-1}$, also from the center to its edges. We assume hydrostatic equilibrium of the molecular gas disk, an axisymmetric potential, and follow $h=R q \sigma / V$ (Equation (3.1) of Quillen et al. 1992), where $V$ is the velocity at radius $R, h$ is the scale height, and $q$ is the axis ratio of the potential, which is assumed to be $q \sim 1$. If $R=100-200 \mathrm{pc}, \sigma=10-40 \mathrm{~km} \mathrm{~s}^{-1}$ (although the larger velocity dispersion limit could be due to non-circular motions), and $V \simeq 200 \mathrm{~km} \mathrm{~s}^{-1}$, then $h=5-40 \mathrm{pc}$ from the inner to the outer radius. This increase in molecular gas scale height is not as large as in the scenario proposed by Wada et al. (2009) and Wada (2012), although it is unclear what the scale height should be for the different phases of the ISM for an object like Cen A, and in particular for the cold molecular gas.
There are other differences with respect to these numerical simulations. First, although filamentary and clumpy structures are present in the simulations, the loci of shocks and a ring structure are not well reproduced, which may reflect that a nonaxisymmetric potential (i.e., $m=2$ mode instability) would be needed in those simulations. Second, in the inner $20 \mathrm{pc}$, simulations predict a relatively cold and thin molecular disk to be formed, but such molecular gas component is not found as traced by $\mathrm{CO}(3-2)$ or $\mathrm{CO}(6-5)$ to our sensitivity limit. A warm $(T \sim 1000 \mathrm{~K})$ molecular disk is instead present in the inner tens of parsec.

\section{Physical and Chemical Properties of the Molecular Gas around the Cen $A$ Nucleus}

\subsection{CO Line Ratios}

Analysis of single-dish data for many $\mathrm{CO}$ transitions has shown that the CND of Cen A has an emission ladder of CO transitions quite different from those of either starburst galaxies or (Seyfert) AGNs (Israel et al. 2014).

While the average over the $20^{\prime \prime} \mathrm{CND}$ is $R_{65 / 32} \simeq 0.4$ using $T_{\mathrm{mb}}$ units (or 1.85 in flux units) (Israel et al. 2014), $\mathrm{CO}(6-5)$ to $\mathrm{CO}(3-2)$ line ratios in the inner half of the CND, as obtained from our ALMA data (Figure 15), are characterized by a larger average value, $R_{65 / 32}=0.9$ (or 3.5 in flux units). Nearby starforming galaxies, mostly classified as LIRGs, exhibit these high $R_{65 / 32}$ ratios globally, and even higher values are found in powerful radio galaxies such as 3C 293 (Papadopoulos et al. 2010).

The values that we find in the CND of Cen A are similar to those in the Seyfert 2 galaxy NGC 1068 at similar spatial 
Table 3

Gaussian Fits to $\mathrm{HCO}^{+}$and $\mathrm{HCN}(4-3)$ Spectra in the Brightest Detected Positions

\begin{tabular}{|c|c|c|c|c|c|c|}
\hline Position $^{\mathrm{a}}$ & $\begin{array}{l}\text { Distance } \\
\quad(\mathrm{pc})\end{array}$ & Line & $\begin{array}{c}\text { Flux } \\
\left(\mathrm{Jy} \mathrm{km} \mathrm{s}^{-1}\right)\end{array}$ & $\begin{array}{l}\text { Velocity } \\
\left(\mathrm{km} \mathrm{s}^{-1}\right)\end{array}$ & $\begin{array}{c}\text { Width } \\
\left(\mathrm{km} \mathrm{s}^{-1}\right)\end{array}$ & $\begin{array}{c}\text { Peak } \\
(\mathrm{Jy} / \text { beam })\end{array}$ \\
\hline \multirow[t]{2}{*}{1} & 58 & $\mathrm{HCO}^{+}(4-3)$ & $0.62 \pm 0.05$ & $461.2 \pm 1.3$ & $32 \pm 3$ & 0.018 \\
\hline & & $\mathrm{HCN}(4-3)$ & $0.23 \pm 0.04$ & 461.2 & 32.2 & 0.006 \\
\hline 2 & & $\mathrm{HCN}(4-3)$ & $0.21 \pm 0.04$ & 571 & 57 & 0.004 \\
\hline \multirow[t]{2}{*}{3} & 32 & $\mathrm{HCO}^{+}(4-3)$ & $0.18 \pm 0.05$ & $485 \pm 3$ & $22 \pm 7$ & 0.008 \\
\hline & & $\mathrm{HCN}(4-3)$ & $<0.1$ & & & \\
\hline
\end{tabular}

Note.

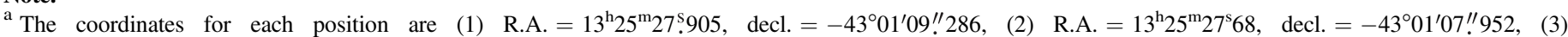
R.A. $=13^{\mathrm{h}} 25^{\mathrm{m}} 27^{\mathrm{s}} \cdot 664$, decl. $=-43^{\circ} 01^{\prime} 10^{\prime \prime} .473$, and Center: R.A. $=13^{\mathrm{h}} 25^{\mathrm{m}} 27^{\mathrm{s}} \cdot 615$, decl. $=-43^{\circ} 01^{\prime} 08^{\prime \prime} .805$.

scales. García-Burillo et al. (2014) show that the average $R_{65 / 32}$ ratio in the CND of NGC 1068 is $\sim 0.8$, with values ranging from $\sim 0.7$ to 2.0 (using $T_{\mathrm{mb}}$ units) close to the AGN. Aalto et al. (2017) also finds an average of 1.2 (also using $T_{\mathrm{mb}}$ units) in the nuclear regions of the lenticular galaxy NGC 1377, which is probably hosting a radio-quiet, obscured AGN (Costagliola et al. 2016).

In Section 3.4, we showed that there is a gradient of the $\mathrm{CO}(6-5)$ to $\mathrm{CO}(3-2)$ flux density ratios, $R_{65 / 32}$, along the nuclear filaments. There is gradual increase toward the AGN by a factor of 2-3, reaching the highest values closest to the nucleus (i.e., $\sim 20 \mathrm{pc}$ ), where maxima of $R_{65 / 32} \simeq 1.3$ using $T_{\mathrm{mb}}$ units (or 5 if flux units) are found. These high values should imply high density $\left(>10^{4} \mathrm{~cm}^{-3}\right)$ and high kinematic temperatures $\left(T_{\text {kin }}>100 \mathrm{~K}\right)$, similar to the centers of other galaxies observed on similar spatial scales (Matsushita et al. 1998, 2004; Hsieh et al. 2008; Izumi et al. 2013; García-Burillo et al. 2014; Aalto et al. 2017). Mechanical heating from shocks as well as illumination from X-ray and UV photons might be playing a role in the formation of such a gradient.

The higher ratio in the area probed by our ALMA observations with respect to the average in the CND suggests that $\mathrm{CO}$ emission will be more luminous with increasing transition preferentially toward the central regions.

\subsection{A Lack of $\mathrm{CO} / \mathrm{HCO}^{+} / \mathrm{HCN}$ Emission in the Inner $30 \mathrm{pc}$ ?}

An interesting difference in contrast with other galaxies also observed with matching spatial scales of $\sim 5 \mathrm{pc}$ is that there is not much molecular emission as traced by $\mathrm{CO}$ transitions in the center of Cen A. For example, NGC 1068 hosts in its center a nuclear torus (García-Burillo et al. 2016; Gallimore et al. 2016), while NGC 1377 shows a hot compact core dominated by gas along the jet (Aalto et al. 2017), which is extremely radio quiet in contrast to the one in Cen A. A question that remains open is whether this difference reflects different steps in the evolutionary sequence or just simply different scenarios and morphologies.

A caveat in the Cen A data is that toward the AGN we may find absorption lines. However, the continuum source is unresolved, so at least we would have expected to find emission from 10 to $30 \mathrm{pc}$ if the molecular distribution were centrally concentrated. The lack of detected emission is likely due to a large gradient in temperature and differences in the chemical properties of the ISM caused by the energetics from the AGN, because there exists molecular gas traced by $\mathrm{H}_{2}$
(Neumayer et al. 2007; see also Sections 1 and 3.6). In this very nuclear region, the molecules probed in this paper may have been mostly dissociated and ionized.

A consequence of this is that the molecular transitions such as those probed in this paper may not be good tracers of the molecular gas that obscures the AGN under the special conditions in the nuclear regions of objects like Cen $\mathrm{A}$, and that using $\mathrm{H}_{2}$ lines probing warmer gas might be more appropriate. This interpretation is in agreement with Hicks et al. (2009), who argued that the molecular gas as traced by the $\mathrm{H}_{2}$ line provides sufficient obscuring column densities toward a sample of local AGNs.

\section{3. $\mathrm{HCN} / \mathrm{HCO}^{+}$Line Ratios}

The dense gas tracers $\mathrm{HCN}$ and $\mathrm{HCO}^{+}$are detected in emission in the $J=4-3$ transitions toward several positions in the imaged region as well as in absorption toward the nucleus. Here we discuss the low $\mathrm{HCN} / \mathrm{HCO}^{+}$line ratios that are systematically found in the inner circumnuclear regions of Cen A, as mentioned in Section 3.5.

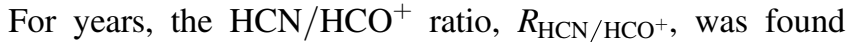
observationally to be $>1$ in the presence of an AGN and was claimed to be enhanced due to X-ray irradiation (e.g., Kohno et al. 2001; Krips et al. 2008). Theoretically, however, HCN and $\mathrm{HCO}^{+}$are generally of the same order in PDRs, but in $\mathrm{XDRs}, \mathrm{HCN}$ is not as bright as $\mathrm{HCO}^{+}$lines under the majority of conditions (Meijerink \& Spaans 2005). Three-dimensional radiative transfer simulations of $\mathrm{HCN}$ to $\mathrm{HCO}^{+}$line diagnostics in AGN molecular tori by Yamada et al. (2007) also show that under a wide range of molecular abundances $R_{\mathrm{HCN} / \mathrm{HCO}^{+}} \lesssim 1$.

Recently, the favored scenario for the enhancement of $\mathrm{HCN}$ around AGNs in observational data is high-temperature chemistry likely due to mechanical heating (Harada et al. 2010; Izumi et al. 2013; Matsushita et al. 2015). The increasing number of sources detected in these species seems to show on average an enhancement of the $\mathrm{HCN} / \mathrm{HCO}^{+}$ratio in the presence of an AGN (Privon et al. 2015; Imanishi et al. 2016; Izumi et al. 2016). However, the scatter is large among the ratios observed in both galaxies hosting AGNs and starbursts (Privon et al. 2015), which makes it necessary to study individual cases at high resolution to understand the origin of such a dispersion. In addition, recent high-resolution observations with ALMA have shown that this enhancement may occur in the surroundings and not toward the AGN itself 


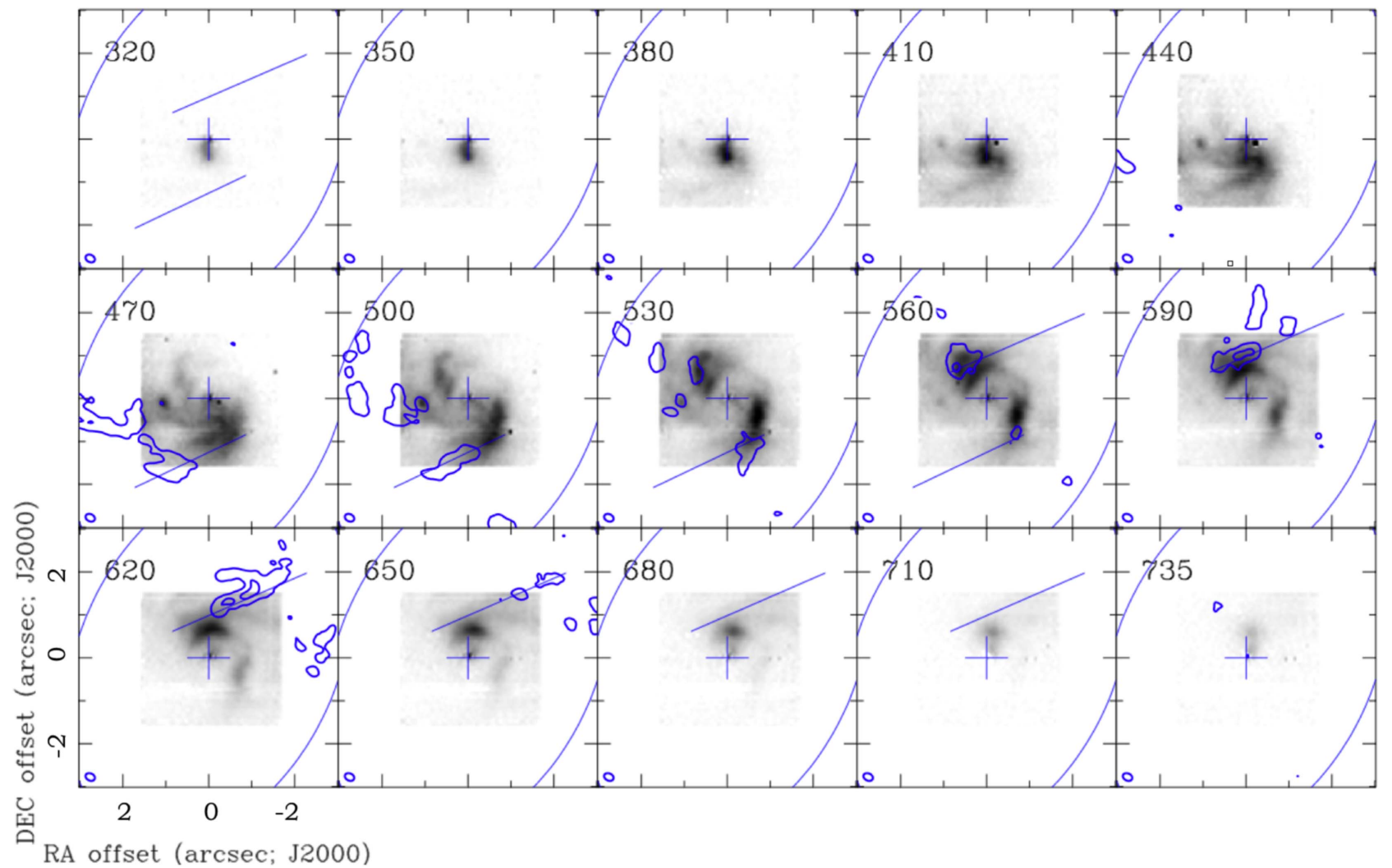

Figure 19. Comparison of the channel maps of the $\mathrm{H}_{2}(J=1-0) \mathrm{S}(1)$ (gray scale) and $\mathrm{CO}(6-5)$ (contours at $3.5 \sigma$ and $\left.7 \sigma\right)$ lines in the inner $6^{\prime \prime}$ of the $\mathrm{CND}$ of Cen $\mathrm{A}$ in the LSR velocity range $V=320-740 \mathrm{~km} \mathrm{~s}^{-1}$ in $30 \mathrm{~km} \mathrm{~s}^{-1}$ bins. The $\mathrm{H}_{2}$ map covers the inner $3^{\prime \prime}$ and the resolution of the $\mathrm{H}_{2}$ map is $0^{\prime \prime}$. 12 (Neumayer et al. 2007 ). The velocities are shown in the upper-left corner and the synthesized beam of the $\mathrm{CO}(6-5)$ observations at the bottom-left corner of each panel. The cross sign shows the position of the AGN: R.A. $=13^{\mathrm{h}} 25^{\mathrm{m}} 27^{\mathrm{s}} .615$, decl. $=-43^{\circ} 01^{\prime} 08^{\prime \prime} .805$. See Figure 4 for a description of the symbols representing the main molecular components of the CND of Cen A.

(García-Burillo et al. 2014; Martín et al. 2015; Izumi et al. 2016).

In regions where these dense tracers are detected in Cen A, all $R_{\mathrm{HCN} / \mathrm{HCO}^{+}}$ratios lie well below those of the AGNdominated galaxies (Imanishi et al. 2016) and within the range found for starburst galaxies (Izumi et al. 2016). Given our low $R_{\mathrm{HCN} / \mathrm{HCO}^{+}}$ratios, we can discard that conditions as in other AGN-dominated galaxies apply in the circumnuclear environments of Cen A. Given the shocked regions identified in this paper, possible interaction with the jet, and the elliptical nature of Cen A, one may expect that in these regions, mechanical heating is also important, and thus that high-temperature chemistry also apply, producing high $\mathrm{HCN}$ to $\mathrm{HCO}^{+}$ratios.

A possible explanation is that in Cen A, XDR conditions dominate over mechanical heating. The bolometric luminosity of the nuclear source of Cen A is estimated to be $2 \times 10^{43} \mathrm{erg} \mathrm{s}^{-1}$, half of it at high energies (Israel 1998). Detailed high angular resolution observations were obtained with Chandra, revealing nuclear emission, a jet, and more extended emission (Kraft et al. 2002). The $2-10 \mathrm{keV}$ unabsorbed luminosity of the nucleus of Cen $\mathrm{A}$ is estimated to be as high as $\sim 5 \times 10^{41} \mathrm{erg} \mathrm{s}^{-1}$ from Chandra and XMM-Newton, after correcting for the pile-up problem and adding extended emission (Evans et al. 2004). Fürst et al. (2016) use NuSTAR and XMM-Newton data and calculated an unabsorbed $3-50 \mathrm{keV}$ luminosity of $3.4 \times 10^{42} \mathrm{erg} \mathrm{s}^{-1}$. The source is not resolved (the point-spread function of $N u S T A R$ has a half-power diameter of $60^{\prime \prime}$ ), but it can be argued that the jet is mainly visible in the soft X-rays and most of the high energy radiation is coming from the nucleus itself. At even higher energies, in the $3-1000 \mathrm{keV}$ range, the luminosity is $L_{X}=$ $2.0 \times 10^{43} \mathrm{erg} \mathrm{s}^{-1}$ as seen by INTEGRAL (Beckmann et al. 2011). However, the X-ray luminosity of Cen A is in between other lowluminosity AGNs such as NGC 1097 (e.g., Nemmen et al. 2006; Izumi et al. 2013) and NGC 7469, and in these two cases the obtained $R_{\mathrm{HCN} / \mathrm{HCO}^{+}}$ratios toward their nuclei are $>1$ (Izumi et al. 2015). Cosmic-ray ionization rates might be higher in Cen $A$ producing even further an overdensity of $\mathrm{HCO}^{+}$over $\mathrm{HCN}$. Examples of low ratios can be found in M82 and NGC 1614, where enhanced cosmic-ray ionization rate due to frequent $\mathrm{SNe}$ would increase the abundance of $\mathrm{HCO}^{+}$even in a dense molecular cloud (e.g., Bayet et al. 2011; Meijerink et al. 2011; Aladro et al. 2013). However, it is likely that the number of SNe in Cen A is not that high. In the case of Cen A, the AGN itself may contribute (Aharonian et al. 2009). Another possible contribution to the low ratios is that $\mathrm{HCN}(4-3)$ is more subthermally excited than $\mathrm{HCO}^{+}(4-3)$ because of the difference in critical densities (Section 1). Also, as Cen A may have acquired the gas through an H I-rich dwarf galaxy, maybe the gas is less processed (i.e., it shows a lack of products of mostly secondary elements like N) and $\mathrm{HCN}$ would be underabundant with respect to $\mathrm{HCO}^{+}$.

Another variable to take into account is time. The $\mathrm{HCN} / \mathrm{HCO}^{+}$ abundance ratio is seen to be highly time dependent (Harada et al. 2013; García-Burillo et al. 2014), in the sense that although HCN might be overabundant in early times after the onset of chemical reactions, of the order of $10^{4-5}$ years $\left(t_{\text {cross }}\right.$, the shock crossing time), in steady state this might not be true. The age of AGN 


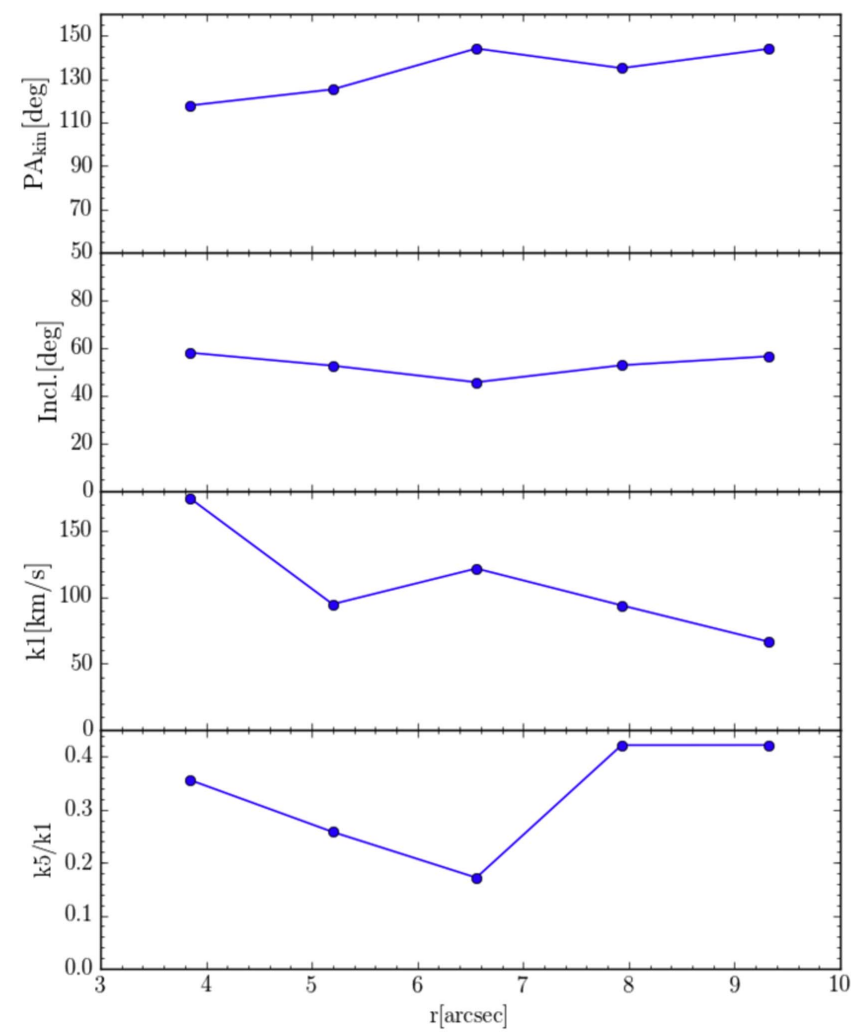

Figure 20. Kinemetry analysis (Krajnović et al. 2006) using the ALMA $\mathrm{CO}(3-2)$ velocity field with bins of 1 !'2: (a) the kinematic P.A., (b) the inclination (calculated from $q$, the axial ratio or flattening of the ellipse, assuming circular orbits), (c) $k 1$, the amplitude of bulk velocities, and (d) $k 5 / k 1$, the ratio between harmonics $k 1$ and $k 5$, where $k 5$ represents deviations from simple rotation. See Table 4 for details.

Table 4

Kinemetry Parameter Fits to the $\mathrm{CO}(3-2)$ Velocity Field

\begin{tabular}{lcccc}
\hline \hline $\begin{array}{l}\mathrm{r} \\
\prime \prime\end{array}$ & $\begin{array}{c}\text { Inclination } \\
(\mathrm{deg})\end{array}$ & $\begin{array}{c}\text { P.A. } \\
(\mathrm{deg})\end{array}$ & $\begin{array}{c}k_{1} \\
\left(\mathrm{~km} \mathrm{~s}^{-1}\right)\end{array}$ & $k_{1} / k_{5}$ \\
\hline 3.85 & 58 & 117 & 174 & 0.35 \\
5.19 & 53 & 125 & 94 & 0.25 \\
6.55 & 46 & 144 & 121 & 0.17 \\
7.93 & 52 & 135 & 90 & 0.42 \\
9.32 & 57 & 143 & 66 & 0.42 \\
\hline
\end{tabular}

Note. (a) The radial range considered, (b) the kinematic P.A., (b) $q$, the axial ratio or flattening of the ellipse, (c) $k 1$, the amplitude of bulk velocities, and (d) $k 5 / k 1$, the ratio between harmonics $k 1$ and $k 5$, where $k 5$ represents deviations from simple rotation.

activity in Cen $\mathrm{A}$ is considerably longer than that timescale, of the order of $10^{7-8}$ years (e.g., Morganti 2010 and references therein), so no overabundance of HCN would be expected then.

\section{Summary and Conclusions}

We have observed the molecular gas as traced by the $\mathrm{CO}(3-2), \mathrm{HCO}^{+}(4-3), \mathrm{HCN}(4-3)$, and $\mathrm{CO}(6-5)$ lines in the $\mathrm{CND}$ of the nearby elliptical and powerful radio galaxy Cen A. The angular resolution achieved using ALMA is $\sim 5 \mathrm{pc}(0 . ! 3)$. Our main findings are summarized as follows:

1. The CND's (unprojected) extent is $400 \mathrm{pc} \times 200 \mathrm{pc}$ along P.A. $=155^{\circ}$ and is composed of multiple filamentary
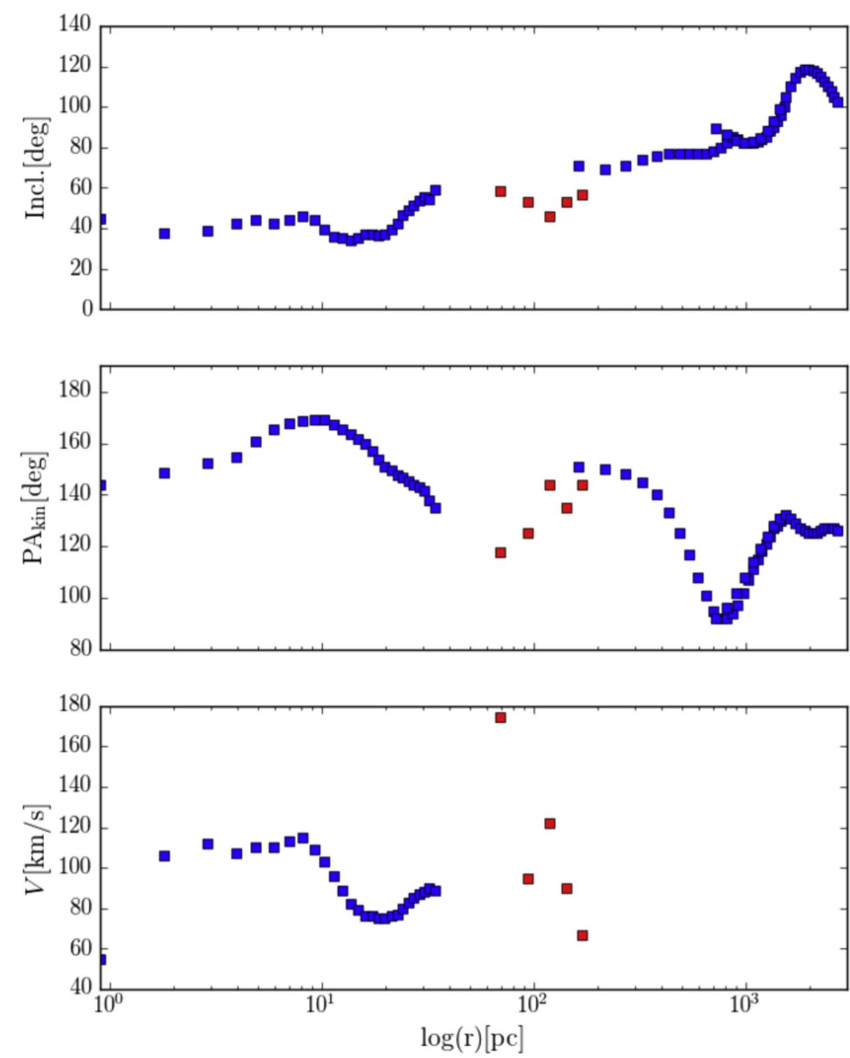

Figure 21. Inclination, P.A., and molecular gas bulk velocities (not corrected by inclination, projected rotational velocity if circular motions) as a function of radius from the $\mathrm{CO}(3-2)$ data presented in this paper (red square symbols) to be compared with the compilation carried out by Quillen et al. (2010 and references therein; blue square symbols). Note that rotational velocities are from Neumayer et al. (2007) and this paper. The compilation by Quillen et al. (2010) does not include velocities.

structures or streamers, which form a nuclear ring-like structure with an unprojected diameter of $9^{\prime \prime} \times 6^{\prime \prime}$ $(162 \mathrm{pc} \times 108 \mathrm{pc})$, and with a similar P.A. to that of the CND as a whole. The kinematic P.A. along the CND is slightly offset from $155^{\circ}$, ranging from $110^{\circ}$ to $150^{\circ}$.

2. Inside the nuclear ring-like feature, there are two nearly parallel filamentary structures with lengths of about $2^{\prime \prime}-3^{\prime \prime}$ (or $36-54 \mathrm{pc}$ ) at a P.A. $=120^{\circ}$, and with a rotational symmetry of $180^{\circ}$ with respect to each other and centered at the AGN. Although we confirm that $\mathrm{CO}$ emission is not bright in the inner $\sim 80 \mathrm{pc}$ inside the nuclear ring as previously suggested (Espada et al. 2009), we reveal for the first time that inside, there are nuclear filamentary structures. The line widths along these filamentary structures are as high as $\sim 100 \mathrm{~km} \mathrm{~s}^{-1}$, and are $\sim 50 \mathrm{~km} \mathrm{~s}^{-1}$ larger than those within the CND. We interpret these nuclear filaments to be shock regions caused by non-circular motions because of their distribution and distinct kinematics as revealed by the $\mathrm{P}-\mathrm{V}$ diagrams.

3. The velocity field within the CND presents an S-shape feature, indicative of non-circular motions and/or a warp in the disk. The CND, with a gradient of velocity from $315 \mathrm{~km} \mathrm{~s}^{-1}$ to $795 \mathrm{~km} \mathrm{~s}^{-1}$, can be separated using $\mathrm{P}-\mathrm{V}$ diagrams into three well-differentiated parts: (1) at $-8^{\prime \prime}$ and $8^{\prime \prime}$, there is a large velocity dispersion component of $\sim 100 \mathrm{~km} \mathrm{~s}^{-1}$, (2) $\Delta v / \Delta r=1.8 \mathrm{~km} \mathrm{~s}^{-1} \mathrm{pc}^{-1}$ in the inner parts of the CND, and (3) the nuclear filaments have a 

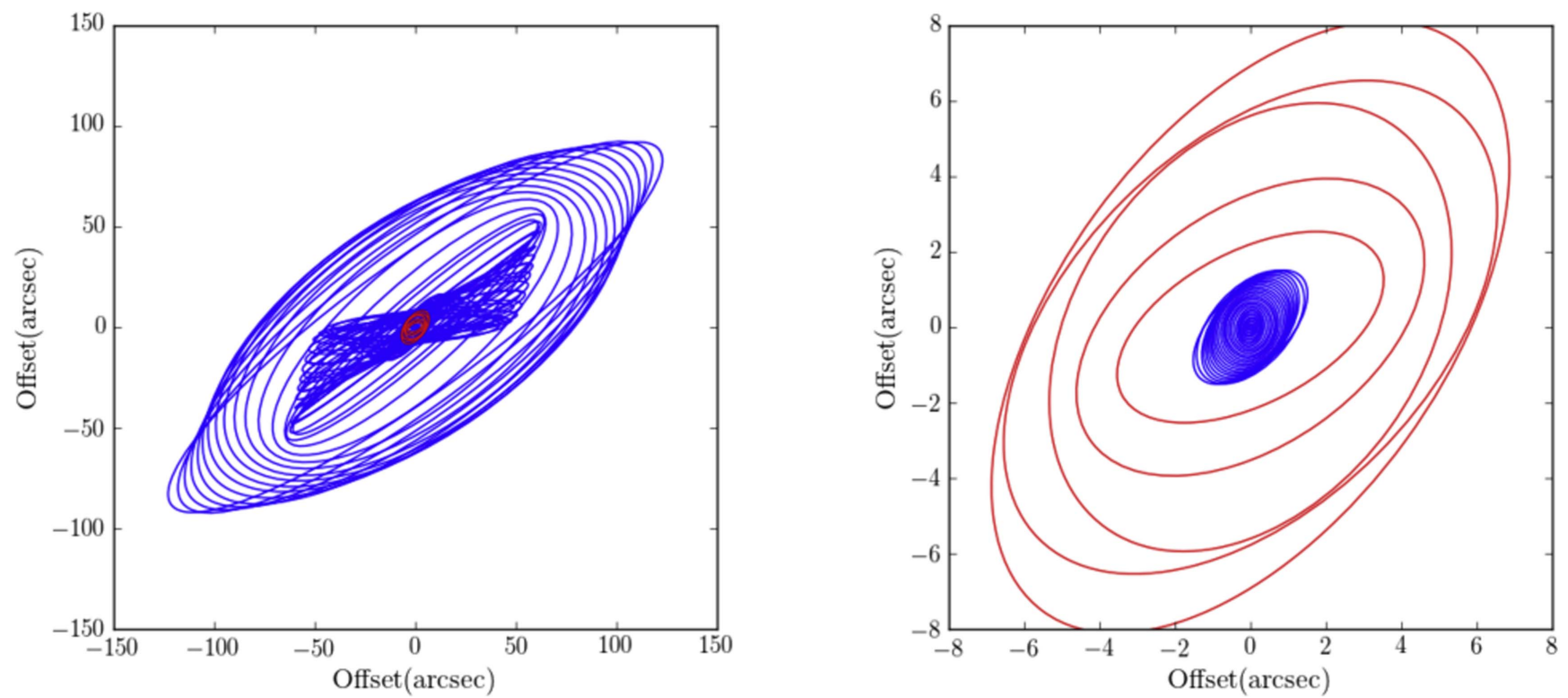

Figure 22. Ellipses (red) using the position and inclination angles as a function of radius fitted by Kinemetry using the $\mathrm{CO}(3-2)$ data, as presented in this paper (see Figure 20), and compared with other published fit results (blue ellipses): Quillen et al. (2006) in an inner region of 300" (left panel) and Neumayer et al. (2007) in an inner region of $16^{\prime \prime}$ (right panel).

distinct velocity gradient, even steeper, with $\Delta v / \Delta r=$ $3.4 \mathrm{~km} \mathrm{~s}^{-1} \mathrm{pc}^{-1}$.

4. Large velocity dispersions as high as $40 \mathrm{~km} \mathrm{~s}^{-1}$ are found toward the edges of the CND. If circular motions dominate at these radii and for hydrostatic equilibrium conditions, this can be interpreted as larger scale heights (up to $\sim 40 \mathrm{pc}$ ) as a function of radius. This increase of scale height is probably not as large as predicted by numerical simulations of the multiphase ISM of CNDs perturbed by $\mathrm{SNe}$ and $\mathrm{AGN}$ activities.

5. Low $\mathrm{HCN} / \mathrm{HCO}^{+}(4-3)$ ratios $(<0.5)$ are found toward several molecular clumps located at few tens of parsec from the AGN, unlike in the nuclear regions of other lowluminosity AGN galaxies, where high-temperature chemistry due to mechanical heating is invoked.

6. $\mathrm{CO}$ emission connects to the even warmer molecular gas probed using the $\mathrm{H}_{2}(J=1-0) \mathrm{S}(1)$ transition line within the inner $50 \mathrm{pc}$ (Neumayer et al. 2007). The gas winds up in the form of two or three main nuclear spirals as traced by the $\mathrm{H}_{2}$ line. There is no substantial emission in the molecular transitions we probed (i.e., $\mathrm{CO}(3-2), \mathrm{CO}(6-5)$, $\mathrm{HCN}$, and $\mathrm{HCO}^{+}(4-3)$ ) within the inner $20 \mathrm{pc}$ away from the AGN. A large gradient of excitation conditions must be present because molecular hydrogen emission in that region is prominent (Neumayer et al. 2007). Within $r \simeq 10 \mathrm{pc}, \mathrm{H}_{2}$ emission forms an inner ring structure and again another pair of filaments of $\sim 10 \mathrm{pc}$ length, with a rotational symmetry of $180^{\circ}$, along the $\mathrm{N}-\mathrm{S}$ direction, and with even larger velocity dispersions $\left(\sim 200 \mathrm{~km} \mathrm{~s}^{-1}\right)$ than anywhere else within the CND as probed in our CO maps, which may also indicate shocks at even smaller scales.

7. The overall molecular gas distribution and kinematics are interpreted as produced primarily by non-circular motions, similar to those in a bars within bars scenario, which are the successive shocked regions at different scales where loss of angular momentum occurs most efficiently. Although the disk might be warped, we argue that it is not the dominating factor. This study reveals observationally that non-circular motions and successive shocks are important mechanisms at play in feeding powerful radio galaxies down to parsec scales.

We thank the anonymous referee the comments and suggestions that have contributed to improve this paper. This paper makes use of the following ALMA data: ADS/JAO.ALMA\#2012.1.00225. S. ALMA is a partnership of ESO (representing its member states), NSF (USA) and NINS (Japan), together with NRC (Canada), NSC and ASIAA (Taiwan), and KASI (Republic of Korea), in cooperation with the Republic of Chile. The Joint ALMA Observatory is operated by ESO, AUI/NRAO, and NAOJ. We thank V. Impellizzeri and T. Wiklind for fruitful discussions. D.E. was supported by the ALMA Japan Research Grant of NAOJ Chile Observatory NAOJ-ALMA-0031. D.E. was supported by JSPS KAKENHI Grant Number JP 17K14254. S.M. is supported by the Ministry of Science and Technology (MoST) of Taiwan, MoST 103-2112-M-001-032-MY3. Data analysis was in part carried out on the open use data analysis computer system at the Astronomy Data Center, ADC, of the National Astronomical Observatory of Japan. This research made use of Astropy, a community-developed core Python package for Astronomy (Astropy Collaboration, 2013).

Facility: ALMA.

\section{References}

Aalto, S., Muller, S., Costagliola, F., et al. 2017, arXiv:1702.05458 Aharonian, F., Akhperjanian, A. G., Anton, G., et al. 2009, ApJL, 695, L40 Aladro, R., Viti, S., Bayet, E., et al. 2013, A\&A, 549, A39

Alard, C. 2001, A\&A, 379, L44

Allen, M. G., Sparks, W. B., Koekemoer, A., et al. 2002, ApJS, 139, 411 Astropy Collaboration, Robitaille, T. P., Tollerud, E. J., et al. 2013, A\&A, 558, A33 Athanassoula, E. 1992, MNRAS, 259, 345

Barth, A. J., Darling, J., Baker, A. J., et al. 2016, ApJ, 823, 51

Bayet, E., Williams, D. A., Hartquist, T. W., \& Viti, S. 2011, MNRAS, 414, 1583 Beckmann, V., Jean, P., Lubiński, P., Soldi, S., \& Terrier, R. 2011, A\&A, 531, A70 Bicknell, G. V., Sutherland, R. S., \& Neumayer, N. 2013, ApJ, 766, 36 Burtscher, L., Meisenheimer, K., Jaffe, W., Tristram, K. R. W., \& Röttgering, H. J. A. 2010, PASA, 27, 490

Cappellari, M., Neumayer, N., Reunanen, J., et al. 2009, MNRAS, 394, 660 
Combes, F. 1991, ARA\&A, 29, 195

Costagliola, F., Herrero-Illana, R., Lohfink, A., et al. 2016, A\&A, 594, A114

Cox, A. N. 2000, Allen's Astrophysical Quantities

Dufour, R. J., Harvel, C. A., Martins, D. M., et al. 1979, AJ, 84, 284

Eckart, A., Cameron, M., Genzel, R., et al. 1990, ApJ, 365, 522

Eckart, A., Wild, W., \& Ageorges, N. 1999, ApJ, 516, 769

Ekers, R. D., van Gorkom, J. H., Schwarz, U. J., \& Goss, W. M. 1983, A\&A, 122,143

Englmaier, P., \& Shlosman, I. 2004, ApJL, 617, L115

Erwin, P. 2004, A\&A, 415, 941

Espada, D. 2013, in ASP Conf. Ser. 476, New Trends in Radio Astronomy in the ALMA Era: The 30th Anniversary of Nobeyama Radio Observatory, ed. R. Kawabe, N. Kuno, \& S. Yamamoto (San Francisco, CA: ASP), 69

Espada, D., Matsushita, S., Peck, A., et al. 2009, ApJ, 695, 116

Espada, D., Matsushita, S., Peck, A. B., et al. 2012, ApJL, 756, L10

Espada, D., Peck, A. B., Matsushita, S., et al. 2010, ApJ, 720, 666

Evans, D. A., Kraft, R. P., Worrall, D. M., et al. 2004, ApJ, 612, 786

Fabian, A. C. 2012, ARA\&A, 50, 455

Fathi, K., Lundgren, A. A., Kohno, K., et al. 2013, ApJL, 770, L27

Fathi, K., Storchi-Bergmann, T., Riffel, R. A., et al. 2006, ApJL, 641, L25

Friedli, D. 1998, in IAU Symp. 184, The Central Regions of the Galaxy and Galaxies, ed. Y. Sofue (Dordrect: Kluwer), 269

Friedli, D., \& Martinet, L. 1993, A\&A, 277, 27

Fürst, F., Müller, C., Madsen, K. K., et al. 2016, ApJ, 819, 150

Gallimore, J. F., Elitzur, M., Maiolino, R., et al. 2016, ApJL, 829, L7

García-Burillo, S., Combes, F., Almeida, C. Ramos., et al. 2016, ApJL, 823, L12

García-Burillo, S., Combes, F., Usero, A., et al. 2014, A\&A, 567, A125

García-Burillo, S., Sempere, M. J., Combes, F., Hunt, L. K., \& Neri, R. 2000, A\&A, 363, 869

Graham, J. A. 1979, ApJ, 232, 60

Greene, J. E., Seth, A., den Brok, M., et al. 2013, ApJ, 771, 121

Harada, N., Herbst, E., \& Wakelam, V. 2010, ApJ, 721, 1570

Harada, N., Thompson, T. A., \& Herbst, E. 2013, ApJ, 765, 108

Hardcastle, M. J., Worrall, D. M., Kraft, R. P., et al. 2003, ApJ, 593, 169

Harris, G. L. H., Rejkuba, M., \& Harris, W. E. 2010, PASA, 27, 457

Hawarden, T. G., Sandell, G., Matthews, H. E., et al. 1993, MNRAS, 260, 844

Hicks, E. K. S., Davies, R. I., Malkan, M. A., et al. 2009, ApJ, 696, 448

Hopkins, P. F., Hayward, C. C., Narayanan, D., \& Hernquist, L. 2012, MNRAS, 420, 320

Hopkins, P. F., \& Quataert, E. 2010, MNRAS, 407, 1529

Hsieh, P.-Y., Matsushita, S., Lim, J., Kohno, K., \& Sawada-Satoh, S. 2008, ApJ, 683, 70

Hui, X., Ford, H. C., Freeman, K. C., \& Dopita, M. A. 1995, ApJ, 449, 592

Imanishi, M., Nakanishi, K., \& Izumi, T. 2016, AJ, 152, 218

Israel, F. P. 1998, A\&ARv, 8, 237

Israel, F. P., Güsten, R., Meijerink, R., et al. 2014, A\&A, 562, A96

Israel, F. P., Güsten, R., Meijerink, R., Requena-Torres, M. A., \& Stutzki, J. 2017, A\&A, 599, A53

Israel, F. P., van Dishoeck, E. F., Baas, F., et al. 1990, A\&A, 227, 342

Izumi, T., Kohno, K., Aalto, S., et al. 2015, ApJ, 811, 39

Izumi, T., Kohno, K., Aalto, S., et al. 2016, ApJ, 818, 42

Izumi, T., Kohno, K., Martín, S., et al. 2013, PASJ, 65, 100

Jones, D. L., Tingay, S. J., Murphy, D. W., et al. 1996, ApJL, 466, L63

Jungwiert, B., Combes, F., \& Axon, D. J. 1997, A\&AS, 125, 479

King, A., \& Pounds, K. 2015, ARA\&A, 53, 115

Kohno, K., Matsushita, S., Vila-Vilaró, B., et al. 2001, in ASP Conf. Ser. 249, The Central Kiloparsec of Starbursts and AGN: The La Palma Connection, ed. J. H. Knapen et al. (San Francisco, CA: ASP), 672

Kraft, R. P., Forman, W. R., Jones, C., et al. 2002, ApJ, 569, 54

Krajnović, D., Cappellari, M., de Zeeuw, P. T., \& Copin, Y. 2006, MNRAS, 366, 787

Krips, M., Neri, R., García-Burillo, S., et al. 2008, ApJ, 677, 262

Leeuw, L. L., Hawarden, T. G., Matthews, H. E., Robson, E. I., \& Eckart, A. 2002, ApJ, 565, 131

Liszt, H. 2001, A\&A, 371, 865

Lo, K. Y., \& Claussen, M. J. 1983, Natur, 306, 647

Ma, C., Arias, E. F., Eubanks, T. M., et al. 1998, AJ, 116, 516

Maloney, P., \& Black, J. H. 1988, ApJ, 325, 389

Marconi, A., Capetti, A., Axon, D. J., et al. 2001, ApJ, 549, 915

Marconi, A., Pastorini, G., Pacini, F., et al. 2006, A\&A, 448, 921

Martín, S., Kohno, K., Izumi, T., et al. 2015, A\&A, 573, A116

Martín, S., Martín-Pintado, J., Montero-Castaño, M., Ho, P. T. P., \& Blundell, R. 2012, A\&A, 539, A29

Matsushita, S., Kohno, K., Vila-Vilaro, B., Tosaki, T., \& Kawabe, R. 1998, ApJ, 495, 267
Matsushita, S., Muller, S., \& Lim, J. 2007, A\&A, 468, L49

Matsushita, S., Sakamoto, K., Kuo, C.-Y., et al. 2004, ApJL, 616, L55

Matsushita, S., Trung, D.-V., Boone, F., et al. 2015, ApJ, 799, 26

Mauersberger, R., Henkel, C., Wielebinski, R., Wiklind, T., \& Reuter, H.-P. 1996, A\&A, 305, 421

McMullin, J. P., Waters, B., Schiebel, D., Young, W., \& Golap, K. 2007, in ASP Conf. Ser. 376, Astronomical Data Analysis Software and Systems XVI, ed. R. A. Shaw, F. Hill, \& D. J. Bell (San Francisco, CA: ASP), 127 Meier, D. S., \& Turner, J. L. 2012, ApJ, 755, 104

Meier, D. S., Turner, J. L., \& Hurt, R. L. 2008, ApJ, 675, 281

Meijerink, R., \& Spaans, M. 2005, A\&A, 436, 397

Meijerink, R., Spaans, M., Loenen, A. F., \& van der Werf, P. P. 2011, A\&A, 525, A119

Mirabel, I. F., Laurent, O., Sanders, D. B., et al. 1999, A\&A, 341, 667

Morganti, R. 2010, PASA, 27, 463

Morganti, R., Oosterloo, T., Oonk, J. B. R., Santoro, F., \& Tadhunter, C. 2016, A\&A, 592, L9

Müller, C., Kadler, M., Ojha, R., et al. 2011, A\&A, 530, L11

Müller, C., Kadler, M., Ojha, R., et al. 2014, A\&A, 569, A115

Namekata, D., Habe, A., Matsui, H., \& Saitoh, T. R. 2009, ApJ, 691, 1525

Nemmen, R. S., Storchi-Bergmann, T., Yuan, F., et al. 2006, ApJ, 643, 652

Neumayer, N., Cappellari, M., Reunanen, J., et al. 2007, ApJ, 671, 1329

Nicholson, R. A., Bland-Hawthorn, J., \& Taylor, K. 1992, ApJ, 387, 503

Nixon, C. J., King, A. R., \& Price, D. J. 2012, MNRAS, 422, 2547

Ogle, P., Boulanger, F., Guillard, P., et al. 2010, ApJ, 724, 1193

Papadopoulos, P. P., van der Werf, P., Isaak, K., \& Xilouris, E. M. 2010, ApJ, 715,775

Peng, E. W., Ford, H. C., \& Freeman, K. C. 2004, ApJ, 602, 685

Pfenniger, D., \& Norman, C. 1990, ApJ, 363, 391

Phillips, T. G., Ellison, B. N., Keene, J. B., et al. 1987, ApJL, 322, L73

Pringle, J. E. 1997, MNRAS, 292, 136

Privon, G. C., Herrero-Illana, R., Evans, A. S., et al. 2015, ApJ, 814, 39

Quillen, A. C., Bland-Hawthorn, J., Green, J. D., et al. 2008, MNRAS, 384, 1469

Quillen, A. C., Brookes, M. H., Keene, J., et al. 2006, ApJ, 645, 1092

Quillen, A. C., de Zeeuw, P. T., Phinney, E. S., \& Phillips, T. G. 1992, ApJ, 391, 121

Quillen, A. C., Graham, J. R., \& Frogel, J. A. 1993, ApJ, 412, 550

Quillen, A. C., Neumayer, N., Oosterloo, T., \& Espada, D. 2010, PASA, 27, 396

Roberts, M. S. 1970, ApJL, 161, L9

Rydbeck, G., Wiklind, T., Cameron, M., et al. 1993, A\&A, 270, L13

Sakamoto, K., Okumura, S. K., Ishizuki, S., \& Scoville, N. Z. 1999, ApJS, 124,403

Salomé, Q., Salomé, P., Combes, F., \& Hamer, S. 2016, A\&A, 595, A65

Sarma, A. P., Troland, T. H., \& Rupen, M. P. 2002, ApJ, 564, 696

Sault, R. J., Teuben, P. J., \& Wright, M. C. H. 1995, in ASP Conf. Ser. 77, Astronomical Data Analysis Software and Systems IV, ed. R. A. Shaw, H. E. Payne, \& J. J. E. Hayes (San Francisco, CA: ASP), 433

Schartmann, M., Meisenheimer, K., Klahr, H., et al. 2009, MNRAS, 393, 759

Schinnerer, E., Böker, T., Emsellem, E., \& Downes, D. 2007, A\&A, 462, L27 Schinnerer, E., Böker, T., Emsellem, E., \& Lisenfeld, U. 2006, ApJ, 649, 181 Shlosman, I., Begelman, M. C., \& Frank, J. 1990, Natur, 345, 679 Shlosman, I., Frank, J., \& Begelman, M. C. 1989, Natur, 338, 45

Silge, J. D., Gebhardt, K., Bergmann, M., \& Richstone, D. 2005, AJ, 130, 406 Skibo, J. B., Dermer, C. D., \& Kinzer, R. L. 1994, ApJL, 426, L23

Struve, C., Oosterloo, T. A., Morganti, R., \& Saripalli, L. 2010, A\&A, 515, A67

Tingay, S. J., Jauncey, D. L., Reynolds, J. E., et al. 1998, AJ, 115, 960

Tsuboi, M., Kitamura, Y., Miyoshi, M., et al. 2016, PASJ, 68, L7 van der Hulst, J. M., Golisch, W. F., \& Haschick, A. D. 1983, ApJL, 264, L37 Wada, K. 2012, ApJ, 758, 66

Wada, K., Meurer, G., \& Norman, C. A. 2002, ApJ, 577, 197

Wada, K., Papadopoulos, P. P., \& Spaans, M. 2009, ApJ, 702, 63

Wada, K., Schartmann, M., \& Meijerink, R. 2016, ApJL, 828, L19

Wada, K., Taniguchi, Y., Habe, A., \& Hasegawa, T. 1994, ApJL, 437, L123

Wagner, A. Y., Umemura, M., \& Bicknell, G. V. 2013, ApJL, 763, L18

Weiß, A., Neininger, N., Hüttemeister, S., \& Klein, U. 2001, A\&A, 365, 571

Wiklind, T., \& Combes, F. 1997, A\&A, 324, 51

Wilkinson, A., Sharples, R. M., Fosbury, R. A. E., \& Wallace, P. T. 1986, MNRAS, 218, 297

Wilson, C. D. 1995, ApJL, 448, L97

Wozniak, H., Friedli, D., Martinet, L., Martin, P., \& Bratschi, P. 1995, A\&AS, 111,115

Yamada, M., Wada, K., \& Tomisaka, K. 2007, ApJ, 671, 73

Zhao, J.-H., Morris, M. R., Goss, W. M., \& An, T. 2009, ApJ, 699, 186 